Aus der Abteilung Nephrologie und Rheumatologie (Prof. Dr. med. G.A. Müller) im Zentrum Innere Medizin der Medizinischen Fakultät der Universität Göttingen

\title{
Die Bedeutung von Risikofaktoren für das
} Auftreten von Komplikationen nach Nierenbiopsie

INAUGURAL - DISSERTATION

zur Erlangung des Doktorgrades

der Medizinischen Fakultät

der Georg-August-Universität zu Göttingen

vorgelegt von

Sylvia Maria Wachendorfer

aus

Albstadt

Göttingen 2009 
Dekan: Prof. Dr. med. C. Frömmel

I. Berichterstatter: Prof. Dr. med. F. Strutz

II. Berichterstatter/in:

III. Berichterstatter/in:

Tag der mündlichen Prüfung: 


\section{Inhaltsverzeichnis}

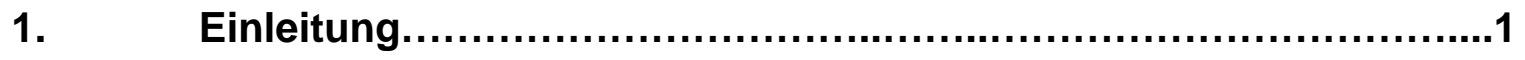

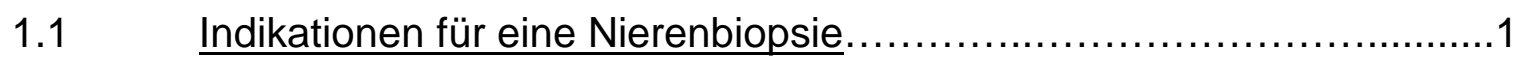

1.1.1 Akutes Nierenversagen.........................................................

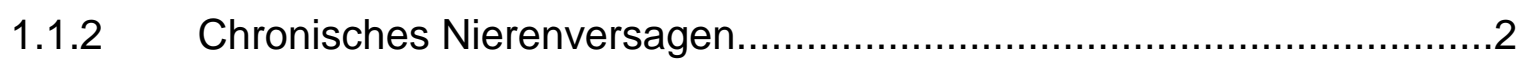

1.1.3 Asymptomatische Hämaturie......................................................... 3

1.1.4 Asymptomatische Proteinurie ohne Systemerkrankung........................3

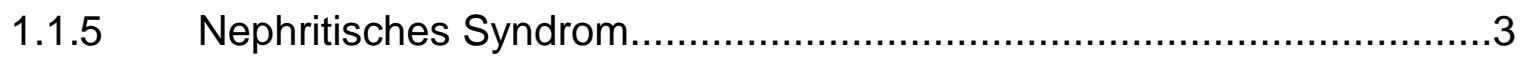

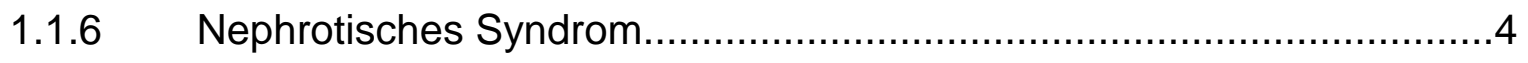

1.2 Durchführung einer Nierenbiopsie.........................................

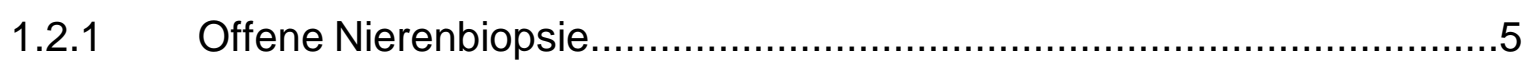

1.2.2 Laparoskopische Nierenbiopsie....................................................

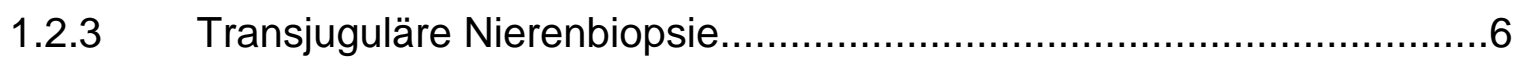

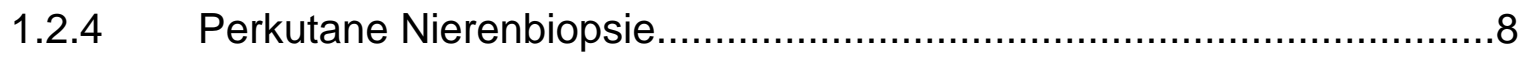

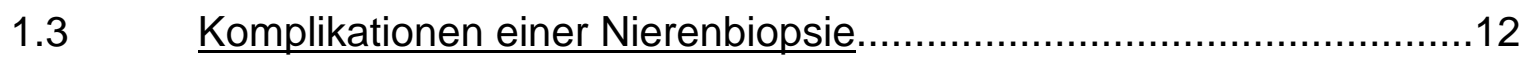

$1.4 \quad$ Platelet function analyzer (PFA)-100 TM ................................14

2. Patienten und Methoden..................................................17

2.1 Studiendesign und Patienten..............................................

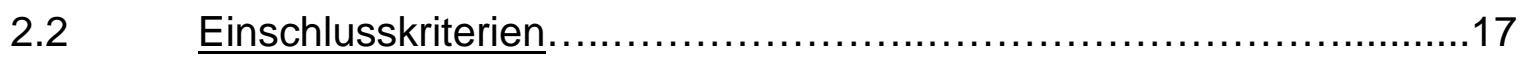

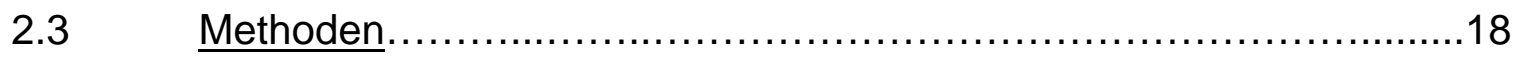

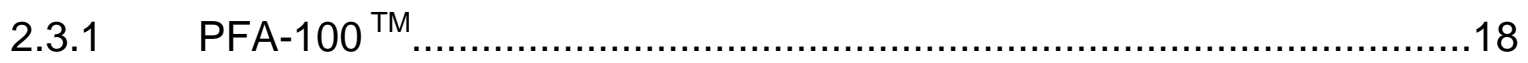

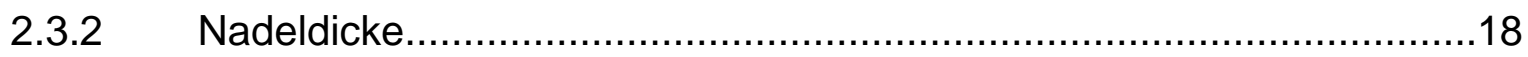

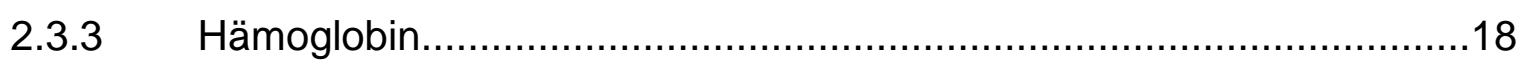

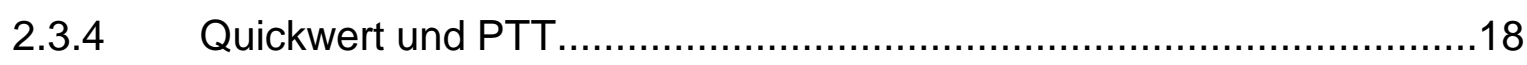

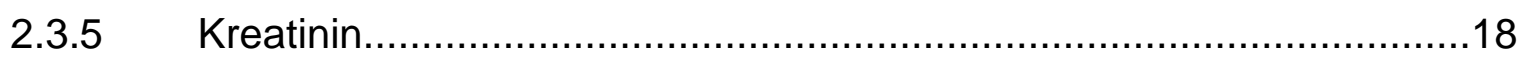

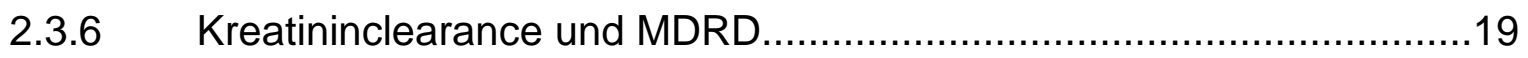

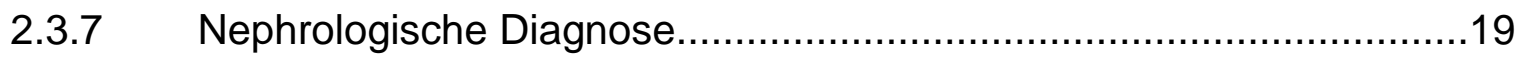

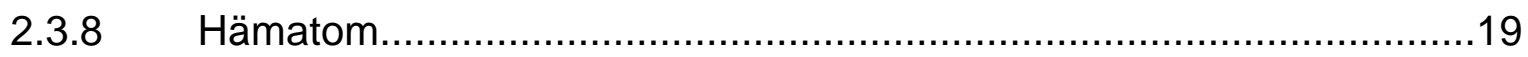

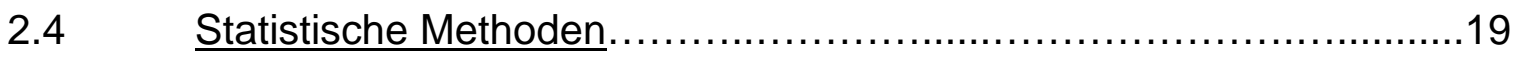




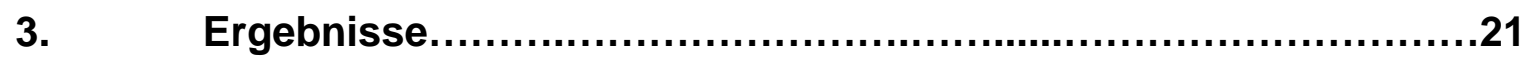

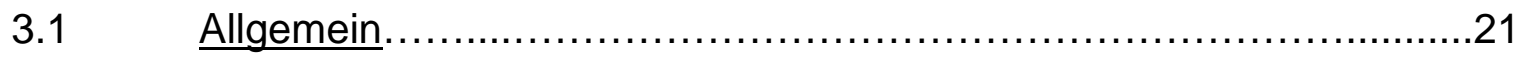

3.2 Übersicht über die statistischen Ergebnisse ...............................25

3.3 PFA-100 ${ }^{\mathrm{TM}}$ als Parameter zur Evaluation von Risiken........................34

3.3.1 Berechnung mit ordinalskalierten Werten...........................................34

3.3.1.1 Beziehung zwischen PFA-100 ${ }^{\mathrm{TM}}$ und Hämatom.................................34

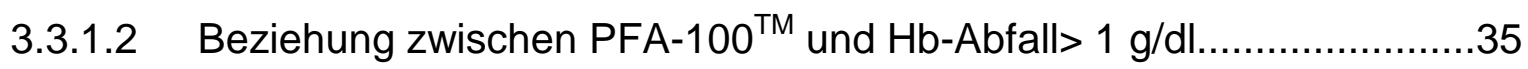

3.3.1.3 Beziehung zwischen PFA-100 ${ }^{\mathrm{TM}}$ und Komplikation.............................35

3.3.1.4 Beziehung zwischen PFA-100 ${ }^{\mathrm{TM}}$ und Komplikationsart.......................36

3.3.2 Berechnung mit nominalskalierten Werten.......................................38

3.3.2.1 Beziehung zwischen PFA-100 ${ }^{\mathrm{TM}}$ und Hämatom.................................39

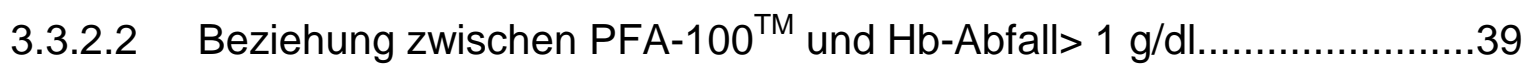

3.3.2.3 Beziehung zwischen PFA-100 ${ }^{\mathrm{TM}}$ und Komplikation............................40

3.3.2.4 Beziehung zwischen PFA-100 ${ }^{\mathrm{TM}}$ und Komplikationsart........................41

3.4 Das Geschlecht als Parameter zur Evaluation von Risiken...............42

3.4.1 Beziehung zwischen Geschlecht und Hämatom...............................42

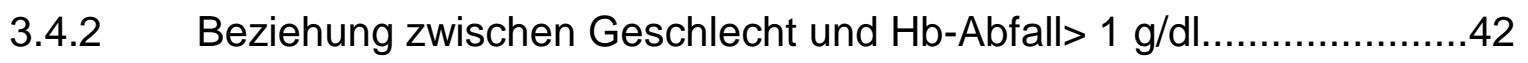

3.4.3 Beziehung zwischen Geschlecht und Komplikation...........................42

3.4.4 Beziehung zwischen Geschlecht und Komplikationsart......................42

3.5 Das Alter als Parameter zur Evaluation von Risiken.......................43

3.5.1 Beziehung zwischen Alter und Hämatom........................................43

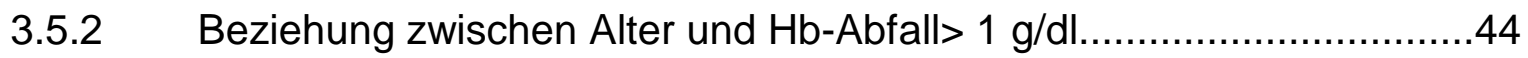

3.5.3 Beziehung zwischen Alter und Komplikation.....................................44

3.5.4 Beziehung zwischen Alter und Komplikationsart................................4

3.6 Die Nadeldicke als Parameter zur Evaluation von Risiken....................45

3.6.1 Beziehung zwischen Nadeldicke und Hämatom.................................45

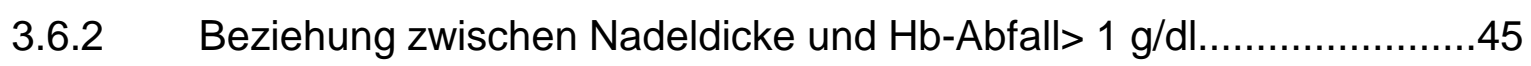

3.6.3 Beziehung zwischen Nadeldicke und Komplikation............................45

3.6.4 Beziehung zwischen Nadeldicke und Komplikationsart.......................45

3.7 Die Dauer des stationären Aufenthaltes als Parameter zur Evaluation von Risiken............................................46

3.7.1 Beziehung zwischen stationärem Aufenthalt und Hämatom.................46

3.7.2 Beziehung zwischen stationärem Aufenthalt und $\mathrm{Hb}$-Abfall> $1 \mathrm{~g} / \mathrm{dl} . . . .46$

3.7.3 Beziehung zwischen stationärem Aufenthalt und Komplikation............47 
3.7.4 Beziehung zwischen stationärem Aufenthalt und Komplikationsart.....47

3.8 Der Hämoglobingehalt $(\mathrm{Hb})$ als Parameter zur Evaluation von

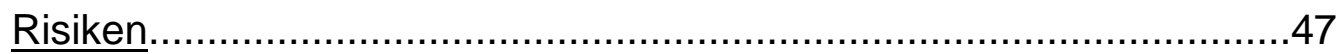

3.8.1 Beziehung zwischen $\mathrm{Hb}$ und Hämatom.......................................... 47

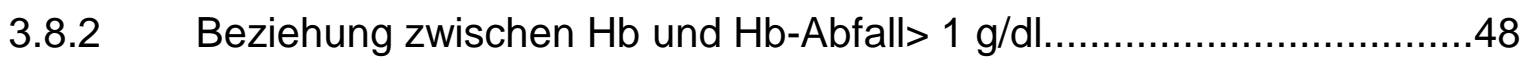

3.8.3 Beziehung zwischen $\mathrm{Hb}$ und Komplikation......................................48

3.8.4 Beziehung zwischen $\mathrm{Hb}$ und Komplikationsart.................................48

3.9 Der Quickwert als Parameter zur Evaluation von Risiken...................50

3.9.1 Berechnung mit ordinalskalierten Werten......................................50

3.9.1.1 Beziehung zwischen Quick und Hämatom........................................50

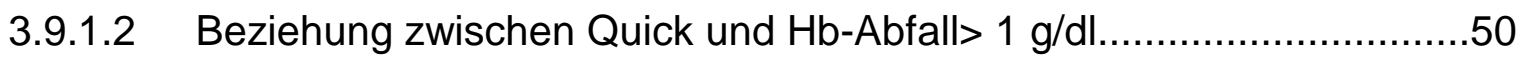

3.9.1.3 Beziehung zwischen Quick und Komplikation..................................51

3.9.1.4 Beziehung zwischen Quick und Komplikationsart..............................51

3.9.2 Berechnung mit nominalskalierten Werten.....................................51

3.9.2.1 Beziehung zwischen Quick und Hämatom.......................................51

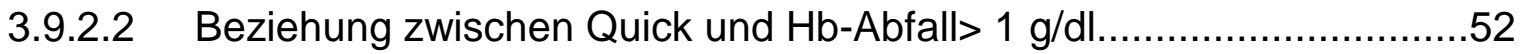

3.9.2.3 Beziehung zwischen Quick und Komplikation..................................52

3.9.2.4 Beziehung zwischen Quick und Komplikationsart..............................52

3.10 Die PTT als Parameter zur Evaluation von Risiken.......................53

3.10.1 Beziehung zwischen PTT und Hämatom.......................................53

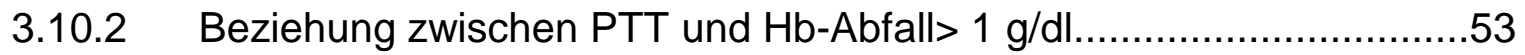

3.10.3 Beziehung zwischen PTT und Komplikation....................................53

3.10.4 Beziehung zwischen PTT und Komplikationsart...............................53

3.11 Kreatinin als Parameter zur Evaluation von Risiken......................54

3.11.1 Beziehung zwischen Kreatinin und Hämatom...................................55

3.11.2 Beziehung zwischen Kreatinin und Hb-Abfall> $1 \mathrm{~g} / \mathrm{dl}$.........................55

3.11.3 Beziehung zwischen Kreatinin und Komplikation..............................55

3.11.4 Beziehung zwischen Kreatinin und Komplikationsart..........................56

3.12 Die Kreatininclearance als Parameter zur Evaluation von Risiken......57

3.12.1 Beziehung zwischen Kreatininclearance und Hämatom.....................58

3.12.2 Beziehung zwischen Kreatininclearance und Hb-Abfall> $1 \mathrm{~g} / \mathrm{dl} \ldots \ldots \ldots . . .58$

3.12.3 Beziehung zwischen Kreatininclearance und Komplikation.................58

3.12.4 Beziehung zwischen Kreatininclearance und Komplikationsart...........59

3.13 Die MDRD - Formel als Parameter zur Evaluation von Risiken.........60 
3.13.1 Berechnung mit ordinalskalierten Werten.......................................60

3.13.1.1 Beziehung zwischen MDRD und Hämatom.....................................60

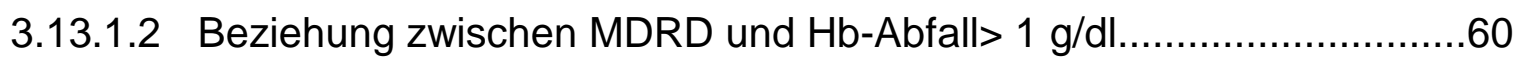

3.13.1.3 Beziehung zwischen MDRD und Komplikation.................................60

3.13.1.4 Beziehung zwischen MDRD und Komplikationsart............................61

3.13.2 Berechnung mit nominalskalierten Werten......................................62

3.13.2.1 Beziehung zwischen MDRD und Hämatom......................................63

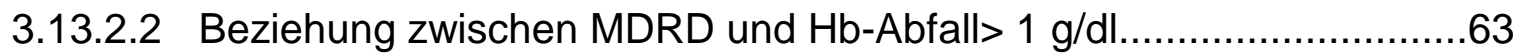

3.13.2.3 Beziehung zwischen MDRD und Komplikation.................................63

3.13.2.4 Beziehung zwischen MDRD und Komplikationsart.............................64

3.14 Sonstige Erkrankungen als Parameter zur Evaluation von Risiken.....64

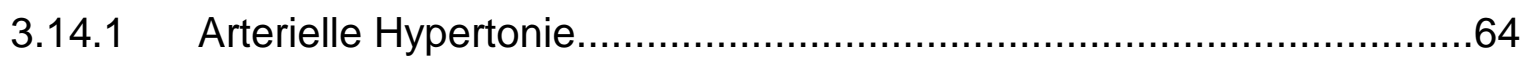

3.14.1.1 Beziehung zwischen arterieller Hypertonie und Hämatom...................64

3.14.1.2 Beziehung zwischen arterieller Hypertonie und Hb-Abfall> $1 \mathrm{~g} / \mathrm{dl} . . . \ldots . . .65$

3.14.1.3 Beziehung zwischen arterieller Hypertonie und Komplikation...............65

3.14.1.4 Beziehung zwischen arterieller Hypertonie und Komplikationsart........65

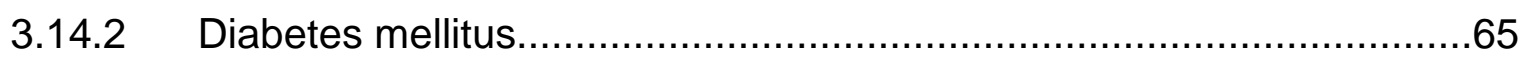

3.14.2.1 Beziehung zwischen Diabetes mellitus und Hämatom........................65

3.14.2.2 Beziehung zwischen Diabetes mellitus und $\mathrm{Hb}$-Abfall> $1 \mathrm{~g} / \mathrm{dl} \ldots \ldots \ldots \ldots \ldots . . .66$

3.14.2.3 Beziehung zwischen Diabetes mellitus und Komplikation....................66

3.14.2.4 Beziehung zwischen Diabetes mellitus und Komplikationsart..............66

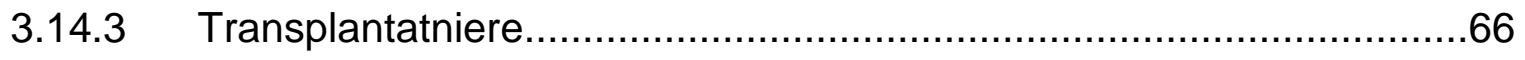

3.14.3.1 Beziehung zwischen Transplantatniere und Hämatom.......................66

3.14.3.2 Beziehung zwischen Transplantatniere und Hb-Abfall> $1 \mathrm{~g} / \mathrm{dl} . \ldots \ldots \ldots . . . . .67$

3.14.3.3 Beziehung zwischen Transplantatniere und Komplikation...................67

3.14.3.4 Beziehung zwischen Transplantatniere und Komplikationsart.............67

3.15 Die nephrologische Diagnose als Parameter

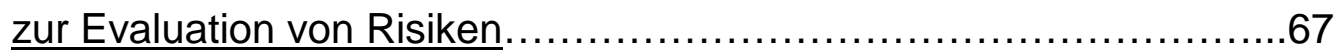

3.15.1 Beziehung zwischen nephrologischer Diagnose und Hämatom...........67

3.15.2 Beziehung zwischen nephrologischer Diagnose und

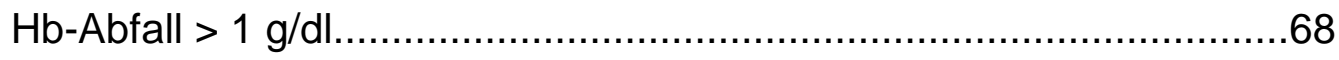

3.15.3 Beziehung zwischen nephrologischer Diagnose und Komplikation.....68

3.15.4 Beziehung zwischen nephrologischer Diagnose und Komplikationsart 
3.16 Komplikationsrate bei Eigenniere und nach Nierentransplantation......69

3.16.1 Komplikationsrate bei Eigenniere.................................................69

3.16.2 Komplikationsrate nach Nierentransplantation..................................71

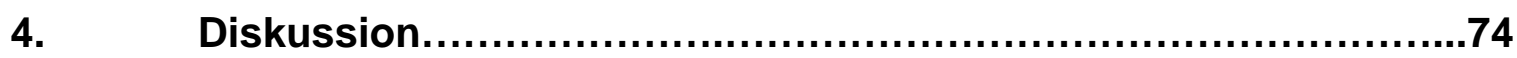

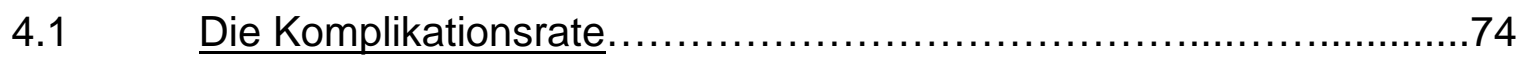

4.2 PFA-100 ${ }^{\mathrm{TM}}$ als Risikofaktor für Komplikationen............................75

4.3 Das Geschlecht als Risikofaktor für Komplikationen.......................77

4.4 Das Alter als Risikofaktor für Komplikationen............................78

4.5 Die Nadeldicke als Risikofaktor für Komplikationen.......................78

4.6 Beobachtungszeitraum und Dauer des stationären Aufenthaltes.......80

4.7 Der $\mathrm{Hb}$ - Wert als Risikofaktor für Komplikationen........................81

4.8 Die Blutgerinnung als Risikofaktor für Komplikationen...................82

4.9 Die Nierenfunktion als Risikofaktor für Komplikationen....................83

4.10 Begleiterkrankungen als Risikofaktor für Komplikationen..................86

4.11 Die nephrologische Diagnose als Risikofaktor für Komplikationen......88

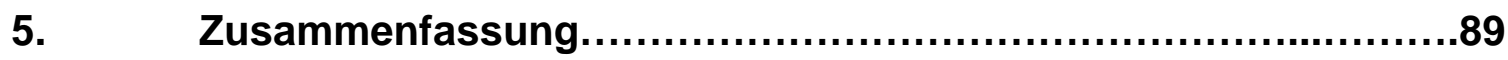

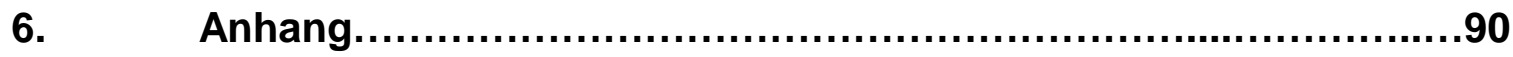

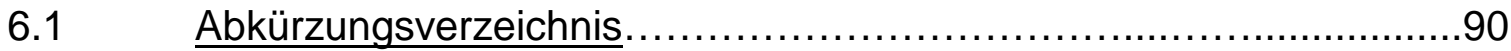

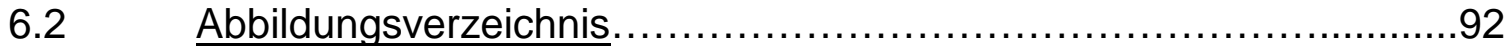

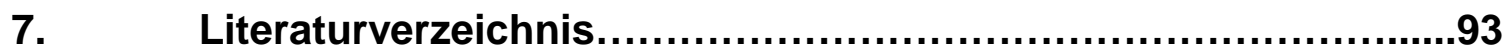




\section{Einleitung}

\subsection{Indikationen für eine Nierenbiopsie}

Seit vielen Jahren gilt die Durchführung einer Nierenbiopsie bei vielen renalen Erkrankungen als unerlässlich, da nicht nur die exakte Diagnosestellung erleichtert wird, sondern auch Aussagen über den Aktivitätsgrad einer Erkrankung und das potentielle Ansprechen auf eine spezielle Therapie getroffen werden können.

Die Aufarbeitung eines Biopsates erfolgt durch Lichtmikroskopie, Immunofluoreszenz und Elektronenmikroskopie. Haas beschrieb 1997 bei einer Studie mit 288 nativen Biopsien, dass die Elektronenmikroskopie zur Diagnosestellung in $21 \%$ der Fälle unerlässlich war und in weiteren $21 \%$ wichtige Zusatzinformationen lieferte (Haas 1997).

Die Indikationen für eine Nierenbiopsie sind vielfältig (Madaio 1990):

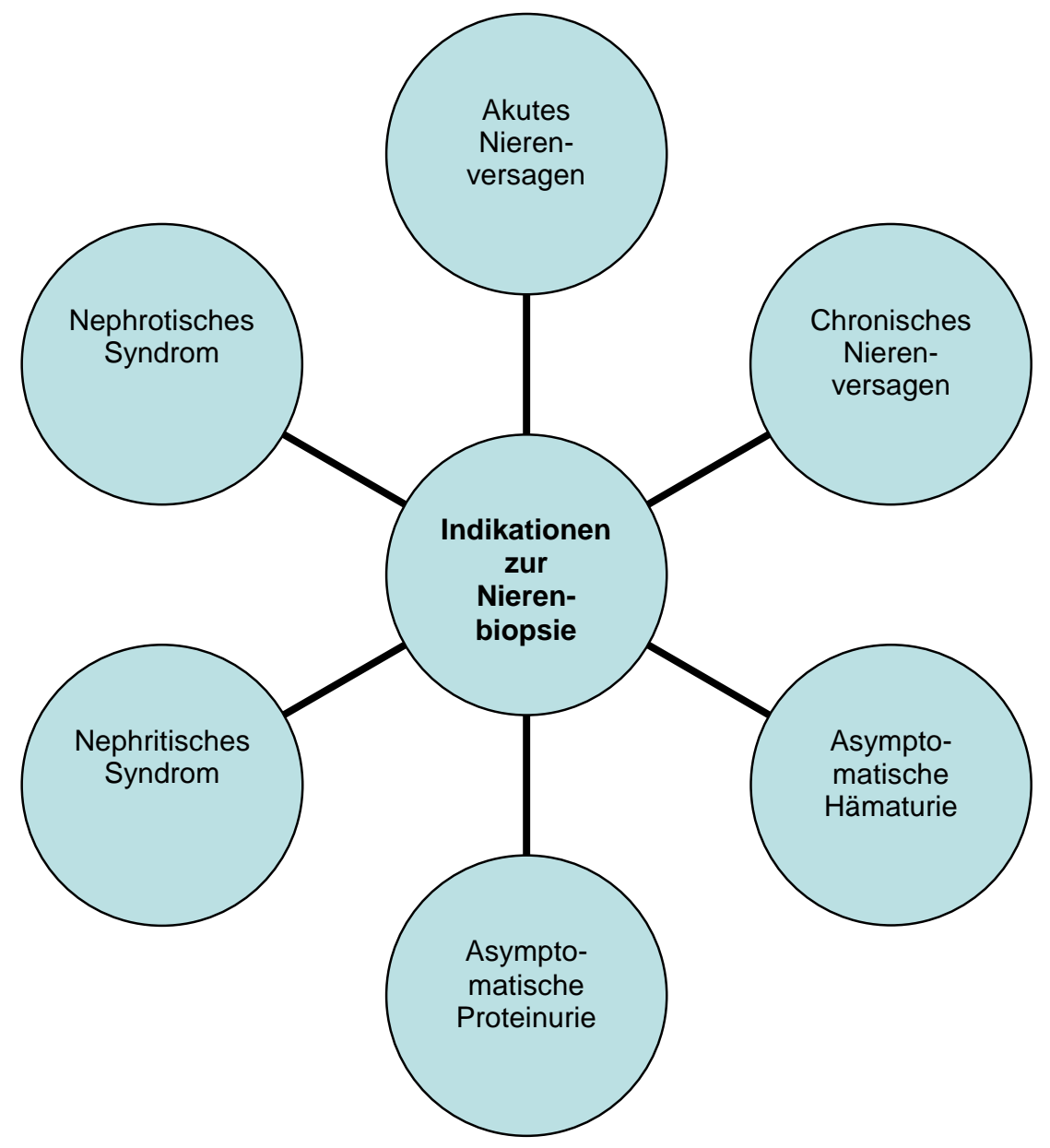

Abb.1:

Übersicht über die absoluten und relativen Indikationen zur Nierenbiopsie 


\subsubsection{Akutes Nierenversagen}

Das akute Nierenversagen beschreibt eine akute, also über wenige Tage oder Wochen auftretende Niereninsuffizienz. Als klinische Auswirkungen kann man vor allem eine Oligo- bis Anurie und ein Ansteigen der Retentionsparameter im Plasma beobachten. Prinzipiell ist das Krankheitsbild durch eine rasche Diagnose und Behandlung aber reversibel und normalerweise bleiben keine bleibenden Schäden zurück.

In den meisten Fällen eines akuten Nierenversagens kann die Diagnose anhand des klinischen Bildes, der Laborwerte und der Ultraschalluntersuchung der Niere getroffen werden. Eine Nierenbiopsie wird hierfür häufig nicht benötigt. In einigen Fällen kann aber trotz dieser Untersuchungen keine sichere Diagnose gestellt werden und die Durchführung einer Nierenbiopsie ist unerlässlich. Zudem konnten Richards et al. in ihrer 1994 veröffentlichten Studie zeigen, dass bei 71 $\%$ der Patienten mit akutem Nierenversagen die weitere Behandlung aufgrund der Ergebnisse aus der Nierenbiopsie geändert wurde (Richards et al. 1994).

\subsubsection{Chronisches Nierenversagen}

Das chronische Nierenversagen, auch chronische Niereninsuffizienz genannt, zeichnet sich durch einen langsamen, über Monate oder Jahre dauernden Verlauf aus. Dabei kommt es zu einer irreversiblen, fortschreitenden Abnahme der Nierenfunktion. Hervorgerufen wird dies durch eine chronische Destruktion von Nephronen, was eine Abnahme des Glomerulumfiltrates bewirkt. Das chronische Nierenversagen kann in eine terminale Niereninsuffizienz münden, mit den letzten Behandlungsmöglichkeiten Hämodialyse oder Nierentransplantation.

Im Gegensatz zum akuten Nierenversagen ist es beim chronischen Nierenversagen oft schwierig, eine Aussage über die Ursache und die Art der chronischen Nierenerkrankung ohne Zuhilfenahme einer Nierenbiopsie zu treffen. Richards et al. zeigten in einer Studie, dass bei chronischem Nierenversagen 45 $\%$ der Patienten aufgrund der Ergebnisse der Nierenbiopsie anders behandelt wurden (Richards et al. 1994). Eine Nierenbiopsie kann also zur Behandlung eines chronischen Nierenversagens durchaus hilfreich sein. 


\subsubsection{Asymptomatische Hämaturie}

Eine asymptomatische Hämaturie ist gekennzeichnet durch eine Hämaturie bei ansonsten unauffälligen Retentionsparametern im Plasma und einer normalen Nierenfunktion. Oft kann ein solches klinisches Bild mit persistierender Hämaturie, einer normalen Kreatininkonzentration im Plasma und einem normalen Blutdruck durch eine glomeruläre Erkrankung, meist aufgrund einer IgA-Nephropathie, einer hereditären Nephritis (Alport-Syndrom) oder eines Syndroms der dünnen Basalmembran (sog. benigne Hämaturie) hervorgerufen sein (Burton 2004).

Die Durchführung einer Nierenbiopsie bei asymptomatischer Hämaturie ist umstritten, da zwar oft die Ursache der Hämaturie gefunden wird, die Therapie sich aber durch diese Erkenntnis selten ändert. Daher wird bei diesem klinischen Bild nur sehr selten biopsiert.

\subsubsection{Asymptomatische Proteinurie ohne Systemerkrankung}

Bei der asymptomatischen Proteinurie besteht eine vermehrte Anreicherung an Proteinen im Urin, ohne eine pathologische Veränderung anderer nierenfunktionsspezifischer Parameter im Plasma oder Urin und ohne eine zugrunde liegende Systemerkrankung.

Eine Nierenbiopsie führt in diesem Fall (Proteinurie unter $1 \mathrm{~g} / 24 \mathrm{~h}$ ) oft nur zu unspezifischen Ergebnissen. Zudem wird die Therapie ähnlich wie bei der asymptomatischen Hämaturie auch bei der asymptomatischen Proteinurie selten durch die Ergebnisse einer Nierenbiopsie beeinflusst. Deshalb ist in beiden Fällen die Indikation einer Nierenbiopsie nur in Ausnahmefällen zu stellen.

\subsubsection{Nephritisches Syndrom}

Das nephritische Syndrom ist definiert über die Kombination von Oligurie, Hämaturie, Erythrozytenzylinder, Azotämie und arteriellen Hypertonus. Im klinischen Bild zeigt sich zudem häufig ein Flankenschmerz. Bei rasch progredientem klinischem Verlauf spricht man auch von einer rapid progredienten Glomerulonephritis.

Bei einem nephritischen Syndrom kann eine Nierenbiopsie in vielen Fällen hilfreich sein: Bei unbekannter Ursache kann so die Diagnose rasch gestellt werden und der Patient so früh wie möglich adäquat therapiert werden. Aber auch der Schweregrad der Erkrankung kann bei bereits bekannter Ursache durch eine 
Nierenbiopsie festgestellt werden, um so bessere Aussagen zur Therapie und Prognose treffen zu können. Als Alternative zur Nierenbiopsie können aber auch spezielle Antikörper im Serum nachgewiesen werden, wie zum Beispiel Antineutrophile zytoplasmatische Antikörper (ANCA) bei der Wegenerschen Granulomatose (Madaio 1990). Trotzdem bleibt die Nierenbiopsie beim nephritischen Syndrom ein wichtiges Verfahren zur Diagnosestellung, vor allem dann, wenn das klinische Bild und die serologischen Laborergebnisse keine eindeutige Diagnose zulassen. Mit einer Nierenbiopsie kann in diesem Fall rasch eine Diagnose gestellt und mit der adäquaten Therapie begonnen werden.

\subsubsection{Nephrotisches Syndrom}

Bei dem nephrotischen Syndrom zeigen sich klinisch eine Proteinurie mit $>3,5 \mathrm{~g} / 24 \mathrm{~h}$ (bezogen auf eine Körperoberfläche von 1,73 $\mathrm{m}^{2}$ ), periphere Ödeme, Hypoproteinämie und Hyperlipoproteinämie durch reaktiv vermehrte Proteinsynthese in der Leber. Die Pathogenese hierbei kann sowohl entzündlicher als auch nicht-entzündlicher Art sein.

Richards et al. zeigten in einer Studie, dass die Erkenntnisse aus der Nierenbiopsie bei $86 \%$ der Patienten mit nephrotischem Syndrom ohne systemische Erkrankung die weitere Behandlung beeinflussten (Richards et al. 1994) und bei diesem klinischen Bild eine Nierenbiopsie durchaus indiziert ist. Bei systemischen Grunderkrankungen wie primärer oder sekundärer Amyloidose oder Diabetes mellitus ist eine Nierenbiopsie dagegen oft nicht indiziert.

\subsection{Durchführung einer Nierenbiopsie}

Bereits im Jahre 1923 wurde über die Durchführung von Nierenbiopsien berichtet (Gwyn 1923). Diese Biopsie wurde in einer offenen, operativen Technik vollzogen. Seitdem wurden mehrere Techniken entwickelt und auch weiterentwickelt, sodass man heute mit minimalem Risiko eine Nierenbiopsie durchführen kann. Im Folgenden sollen die einzelnen Techniken mit ihren Vorund Nachteilen dargestellt werden: 


\subsubsection{Offene Nierenbiopsie}

Das offene, chirurgische Verfahren wurde bereits zu Beginn des 20. Jahrhunderts angewendet und stellt damit das älteste Verfahren dar. Heute wird die offene Nierenbiopsie nur noch selten durchgeführt. Eine der Indikationen zur offenen Nierenbiopsie stellt eine nicht kontrollierbare Gerinnungsstörung dar, alternativ kann in diesem Fall aber auch eine transjuguläre Biopsie durchgeführt werden (Mal et al. 1992). Außerdem besteht auch die Möglichkeit, eine offene Nierenbiopsie durchzuführen, wenn eine einzelne Niere vorliegt oder nach mehreren vergeblichen Versuchen, eine perkutane Biopsie durchzuführen.

Die offene Nierenbiopsie wird im Operationssaal, meist unter Lokalanästhesie durchgeführt. Der Patient befindet sich in leicht nach vorne geneigter Seitenlage für einen optimalen Zugang zum Operationsgebiet (Conger und Sarembock 1961). Nach Durchführung der Lokalanästhesie wird ein Hautschnitt unterhalb der zwölften Rippe gemacht und der operative Zugang zur Niere wird hergestellt. Für die Biopsie selbst wird eine Vim-Silverman-Nadel benutzt (Conger und Sarembock 1961). Der Patient wird aufgefordert die Luft in tiefer Inspiration anzuhalten, sodass die Niere in das Operationsgebiet tritt und gut erreichbar ist. So kann unter direkter Sicht die Biopsie mit der Biopsienadel entnommen werden. Nach Entnahme der Biopsie wird die Wunde wieder geschlossen.

Der Vorteil einer offenen Nierenbiopsie besteht vor allem darin, dass auftretende Blutungen sofort unter direkter Sicht gestillt werden können. Dies ist vor allem für Patienten mit unkontrollierbaren Gerinnungsstörungen von Vorteil. Allerdings kann die Operation selbst auch zu Blutungen führen, sodass eine der Hauptkomplikationen nach wie vor die Blutungsgefahr darstellt. Zudem birgt auch eine Vollnarkose, sofern sie zur Anwendung kommt, Risiken. Des Weiteren stellt die offene Nierenbiopsie einen stark invasiven Eingriff dar und sollte deshalb nur zur Anwendung kommen, wenn die Alternativen genau betrachtet wurden.

\subsubsection{Laparoskopische Nierenbiopsie}

Die laparoskopische Nierenbiopsie gehört zu den jüngeren Verfahren und wurde in mehreren Studien als Alternative zu der offenen Nierenbiopsie beschrieben (Gimenez et al. 1998, Gupta et al. 2000). Gimenez et al. zeigten in einer Studie an 32 Patienten, dass die laparoskopische Nierenbiopsie ein sehr sicheres, minimal-invasives Verfahren ist für Patienten, bei denen eine perkutane Biopsie 
kontraindiziert ist. Biopsiert wurden in dieser Studie Personen, bei denen das perkutane Verfahren mehrfach vergeblich durchgeführt worden war, Patienten mit einer einzelnen Niere, mit einer Gerinnungsstörung, mit multiplen bilateralen renalen Zysten und Patienten, bei denen die physischen Voraussetzungen für eine perkutane Biopsie zum Beispiel aufgrund von Adipositas oder zerebraler Lähmung nicht gegeben waren. Alle 32 Biopsien wurden erfolgreich durchgeführt und ausreichend Gewebe konnte gewonnen werden. Die Verweildauer im Krankenhaus und die Komplikationsrate waren gering (Gimenez et al. 1998).

Für die Biopsie wird der Patient in Seitenlage gebracht und in Vollnarkose versetzt (Gimenez et al. 1998). Der Zugang zu der Niere erfolgt zum einen mit einem laparoskopischen Zugang über dem Darmbeinkamm in der hinteren Axillarlinie, zum anderen über einen zweiten laparoskopischen Zugang auf der selben Höhe in der vorderen Axillarlinie. Im retroperitonealen Fettgewebe wird der untere Pol der Niere stumpf freipräpariert und die Biopsien werden entnommen (Gimenez et al. 1998). Nachdem sichergestellt ist, dass keine Blutung vorliegt, kann die Haut mit einer absorbierbaren Naht verschlossen werden.

Da die laparoskopische Nierenbiopsie noch ein sehr junges Verfahren ist, bleibt abzuwarten, ob zukünftige Studien die Erfolge dieser Behandlung bestätigen können. Diese Methode wird aber schon jetzt von einigen Autoren als Alternative zur offenen Biopsie angesehen (Gimenez et al. 1998, Gupta et al. 2000).

\subsubsection{Transjuguläre Nierenbiopsie}

Genauso wie die laparoskopische ist auch die transjuguläre Nierenbiopsie ein jüngeres Verfahren. Die transjuguläre Nierenbiopsie ist eine Abwandlung der transjugulären Leberbiopsie (Meyrier 2005). Durchgeführt wird die transjuguläre Nierenbiopsie nur, wenn die perkutane Nierenbiopsie kontraindiziert ist oder eine Multiorganbiopsie, zum Beispiel gemeinsam mit einer Leber- oder Herzbiopsie, durchgeführt werden soll. Eine von Cluzel et al. durchgeführte Studie hat gezeigt, dass die transjuguläre Nierenbiopsie eine etwa gleich große Erfolgsquote in Hinsicht auf die Gewinnung von renalem Gewebe hat wie das perkutane Verfahren und nur eine unwesentlich höhere Komplikationsrate (Cluzel et al. 2000). In dieser Studie wurden im Zeitraum von 1993 bis 1998 transjuguläre Nierenbiopsien an 400 Patienten mit einer Kontraindikation für die perkutane Nierenbiopsie durchgeführt (Gerinnungsstörungen, unkontrollierter Hypertonus, 
Adipositas, einzelne Niere, zusätzliche Biopsie von Leber oder Herz) und verglichen mit 400 Patienten, bei denen eine perkutane Nierenbiopsie durchgeführt wurde. In dieser Studie ergaben sich vier Komplikationen mit klinischen Folgen bei der transjugulären und drei bei der perkutanen Nierenbiopsie, wobei das perkutane Verfahren meist ohne Ultraschallüberwachung durchgeführt wurde und die Komplikationsrate mit Zuhilfenahme von Ultraschall hätte verbessert werden können (Cluzel et al. 2000).

Auch Rychlík et al. konnten in einer 2001 veröffentlichten Studie an 67 Patienten zeigen, dass die transjuguläre Nierenbiopsie bei richtiger Durchführung ein wichtiges diagnostisches Verfahren für Patienten mit Kontraindikationen zur perkutanen Nierenbiopsie ist. Zudem konnten auch hier kombinierte Leber- und Nierenbiopsien durchgeführt werden (Rychlík et al. 2001).

In einer weiteren Studie von Fine et al. konnte gezeigt werden, dass die transjuguläre Nierenbiopsie bei adipösen Patienten ein relativ sicheres, zuverlässiges und minimal-invasives Verfahren mit gutem diagnostischem Erfolg ist (Fine et al. 2004). Bei diesen Patienten konnte aufgrund ihrer körperlichen Voraussetzung keine perkutane Nierenbiopsie durchgeführt werden. In dieser Studie zeigte sich, dass bei $89,2 \%$ der 37 Patienten eine histopathologische Diagnose anhand des gewonnenen Gewebes gemacht werden konnte und es bei nur einem Patienten eine Komplikation mit klinischen Folgen gab. Die transjuguläre Nierenbiopsie wurde aufgrund dieser Ergebnisse bei adipösen Patienten als Alternative zu einer laparoskopischen Nierenbiopsie unter Vollnarkose angesehen.

Auch bei Hochrisiko-Patienten (Abbott et al. 2002, Thompson et al. 2004) und bei Patienten mit Leberzirrhose und renalen Funktionsstörungen (Jouet et al. 1996) konnte gezeigt werden, dass die transjuguläre Nierenbiopsie ein sehr sicheres und nützliches Verfahren ist, mit dem wichtige Informationen für die weitere Behandlung gewonnen werden können.

Meist wird die transjuguläre Nierenbiopsie in der radiologischen Abteilung durchgeführt. Zur Durchführung dieser Biopsie befindet sich der Patient in Rückenlage (Mal et al. 1992). Nach der Lokalanästhesie wird die V. jugularis interna punktiert und ein Gefäßdilatator eingeführt. Anschließend wird ein Führungsdraht unter Röntgenkontrolle in die V. cava inferior eingebracht. Über 
diesen Führungsdraht wird der Biopsiekatheter geschoben, in der V. renalis dexter platziert und in den unteren Nierenpol gedrückt. Mit $2 \mathrm{ml}$ Kontrastmittel wird die Position überprüft. Anschließend wird die Biopsienadel mit Kochsalzlösung gefüllt und in den Katheter eingeführt. Die Nadel wird mit einer mit Kochsalzlösung gefüllten Spritze verbunden und unter Vakuum-Aspiration unter der Spitze des Katheters hervorgeschoben und wieder zurückgezogen. So kann die Gewebsprobe in der Nadel, dem Katheter oder der mit Kochsalzlösung gefüllten Spritze erhalten werden. In neueren Studien wurde bei Schwierigkeiten die rechte Niere zu biopsieren alternativ auch die Biopsie der linken Nieren durchgeführt (Fine et al. 2004).

Bei der transjugulären Nierenbiopsie können auch mehrere Biopsate entnommen werden. Mit jedem Biopsiezylinder steigt jedoch die Gefahr vor allem für Blutungskomplikationen, da die Biopsienadel der Kapsel immer näher kommt und so das Risiko einer Perforation der Kapsel steigt (Mal et al. 1992).

Trotz der Ergebnisse aus den oben genannten Studien wird die transjuguläre Nierenbiopsie aber nicht das perkutane Verfahren ersetzen können aufgrund höherer Kosten (Meyrier 2005), eines höheren Zeitaufwandes und auch der Strahlenbelastung, der der Patient während der Untersuchung ausgesetzt wird. Allerdings stellt es eine gute Alternative zu dem offenen oder laparoskopischen Verfahren dar, da es minimal-invasiv ist und sehr zuverlässig ausreichende Mengen an Gewebe gewonnen werden können (Cluzel et al. 2000). Dieses Verfahren ist damit vor allem dann indiziert, wenn keine perkutane Biopsie durchgeführt werden kann, also bei Gerinnungsstörungen, Adipositas, einer einzelnen Niere, unkontrollierbarem Hypertonus oder mehrfachem Versagen des perkutanen Verfahrens. Außerdem kann mit der transjugulären Nierenbiopsie auch eine Leber- oder Herzbiopsie kombiniert werden, weshalb bei Multiorganbiopsien dieses Verfahren zur Anwendung kommt. Eine Kontraindikation zur transjugulären Nierenbiopsie liegt vor allem bei anatomischen Besonderheiten der Nierengefäße vor.

\subsubsection{Perkutane Nierenbiopsie}

Die perkutane Nierenbiopsie gilt heute als Standardmethode, da sie, von erfahrener Hand durchgeführt, für den Patienten einen minimal-invasiven Eingriff darstellt und die Risiken schwerer Nebenwirkungen eher gering sind. Zudem ist 
der finanzielle Aufwand dieses Verfahrens weitaus geringer als bei den oben genannten Techniken.

Seit den fünfziger Jahren des zwanzigsten Jahrhunderts wird das Verfahren der perkutanen Nierenbiopsie zunehmend angewendet. Mit der Einführung von begleitenden bildgebenden Verfahren, wie zum Beispiel Ultraschall, konnte das perkutane Verfahren immer sicherer und gezielter eingesetzt werden. Zusätzlich wurden automatisierte Geräte entwickelt, die die Durchführung der Biopsie erleichterten. Nyman et al. veröffentlichten 1997 eine Studie, in der gezeigt wurde, dass ein Arzt, der erfahren ist im Umgang mit Ultraschallgeräten, die diagnostische Präzision gegenüber der manuellen Technik verbessern und das Komplikationsrisiko senken kann (Nyman et al. 1997).

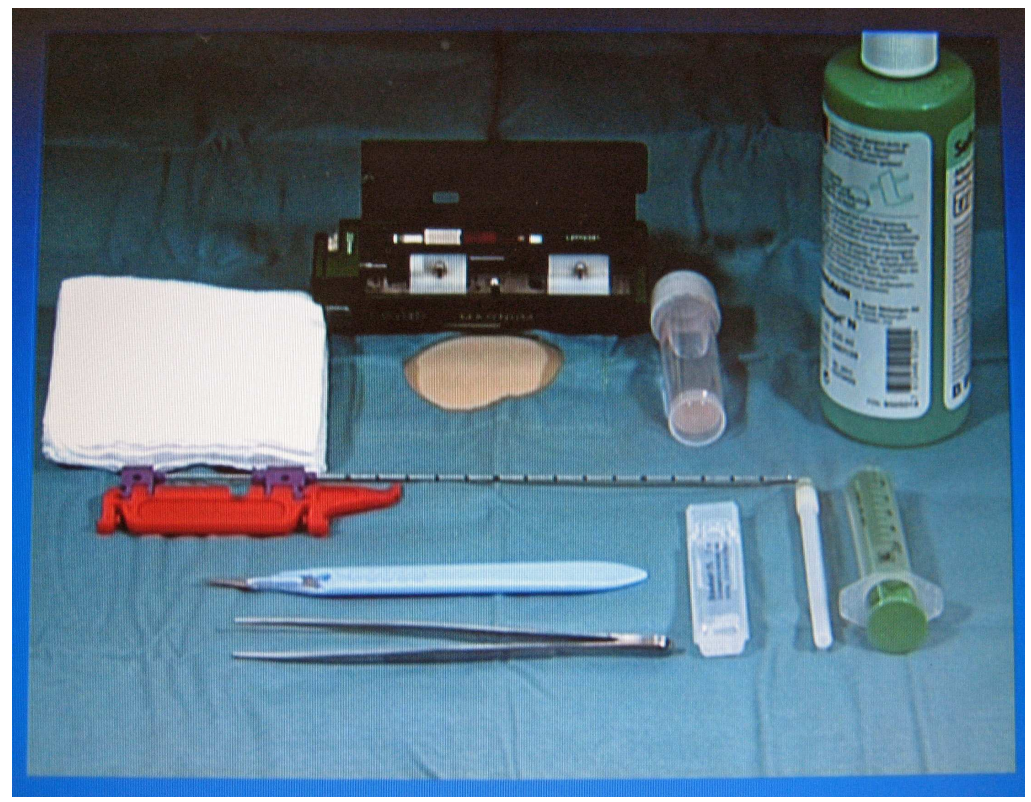

Abb.2:

Materialien zur Durchführung einer Nierenbiopsie: Desinfektionsmittel, Materialien für Lokalanästhesie, Biopsiegerät und Biopsienadel, Biopsiegefäß, Skalpell

Zur Durchführung einer perkutanen Nierenbiopsie (Wiseman et al. 1990) sollte der Patient auf dem Bauch liegen. Die Haut wird desinfiziert und steril abgedeckt. Mit dem Ultraschall wird der untere Pol der Niere dargestellt und die Stelle an der Haut markiert, an der die Biopsienadel die Haut durchdringen soll. Anschließend wird unter Ultraschallüberwachung in die Haut, die Subkutis und das perirenale Gewebe ein Lokalanästhetikum injiziert. Nun erfolgt ein kleiner Hautschnitt, um den Zugang für die Biopsienadel zu erleichtern. Dann wird die Biopsienadel unter Ultraschallkontrolle zum unteren Pol der Niere geführt. Das Vorschieben der Nadel erfolgt bis zum Erreichen der Nierenkapsel. Anschließend dringt die automatisierte Biopsievorrichtung knapp durch die Kapsel und Nierengewebe wird 
entnommen. Dazu dringt die Nadel in das Nierengewebe vor. Über diese Nadel schiebt sich die sie umgebende Kanüle und umgibt so das in der Nadel befindliche Biopsat. Das Nierengewebe kann so entnommen werden und es können auf diesem Wege auch mehrere Gewebeproben aus der Niere entnommen werden. Nach dem Eingriff wird der Patient stationär überwacht und die Niere nochmals mit Ultraschall auf eventuelle Blutungen untersucht.

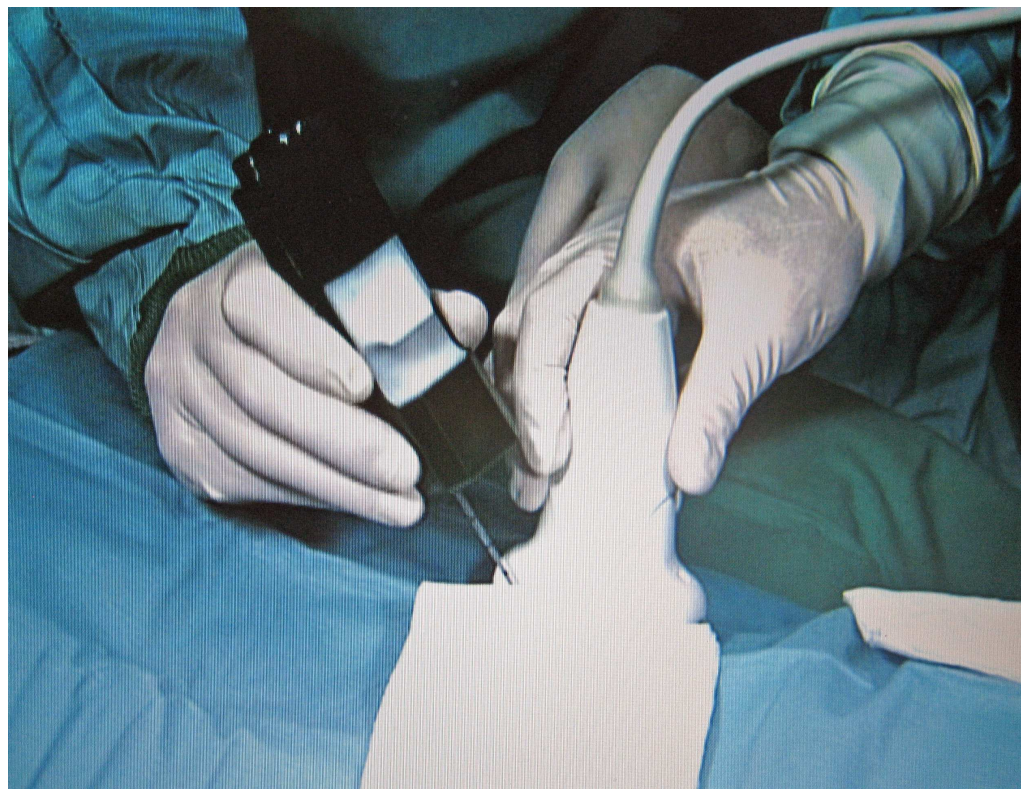

Abb. 3:

Durchführung einer perkutanen Nierenbiopsie unter Ultraschallüberwachung

Obwohl die perkutane Nierenbiopsie heute das Standardverfahren darstellt, gibt es einige Kontraindikationen zu deren Durchführung (Burton 2004). Dazu gehören eine unkontrollierbare Gerinnungsstörung, sehr kleine Nieren mit einem fortgeschrittenen chronischen Schaden, ein Hypertonus, der nicht medikamentös eingestellt werden kann, multiple bilaterale Zysten, ein renaler Tumor, Hydronephrose, eine aktive renale oder perirenale Infektion oder ein unkooperativer Patient. Auch Adipositas ist bei ausgeprägten Fällen eine Kontraindikation für die Durchführung einer perkutanen Nierenbiopsie. Früher galt auch eine singuläre Niere als Kontraindikation zur perkutanen Nierenbiopsie. Studien haben allerdings gezeigt, dass Patienten ohne sonstige Risikofaktoren mit einer singulären Niere durchaus biopsiert werden können (Mendelssohn und Cole 1995).

Bei vorhandenen Kontraindikationen ist das Risiko für eine schwerwiegende Komplikation erhöht und man sollte auf eine andere Technik zurückgreifen, mit 
der die Gefahr einer Komplikation im jeweiligen Fall besser kontrolliert werden kann.

Trotzdem haben viele Studien gezeigt, dass unter Ausschluss der Kontraindikationen eine perkutane Nierenbiopsie ein sehr sicheres Verfahren ist, bei dem in den meisten Fällen ausreichend Gewebe gewonnen werden kann, um eine Diagnose zu stellen (Hergesell et al. 1998).

Die perkutane Nierenbiopsie hat sich in vielen Studien mit vielen Patienten, sowohl bei nativen Nieren als auch bei Transplantatnieren als diagnostisch sinnvolles Verfahren erwiesen, das von den Patienten im Normalfall gut toleriert wird und aufgrund der Ultraschallkontrolle und automatisierter Biopsiegeräte auch mit einem eher geringen Risiko verbunden ist, wie zum Beispiel auch Meola et al. in einer Studie zeigen konnten (Meola et al. 1994). Von erfahrener Hand durchgeführt an Patienten, die keine Kontraindikationen für einen solchen Eingriff haben, ist die perkutane Nierenbiopsie also ein relativ günstiges Verfahren mit einem kurzen stationären Aufenthalt und relativ großer Sicherheit und gilt deshalb auch bis heute, trotz neuerer Verfahren, als Standardmethode für die Nierenbiopsie. Auch bei Kindern konnte gezeigt werden, dass die ultraschallgesteuerte perkutane Nierenbiopsie eine zuverlässige Methode mit geringer Komplikationsrate und großem diagnostischem Wert ist (Feneberga et al. 1998).

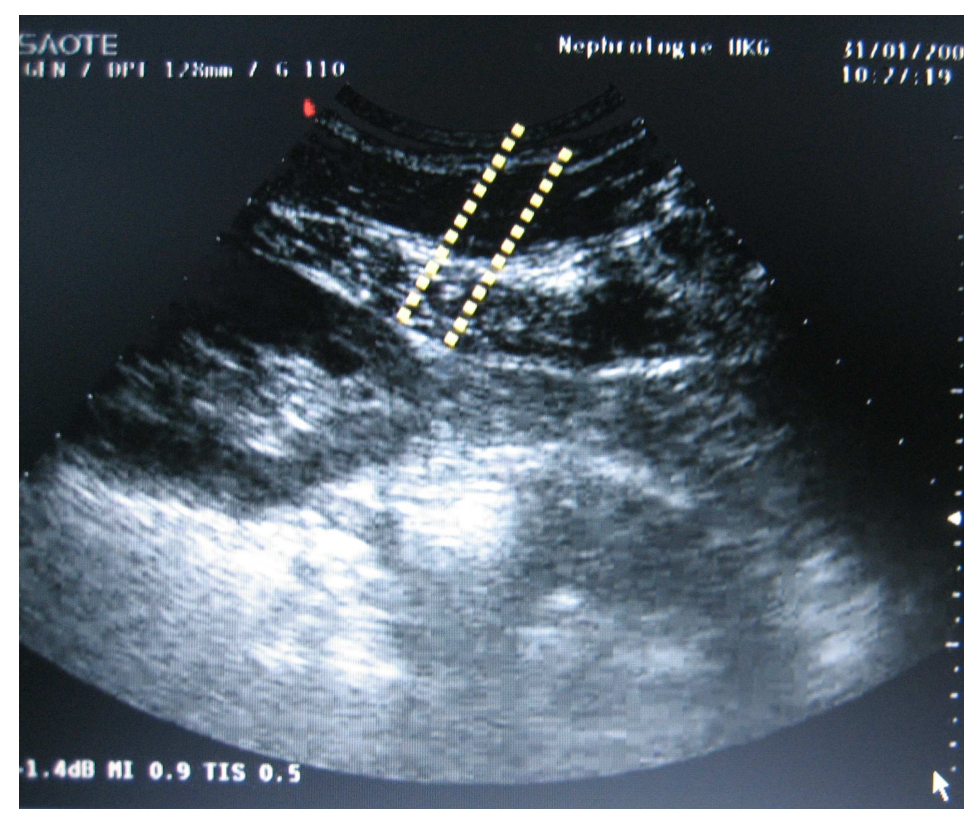

Abb. 4:

Ultraschallgesteuerte Punktion der Niere. Die gelben Striche zeigen den Stichkanal 


\subsection{Komplikationen einer Nierenbiopsie}

Durch die Weiterentwicklungen in der Biopsietechnik sind Nierenbiopsien zwar sicherer geworden, ein gewisser Prozentsatz an Patienten weist aber dennoch eine Komplikation auf, die auf die Biopsie zurückzuführen ist. $\mathrm{Zu}$ den Komplikationen, die bei einer Nierenbiopsie auftreten können, zählen Makrohämaturie, perirenale oder auch intrakapsuläre Hämatome, arterio-venöse Fisteln und ein Hämoglobin ( $\mathrm{Hb}$ )-Abfall. Einen $\mathrm{Hb}$-Abfall von $1 \mathrm{~g} / \mathrm{dl}$ zeigen allerdings etwa $50 \%$ der Patienten, wovon die meisten einen ansonsten komplikationslosen Verlauf aufweisen (Burton 2004, Whittier und Korbet 2004 b). Jörstad et al. erhoben in einer Studie mit 173 perkutanen Nierenbiopsien an 158 Patienten die auftretenden Komplikationen. 57 Patienten wiesen eine Komplikation auf. 23\% der Patienten hatten ein Hämatom, 8\% eine arteriovenöse Fistel und 6\% eine renale Infarzierung (Jörstad et al. 1984).

Viele Komplikationen verlaufen klinisch eher unauffällig und erfordern keine Intervention. Eine Makrohämaturie zum Beispiel bessert sich meist innerhalb weniger Stunden nach einer Biopsie spontan. Allerdings kann bei Persistieren einer solchen Makrohämaturie oder bei dem Auftreten anderer schwerer Blutungskomplikationen die Verabreichung von Bluttransfusionen erforderlich werden.

Bis heute ist es relativ schwierig, das Auftreten einer Blutungskomplikation bei Nierenbiopsien adäquat vorauszusagen. Es sind zwar Risikofaktoren, wie zum Beispiel Gerinnugsstörungen, arterieller Hypertonus oder renale Insuffizienz bekannt, nach wie vor kann aber nicht erfasst werden, welche Patienten, vor allem auch aus diesen Gruppen, besonders gefährdet sind, eine Blutungskomplikation zu erleiden.

Davis und Chandler untersuchten in einer Studie an 120 Patienten mit einer Transplantatniere den Zusammenhang von Gerinnungsparametern und dem Auftreten von Blutungskomplikationen nach Nierenbiopsien (Davis und Chandler 1995). Die meisten Gerinnungsparameter, wie Blutungszeit, Prothrombinzeit, Partielle Thromboplastinzeit (PTT) und Thrombozytenzahl hatten in dieser Studie keinen nachweisbaren Einfluss auf Blutungskomplikationen. Zusätzlich wurde noch der Thromboelastograph (TEG) bestimmt. Und es konnte gezeigt werden, dass ein abnormaler TEG mit einem erhöhten Blutungsrisiko assoziiert war. 
Trotzdem ist es auch heute noch nicht möglich, das individuelle Blutungsrisiko verlässlich vorauszusagen. In dem Bestreben, eine sichere Nachweismethode hierfür zu bekommen, wurde lange Zeit die Blutungszeit bestimmt. Dieses Verfahren wurde aber mittlerweile vielerorts wieder verlassen, da sich keine prognostische Bedeutung zeigen ließ (Lind 1991). Es gab aber auch Studien, die belegten, dass die Bestimmung der Blutungszeit zusammen mit der Thrombozytenzahl, dem Hämatokrit und der Familienanamnese für Blutungsrisiken ein relativ gutes Verfahren darstellt, um ein erhöhtes Blutungsrisiko im Zusammenhang mit Nierenbiopsien zu detektieren (Mattix und Singh 1999).

Da es sehr wichtig ist, mögliche Komplikationen frühzeitig zu erkennen und zu behandeln, untersuchten Whittier und Korbet (2004 b) in einer Studie den Zeitpunkt des Auftretens von Komplikationen nach einer Nierenbiopsie und die Art der Komplikationen. In dieser Studie wurde im Zeitraum von 1983 bis 2002 an 750 Patienten eine perkutane Nierenbiopsie durchgeführt. Bei 13\% der Patienten trat eine Komplikation auf.

Das Auftreten einer Komplikation in dieser Studie war unabhängig von Alter, Geschlecht und Blutungszeit. Auffällig war jedoch, dass Patienten mit einer Komplikation vor der Nierenbiopsie eine höhere Konzentration an Kreatinin im Serum hatten als Patienten, bei denen die Biopsie komplikationslos verlief. Der Serumkreatininspiegel war somit in dieser Studie die einzige Variable, die eine höhere Wahrscheinlichkeit für das Auftreten von Komplikationen anzeigen konnte. Bei $6,7 \%$ der Patienten traten kleinere, klinisch unauffällige Komplikationen auf, bei $6,4 \%$ traten klinisch auffällige Komplikationen auf, die behandelt werden mussten. Makrohämaturie war mit $57 \%$ die häufigste Komplikation. In den meisten Fällen wurde eine Komplikation, die eine Intervention erforderte, mit Bluttransfusionen behandelt. Die meisten Komplikationen und alle behandlungsbedürftigen Komplikationen traten innerhalb von 24 Stunden auf, was auch als idealer Überwachungszeitraum angesehen wurde (Whittier und Korbet 2004 b).

Whittier und Korbet fanden zudem heraus, dass 33\% der Komplikationen nach acht Stunden auftreten und mit einem kurzen Beobachtungszeitraum übersehen würden, weshalb der Überwachungszeitraum auf mindestens 24 Stunden ausgedehnt werden sollte (Whittier und Korbet 2004 a). 
Trotz der ständigen Weiterentwicklung der Technik bei Nierenbiopsien mit besseren und sichereren Verfahren, treten also nach wie vor immer wieder Komplikationen auf und es gibt noch keine zufriedenstellende Methode, um das individuelle Blutungsrisiko vorauszusagen. Bei vielen Faktoren, wie Blutungszeit, PTT oder Plasmakreatininspiegel, konnte ein tatsächlicher Zusammenhang nach wie vor nicht ausreichend bewiesen werden. Ein weiterer möglicher Faktor, um das individuelle Risiko vorhersagen zu können, könnte der platelet function analyzer (PFA)-100 ${ }^{\mathrm{TM}}$ sein, der das Blutungsrisiko verlässlicher vorhersagen soll als dies zum Beispiel bei der Blutungszeit der Fall ist.

\subsection{Platelet function analyzer ( PFA ) $-100^{\mathrm{TM}}$}

Der platelet function analyzer (PFA)-100 ${ }^{\mathrm{TM}}$ stellt eine relativ neue Methode zur invitro-Messung der Thrombozytenfunktion dar. Hierfür wird venöses Vollblut benötigt, das mit Citrat antikoaguliert wird. Das Blut fließt per Unterdruck durch eine Kapillare, die ein kapilläres Blutgefäß nachahmen soll, zu einer kollagenbesetzten Membran, die in ihrer Mitte eine $147 \mu \mathrm{m}$ große Öffnung besitzt. Zusätzlich befinden sich auf der Membran entweder $10 \mu \mathrm{g}$ Epinephrin (EPI) oder $50 \mu \mathrm{g}$ Adenosin-5'-Diphosphat (ADP). Während des Blutflusses setzen sich die Thrombozyten an dem Kollagen ab und werden durch das EPI oder ADP aktiviert. So wird die Öffnung in der Membran langsam verschlossen und der Blutfluss kommt zum Erliegen, wobei die Zeit bis zum Stillstand des Blutflusses von einem Mikroprozessor gemessen wird. Die Referenzintervalle liegen bei 85 bis $165 \mathrm{sec}$ für Kollagen/Epinephrin und 70-120 sec für Kollagen/ADP. Mit diesem Verfahren kann mit relativ geringem Zeitaufwand, auch ohne speziell geschultes Personal, die Thrombozytenfunktion bestimmt werden. Hierfür ist lediglich eine Blutentnahme nötig. Somit ist dieses Verfahren weniger invasiv und für den Patienten angenehmer, als zum Beispiel der Test nach Ivy, bei dem zur Bestimmung der Blutungszeit zunächst eine Hautinzision gemacht werden muss. Der PFA-100 ${ }^{\mathrm{TM}}$ ist seit seiner Einführung sehr häufig Gegenstand von Studien gewesen. So haben zum Beispiel Fressinaud et al. 1998 in einer Studie gezeigt, dass PFA-100 ${ }^{\mathrm{TM}}$ bei Patienten mit von-Willebrand-Krankheit (VWD) eine sehr hohe Sensitivität besitzt und hier sogar besser geeignet ist als die Bestimmung 
der Blutungszeit (Fressinaud et al. 1998). In dieser Studie zeigte sich, dass bei normalem PFA-100 ${ }^{\mathrm{TM}}$ die Diagnose einer VWD ausgeschlossen werden kann, während bei verlängerter Verschlusszeit weitere Untersuchungen, wie zum Beispiel die Durchführung des vWF (von-Willebrand-Faktor)-Antigens, durchgeführt werden mussten.

Favaloro et al. zeigten in ihrer Studie ebenfalls eine hohe Sensitivität des PFA$100^{\mathrm{TM}}$, weshalb Favaloro ebenfalls zu dem Schluss kam, dass die Bestimmung des PFA-100 ${ }^{\mathrm{TM}}$ als Screening Methode für vWD geeignet ist. Zudem wurden in dieser Studie weitere Einflüsse untersucht. So konnte gezeigt werden, dass die Therapie mit Deamino-8-D-Arginin-Vasopressin (DDAVP) bei Patienten mit vWF durch PFA-100 ${ }^{\mathrm{TM}}$ gut überwacht werden konnte, vor allem in Verbindung mit dem VWF:collagen binding activity assay ( VWF:CBA ), der eine hohe Korrelation mit den Verschlusszeiten des PFA-100 ${ }^{\mathrm{TM}}$ zeigte (Favaloro et al. 2001).

Wuillemin et al. untersuchten in einer Studie den Einfluss des PFA-100 ${ }^{\mathrm{TM}}$ bei Patienten mit Blutungsneigung. Hier zeigte sich, dass PFA-100 ${ }^{\mathrm{TM}}$ eine einfache und gute Testmethode der primären Hämostase darstellt. Allerdings zeigte sich hier auch, dass es Grenzen bei der Bestimmung des PFA-100 ${ }^{\mathrm{TM}}$ gibt: die Konzentration des Citratblutes muss definiert sein, das Blut muss innerhalb von einer halben bis vier Stunden im Labor untersucht werden und Hämatokrit und Thrombozytenzahl dürfen nicht zu gering sein, da ansonsten eine verlängerte Verschlusszeit das Ergebnis verfälscht (Wuillemin et al. 2002). Zu einem ähnlichen Ergebnis kamen Harrison et al., die beschrieben, dass die Bestimmung des PFA-100 ${ }^{\mathrm{TM}}$ innerhalb oben beschriebener Grenzen bei Erkrankungen wie vWD, Bernard-Soulier-Syndrom oder Thrombasthenie Glanzmann eine wichtige Untersuchungsmethode sein kann, da nahezu alle diese Patienten verlängerte Verschlusszeiten aufwiesen (Harrison et al. 1999).

Favaloro kam zu dem Schluss, dass PFA-100 ${ }^{\mathrm{TM}}$ zwar nicht spezifisch ist und auch nicht prognostisch für eine Blutungsstörung genutzt werden kann, dass dessen Bestimmung zusätzlich zu einem normalen Gerinnungslabor bei Verdacht auf vWD aber sehr wohl sinnvoll ist (Favaloro 2001).

Auch der Einfluss von Medikamenten auf PFA-100 ${ }^{\mathrm{TM}}$ wurde untersucht, dabei vor allem der Einfluss von Cyclooxygenasehemmern, wie Aspirin. In mehreren Studien zeigte sich, dass Aspirin lediglich Auswirkungen auf Kollagen/EPI und nicht auf Kollagen/ADP hatte (Kottke-Marchant et al. 1999, Homoncik et al. 2000, 
Marshall et al. 1997, Ortel et al. 2000), wohingegen eine verlängerte Verschlusszeit mit Kollagen/ADP meist auf einen schwerwiegenderen Thrombozytenfunktionsdefekt hinwies (Ortel et al. 2000). Zudem konnte gezeigt werden, dass Heparin keinen Einfluss hat auf die Messergebnisse mit PFA-100 ${ }^{\mathrm{TM}}$ und dass PFA-100 ${ }^{\mathrm{TM}}$ auch fähig ist, eine Thrombozytenaggregationshemmung mit Abciximab plus Aspirin zu detektieren (Kottke-Marchant et al. 1999).

Des Weiteren erhielten Homoncik et al. Hinweise darauf, dass PFA- $100^{\mathrm{TM}}$ auch dafür geeignet sein könnte, die Compliance eines Patienten unter Aspirintherapie zu überwachen (Homoncik et al. 2000).

Madan et al. zeigten auch, dass der Effekt von Glykoprotein-Ilb/llla-Antagonisten mithilfe von PFA-100 ${ }^{\mathrm{TM}}$ schnell und einfach nachgewiesen werden kann (Madan et al. 2001). Ebenso kamen Hézard et al. zu dem Schluss, dass PFA-100 ${ }^{\mathrm{TM}}$ geeignet sein kann zur Überwachung bei Langzeitbehandlung mit GlykoproteinIlb/llla-Antagonisten oder auch zur Entscheidung einer zusätzlichen antiaggreganten Therapie vor perkutaner transluminaler koronarer Angioplastie (PTCA) (Hézard et al. 2000).

Mimidis et al. untersuchten die Aussagekraft von PFA-100 ${ }^{\mathrm{TM}}$ bei Patienten mit akuter Pankreatitis. In dieser Studie konnte gezeigt werden, dass eine Verkürzung der Verschlusszeit häufig bei Patienten mit erhöhtem Thromboserisiko im Rahmen einer akuten Pankreatitis auftritt (Mimidis et al. 2004).

Zudem wurde in einer Studie gezeigt, dass PFA-100 ${ }^{\mathrm{TM}}$ auch sinnvoll sein kann bei der Überwachung der Qualität von Thrombozytenkonzentraten (Borzini et al. 1999).

Da die Wirksamkeit von PFA-100 ${ }^{\mathrm{TM}}$ in vielen Gebieten und auch bei Störungen der Thrombozytenfunktion, bei der von-Willebrand-Krankheit und anderen Hämostasestörungen gezeigt werden konnte, stellt sich die Frage, ob der PFA$100^{\mathrm{TM}}$-Wert auch zur Detektion von Blutungskomplikationen geeignet sein kann. Wir untersuchten die Rolle des PFA-100 ${ }^{\mathrm{TM}}$ bei Blutungskomplikationen nach Nierenbiopsien. 


\section{Patienten und Methoden}

\subsection{Studiendesign und Patienten}

Bei der vorliegenden Untersuchung handelt es sich um eine retrospektive Studie. Wir untersuchten die klinischen Daten retrospektiv anhand von Patientenakten von 100 Patientinnen und Patienten, bei denen im Zeitraum von Juli 2003 bis Oktober 2007 an der Universitätsmedizin Göttingen eine sonographiegesteuerte perkutane Nierenbiopsie durchgeführt wurde.

Erhoben wurden das Geschlecht und Alter der Patienten, der PFA-100 ${ }^{\mathrm{TM}}$-Wert, die Nadeldicke der Biopsienadel, der Hb-Wert vor der Biopsie und im Verlauf bis 24 Stunden danach, der Quickwert, die PTT, Kreatinin, Kreatininclearance und MDRD, die nephrologische Diagnose anhand des Biopsieberichtes, sonstige Erkrankungen (Arterielle Hypertonie, Diabetes mellitus und Zustand nach Nierentransplantation) sowie die stationäre Aufenthaltsdauer. Zudem wurden das Vorhandensein eines Hämatoms, ein Hb-Abfall von mehr als $1 \mathrm{~g} / \mathrm{dl}$ innerhalb der ersten 24 Stunden nach Biopsie, sowie weitere Komplikationen protokolliert. Als weitere Komplikationen wurden gewertet eine nach Biopsie auftretende Makrohämaturie, eine Blutung und ein Hb-Abfall, der die Bereitstellung oder Gabe von Erythrozytenkonzentraten zur Folge hatte.

\subsection{Einschlusskriterien}

In die Studie eingeschlossen wurden alle Patientinnen und Patienten, bei denen in oben genanntem Zeitraum eine sonographisch kontrollierte perkutane Nierenbiopsie durchgeführt und zusätzlich der PFA-100 ${ }^{\mathrm{TM}}$-Wert bestimmt wurde. Zudem musste von jedem Patienten ein Biopsiebericht mit der nephrologischen Diagnose vorliegen. 


\subsection{Methoden}

\subsubsection{PFA-100 ${ }^{\mathrm{TM}}$}

Die Bestimmung erfolgte im Zentrallabor der Universitätsmedizin Göttingen. Der Normwert für PFA Koll/EPI, das in dieser Studie bestimmt wurde, betrug 85- 165 sec.

\subsubsection{Nadeldicke}

Die Nadeldicke der Biopsienadel wurde mit der üblichen Größenangabe in Gauge (G) gemessen. 14G entsprechen der dicksten verwendeten Nadelstärke und wurde bei 45 Patienten benutzt, $16 \mathrm{G}$ bei 35 Patienten, $18 \mathrm{G}$ als dünnste verwendete Nadelstärke kam bei 7 Patienten zum Einsatz. Bei 13 Patienten war die verwendete Nadelstärke nicht dokumentiert und wurde deshalb nicht berücksichtigt.

\subsubsection{Hämoglobin}

Vor der Biopsie wurde der $\mathrm{Hb}$ - Wert bestimmt. Die Bestimmung erfolgte im Zentrallabor der Universitätsmedizin Göttingen. Zudem wurde der Hb-Wert im Verlauf über 24 Stunden nach der Biopsie kontrolliert, um Aussagen über einen eventuellen $\mathrm{Hb}$-Abfall treffen zu können.

Normwert: $12-16 \mathrm{~g} / \mathrm{dl}$ bei Frauen und 13,5 - $17 \mathrm{~g} / \mathrm{dl}$ bei Männern

\subsubsection{Quickwert und PTT}

Beide Werte wurden vor der Biopsie im Rahmen der Gerinnungsdiagnostik bestimmt. Die Bestimmung erfolgte aus Citratblut im Zentrallabor der Universitätsmedizin Göttingen. Die Normwerte waren für den Quick: 70 - 120\%, für die PTT: 26 - $37 \mathrm{sec}$.

\subsubsection{Kreatinin}

Bei allen Patienten wurde vor der Biopsie der Kreatininwert als ein Parameter der Nierenfunktion bestimmt. Die Bestimmung erfolgte im Zentrallabor der Universitätsmedizin Göttingen. Der Normwert betrug: $<1,2 \mathrm{mg} / \mathrm{dl}$ 


\subsubsection{Kreatininclearance und MDRD}

Bei 61 Patienten wurde vor der Biopsie die Kreatininclearance mittels 24 Stunden Sammelurin gemessen. Die Bestimmung erfolgte im Zentrallabor der Universitätsmedizin Göttingen. Als Normwert galt eine Clearance von $80-140$ $\mathrm{ml} / \mathrm{min}$.

Zudem erfolgte die Berechnung der GFR mit der MDRD-Formel. Diese versucht die Kreatininclearance auf der Basis von Alter, Geschlecht, Kreatinin und Hautfarbe abzuschätzen. Allerdings ist die Formel nur zutreffend für Patienten mit durchschnittlicher Konstitution. Der Normwert für die MDRD - Formel war: $>60$ $\mathrm{ml} / \mathrm{min} / 1,73 \mathrm{~m}^{2} \mathrm{KO}$.

\subsubsection{Nephrologische Diagnose}

Die nephrologische Diagnose wurde anhand des Biopsieberichtes erhoben. Insgesamt wurden aus den nephrologischen Diagnosen zehn Gruppen gebildet: Nephrosklerose, FSGS, IgA-Nephritis, Lupusnephritis, membranoproliferative Glomerulonephritis (GN), membranöse GN, Transplantat-Glomerulopathie, minimal change GN, diabetische Nephropathie, andere (kein Anhaltspunkt für GN, Cholesterin-Embolie, Tubulus-Atrophie, akutes Nierenversagen, Amyloidose, Komplementfaktor-C3-positive GN, chronischer interstitieller Schaden, kein Parenchym).

\subsubsection{Hämatom}

Am Tag nach der Biopsie wurde in der Universitätsmedizin Göttingen eine Ultraschalluntersuchung zur Beurteilung von eventuell durch die Biopsie verursachten Hämatomen durchgeführt.

\subsection{Statistische Methoden}

Die Auswertung erfolgte mithilfe der Software STATISTICA (V 8.0.360, StatSoft). Neben der deskriptiven Statistik kamen weitere Testverfahren zur Anwendung:

1. Der t-Test für unabhängige Stichproben zu einem Niveau von $5 \%$. 
Dieses Testverfahren eignet sich zu einem Vergleich von Mittelwerten verschiedener Stichproben. Der Vorteil dieses Verfahrens ist, dass auch kleinere Stichproben gut auf Signifikanz getestet werden können. Voraussetzung für die Gültigkeit des t-Tests ist, dass eine Normalverteilung der Variablen vorliegt.

2. Pearsons Chi-Quadrat-Test zu einem Niveau von $5 \%$.

Dieses Testverfahren dient dazu, Zusammenhänge zwischen kategoriellen Variablen zu berechnen. Der Test beruht auf der Tatsache, dass erwartete Häufigkeiten berechnet werden können. Die Signifikanz nimmt zu, je weiter das Untersuchungsergebnis von der erwarteten Häufigkeit abweicht. Voraussetzung ist, dass die erwarteten Häufigkeiten nicht zu klein sein dürfen. Die Variablen werden in zweidimensionalen Häufigkeitstabellen aufgetragen, bei zwei dichotomen Variablen handelt es sich hierbei um 2x2 Tafeln. 


\section{Ergebnisse}

\subsection{Allgemein}

Untersucht wurden in unserer Studie 100 Patienten und Patientinnen, bei denen im Zeitraum von Juli 2003 bis Oktober 2007 eine Nierenbiopsie in der Universitätsmedizin Göttingen durchgeführt wurde. Die Auswertung erfolgte retrospektiv anhand der Patientenakten.

Unter den Patienten waren 58\% Männer (entsprechend 58 absolut) und 42\% Frauen (42). Das Durchschnittsalter der Patienten lag bei 49,6 Jahren (Median 52 Jahre) mit einem Minimum bei 16 und einem Maximum bei 76 Lebensjahren.

Alle erhobenen Werte wurden auf ihre Beziehung zur Entstehung von Hämatomen nach Biopsie, auf ihre Beziehung zu einem Hb-Abfall $>1 \mathrm{~g} / \mathrm{dl}$ innerhalb von 24 Stunden nach Biopsie und auf ihre Beziehung zu entstandenen Komplikationen untersucht. Dabei wurde zunächst allgemein betrachtet, ob eine symptomatische Komplikation entstanden war und dann nochmals gesondert nach den drei Komplikationsarten Makrohämaturie, Blutung, Hb-Abfall mit klinischen Folgen unterteilt.

Insgesamt zeigten $19 \%$ der Patienten (absolut 19) nach der Biopsie sonographisch einen Hinweis auf ein Hämatom, bei 55\% konnte sonographisch ein Hämatom ausgeschlossen werden (55\%) und bei 26 konnte retrospektiv kein Rückschluss mehr auf ein Hämatom gemacht werden, da der Sonographiebefund unvollständig war $(26 \%)$.

$55 \%$ der Patienten (absolut 55) hatten nach der Biopsie keinen Hb-Abfall $>1 \mathrm{~g} / \mathrm{dll}$. Bei $41 \%(n=41)$ konnte ein $\mathrm{Hb}$-Abfall von mehr als $1 \mathrm{~g} / \mathrm{dl}$ nachgewiesen werden und bei $4 \%(n=4)$ konnten aufgrund fehlender Werte retrospektiv keine Aussagen zu einem eventuellen $\mathrm{Hb}$-Abfall gemacht werden.

Von den 100 untersuchten Patienten hatten 13\% $(n=13)$ eine Komplikation nach Biopsie. 


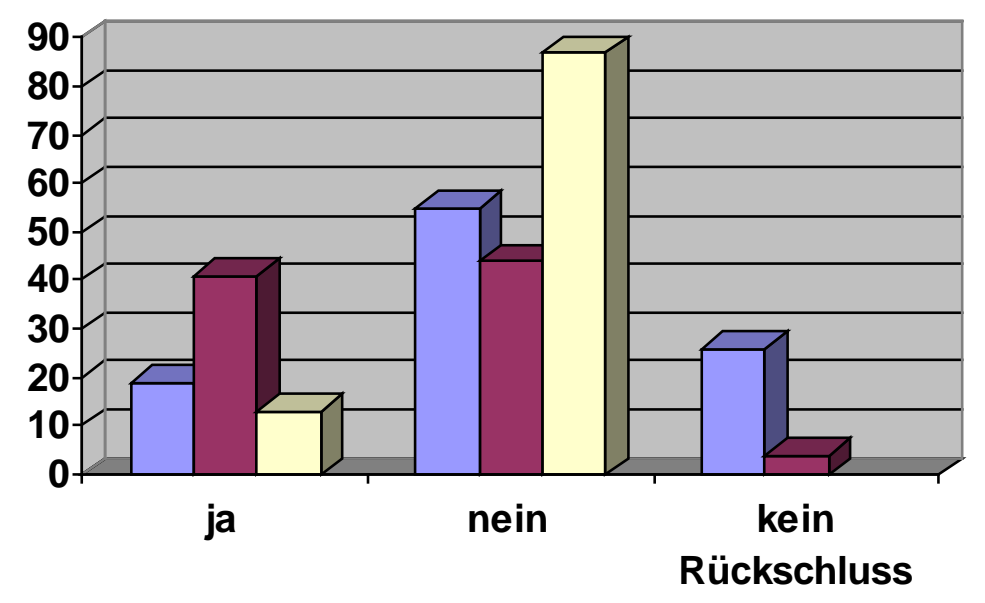

\section{$\square$ Hämatom \\ $\square \mathrm{Hb}-$ Abfall $>1 \mathrm{~g} / \mathrm{dl}$ \\ $\square$ symptomatische Komplikation}

Diese 13 symptomatischen Komplikationen wurden nochmals unterteilt in die drei Komplikationsarten Makrohämaturie bei $5 \%(n=5)$ der Patienten. Diese war allerdings in allen Fällen spontan wieder rückgängig. $3 \%(n=3)$ der Patienten hatten eine Blutung nach Biopsie, darunter befand sich ein Patient, bei dem aufgrund einer großen retroperitonealen Blutung eine operative Hämatomausräumung erfolgen musste. Die übrigen Patienten mit einer symptomatischen Komplikation hatten einen $\mathrm{Hb}$-Abfall, der eine Gabe oder Bereitstellung von Erythrozytenkonzentraten erforderte. Dies entsprach 5\% der Patienten (5 absolut). Es traten keine biopsiebedingten Todesfälle oder Transplantatverluste auf.

Jeder der erhobenen Faktoren wurde geprüft auf seine Beziehung zu den Parametern: Hämatom, Hb-Abfall > $1 \mathrm{~g} / \mathrm{dl}$, Komplikation und Komplikationsart. Da bei gewissen Parametern wie PFA-100 ${ }^{\mathrm{TM}}$, Quickwert und MDRD die Angabe von Werten ober- oder unterhalb einer gewissen Grenze nicht genau möglich ist, wurden in diesen Fällen jeweils zwei Berechnungen gemacht: zum einen mit den möglichst genauen Werten, wobei ober- oder unterhalb der Grenzen gerundet werden musste, zum anderen mit der Unterteilung in Gruppen.

Bei PFA $-100^{\mathrm{TM}}$ können Werte über $300 \mathrm{sec}$ nicht exakt angegeben werden, deshalb wurden einmal zur Berechnung alle Werte über $300 \mathrm{sec}$ mit $300 \mathrm{sec}$ gleichgesetzt. Es erfolgte eine zweite Berechnung in den Gruppen 1: PFA $<85$ 
sec also zu niedrig, 2: PFA 85 - 165 sec also normwertig und 3: PFA > 165 sec also zu hoch.

Ähnlich wurde beim Quickwert verfahren. Werte von über 130\% konnten nicht genau bestimmt werden und wurden mit 130\% gleichgesetzt. Eine zweite Berechnung erfolgte in den Gruppen 1: Quick < 70\% also zu niedrig, 2: Quick 70 - 120\% also normwertig, 3: Quick > 120\% also zu hoch.

Bei der MDRD-Formel können Werte über $60 \mathrm{ml} / \mathrm{min} / 1,73 \mathrm{~m}^{2} \mathrm{KO}$ und unter 20 $\mathrm{ml} / \mathrm{min} / 1,73 \mathrm{~m}^{2} \mathrm{KO}$ nicht exakt angegeben werden. In der ersten Berechnung wurden diese Werte deshalb mit 20 bzw. $60 \mathrm{ml} / \mathrm{min} / 1,73 \mathrm{~m}^{2} \mathrm{KO}$ gleichgesetzt. Eine zweite Berechnung erfolgte in Gruppen, die an der Einteilung der Niereninsuffizienzklassen orientiert waren: Gruppe 1: Niereninsuffizienzgrad I und II, d.h. MDRD > $60 \mathrm{ml} / \mathrm{min} / 1,73 \mathrm{~m}^{2} \mathrm{KO}$, Gruppe 2: Niereninsuffizienzgrad III, also 30 - $60 \mathrm{ml} / \mathrm{min} / 1,73 \mathrm{~m}^{2} \mathrm{KO}$, Gruppe 3: Niereninsuffizienzgrad IV, d.h. $<30$ $\mathrm{ml} / \mathrm{min} / 1,73 \mathrm{~m}^{2} \mathrm{KO}$.

So sollte trotz der Schwierigkeit von exakten Berechnungen ein möglichst genaues Ergebnis erreicht werden.

Alle Signifikanztests wurden zu einem Niveau von 5\% durchgeführt, der p-Wert auf 4 Nachkommastellen gerundet.

Die Nullhypothesen für die t-Tests lauteten:

1) Für Hämatom: Die Patienten mit Hämatomentwicklung nach der Biopsie zeigen keinen Unterschied in der Höhe des Spiegels des jeweiligen Parameters zu den Patienten, die kein Hämatom entwickelten.

2) Für Hb-Abfall> $1 \mathrm{~g} / \mathrm{dl}$ : Die Patienten mit einem Hb-Abfall > $1 \mathrm{~g} / \mathrm{dl}$ in den ersten 24 Stunden nach Biopsie zeigen keinen Unterschied in der Höhe des Spiegels des jeweiligen Parameters zu den Patienten ohne Hb-Abfall > $1 \mathrm{~g} / \mathrm{dl}$.

3) Für Komplikation: Die Patienten mit einer Komplikation nach Biopsie zeigen keinen Unterschied in der Höhe des Spiegels des jeweiligen Parameters zu den Patienten ohne Komplikation.

4) Für Makrohämaturie: Die Patienten mit einer neu aufgetretenen Makrohämaturie zeigen keinen Unterschied in der Höhe des Spiegels des jeweiligen Parameters zu den Patienten ohne Komplikation. 
5) Für Blutung: Die Patienten mit einer Blutung nach Biopsie zeigen keinen Unterschied in der Höhe des Spiegels des jeweiligen zu den Patienten ohne Komplikation.

6) Für klinisch relevanten $\mathrm{Hb}$-Abfall: Die Patienten mit einem klinisch relevanten $\mathrm{Hb}$-Abfall zeigen keinen Unterschied in der Höhe des Spiegels des jeweiligen Parameters zu den Patienten ohne Komplikation.

Die Nullhypothesen für die Chi-Quadrat-Tests nach Pearson lauteten:

1) Für Hämatom: Die Patienten mit Hämatomentwicklung zeigen keinen Unterschied in der prozentualen Verteilung auf die jeweiligen Gruppen des erhobenen Parameters zu den Patienten ohne Hämatom.

2) Für Hb-Abfall $>1 \mathrm{~g} / \mathrm{dl}$ : Die Patienten mit $\mathrm{Hb}$-Abfall $>1 \mathrm{~g} / \mathrm{dl}$ innerhalb der ersten 24 Stunden nach Biopsie zeigen keinen Unterschied in der prozentualen Verteilung auf die jeweiligen Gruppen des erhobenen Parameters zu den Patienten ohne $\mathrm{Hb}$-Abfall $>1 \mathrm{~g} / \mathrm{dl}$.

3) Für Komplikation: Die Patienten mit einer Komplikation nach Biopsie zeigen keinen Unterschied in der prozentualen Verteilung auf die jeweiligen Gruppen des erhobenen Parameters zu den Patienten ohne Komplikation.

4) Für Makrohämaturie: Die Patienten mit einer neu aufgetretenen Makrohämaturie zeigen keinen Unterschied in der prozentualen Verteilung auf die jeweiligen Gruppen des erhobenen Parameters zu den Patienten ohne Komplikation.

5) Für Blutung: Die Patienten mit einer Blutung nach Biopsie zeigen keinen Unterschied in der prozentualen Verteilung auf die jeweiligen Gruppen des erhobenen Parameters zu den Patienten ohne Komplikation.

6) Für klinisch relevanten $\mathrm{Hb}$-Abfall: Die Patienten mit einem klinisch relevanten $\mathrm{Hb}$-Abfall zeigen keinen Unterschied in der prozentualen Verteilung auf die Gruppen des jeweiligen Parameters zu den Patienten ohne Komplikation. 


\section{2 Übersicht über die statistischen Ergebnisse}

Die folgenden Tabellen sollen eine Übersicht über die statistischen Ergebnisse unserer Untersuchung geben. Geprüft wurde jeweils der Zusammenhang zwischen den in der Tabelle angeführten Parametern und Hämatomentstehung, $\mathrm{Hb}$-Abfall, Komplikationsrate und den Komplikationsarten Makrohämaturie, Blutung und klinisch relevantem $\mathrm{Hb}$-Abfall.

Für die nominal skalierten Parameter wurden in den Tabellen die absoluten und die in dieser Gruppe prozentualen Häufigkeiten angegeben.

Für die ordinal skalierten Parameter wurden in der Tabelle die Mittelwerte +/Standardfehler, sowie in Klammer die Stichprobenumfänge angegeben.

Tab. 1: nominal skalierte Parameter und Hämatomentstehung

Tab. 2: nominal skalierte Parameter und Hb-Abfall

Tab. 3: nominal skalierte Parameter und Komplikation

Tab. 4: nominal skalierte Parameter und Makrohämaturie

Tab. 5: nominal skalierte Parameter und Blutung

Tab. 6: nominal skalierte Parameter und klinisch relevanter Hb-Abfall

Tab. 7: ordinal skalierte Parameter und Hämatom

Tab. 8: ordinal skalierte Parameter und Hb-Abfall

Tab. 9: ordinal skalierte Parameter und Komplikation

Tab. 10: ordinal skalierte Parameter und Makrohämaturie

Tab. 11: ordinal skalierte Parameter und Blutung

Tab. 12: ordinal skalierte Parameter und klinisch relevanter Hb-Abfall 


\begin{tabular}{|c|c|c|c|}
\hline Parameter & Kein Hämatom & Hämatom & p-Wert \\
\hline PFA & & & 0,1024 \\
\hline 1 zu niedrig & $5(9,09 \%)$ & $4(21,05 \%)$ & \\
\hline 2 normal & $38(69,09 \%)$ & $8(42,11 \%)$ & \\
\hline 3 zu hoch & $12(21,82 \%)$ & $7(36,84 \%)$ & \\
\hline Geschlecht & & & 0,8001 \\
\hline 1 männlich & $30(54,55 \%)$ & $11(57,89 \%)$ & \\
\hline 2 weiblich & $25(45,45 \%)$ & $8(42,11 \%)$ & \\
\hline Nadeldicke & & & 0,8259 \\
\hline $114 G$ & $27(58,70 \%)$ & $10(58,82 \%)$ & \\
\hline $216 G$ & $18(39,13 \%)$ & $7(41,18 \%)$ & \\
\hline $318 G$ & $1(2,17 \%)$ & $0(0,00 \%)$ & \\
\hline Quick & & & 0,0639 \\
\hline $1<70 \%$ & $1(1,92 \%)$ & $2(10,53 \%)$ & \\
\hline $270-120 \%$ & $36(69,23 \%)$ & $8(42,11 \%)$ & \\
\hline $3>120 \%$ & $15(28,85 \%)$ & $9(47,37 \%)$ & \\
\hline MDRD & & & 0,4387 \\
\hline $1>60 \mathrm{ml} / \mathrm{min} / 1,73 \mathrm{~m}^{2} \mathrm{KO}$ & $15(34,88 \%)$ & $5(35,71 \%)$ & \\
\hline $230-60 \mathrm{ml} / \mathrm{min} / 1,73 \mathrm{~m}^{2} \mathrm{KO}$ & $13(30,23 \%)$ & $2(14,29 \%)$ & \\
\hline $3<30 \mathrm{ml} / \mathrm{min} / 1,73 \mathrm{~m}^{2} \mathrm{KO}$ & $15(34,88 \%)$ & $7(50,00 \%)$ & \\
\hline Arterielle Hypertonie & & & 0,9702 \\
\hline $1 \mathrm{ja}$ & $35(63,64 \%)$ & $12(63,16 \%)$ & \\
\hline 2 nein & $20(36,36 \%)$ & $7(36,84 \%)$ & \\
\hline Diabetes mellitus & & & 0,8955 \\
\hline $1 \mathrm{ja}$ & $8(14,55 \%)$ & $3(15,79 \%)$ & \\
\hline 2 nein & $47(85,45 \%)$ & $16(84,21 \%)$ & \\
\hline Transplantatniere & & & 0,6998 \\
\hline $1 \mathrm{ja}$ & $14(25,45 \%)$ & $4(21,05 \%)$ & \\
\hline 2 nein & $41(74,55 \%)$ & $15(78,95 \%)$ & \\
\hline Nephrologische Diagnose & & & 0,2313 \\
\hline 1 Nephrosklerose & $10(18,18 \%)$ & $4(21,05 \%)$ & \\
\hline 2 FSGS & $11(20,00 \%)$ & $1(5,26 \%)$ & \\
\hline $3 \lg A-N e p h r i t i s$ & $10(18,18 \%)$ & $6(31,58 \%)$ & \\
\hline 4 Lupusnephritis & $2(3,64 \%)$ & $3(15,79 \%)$ & \\
\hline 5 membranoproliferative GN & $2(3,64 \%)$ & $0(0,00 \%)$ & \\
\hline 6 membranöse GN & $0(0,00 \%)$ & $1(5,26 \%)$ & \\
\hline 7 Transplantat - Glomerulopathie & $2(3,64 \%)$ & $1(5,26 \%)$ & \\
\hline 8 minimal change GN & $3(5,45 \%)$ & $0(0,00 \%)$ & \\
\hline 9 diabetische Nephropathie & $0(0,00 \%)$ & $0(0,00 \%)$ & \\
\hline 10 Andere & $15(27,27 \%)$ & $3(15,79 \%)$ & \\
\hline
\end{tabular}

Tab.1 


\begin{tabular}{|c|c|c|c|}
\hline Parameter & Kein Hb-Abfall & Hb-Abfall & p-Wert \\
\hline PFA & & & 0,1773 \\
\hline 1 zu niedrig & $7(12,73 \%)$ & $3(7,32 \%)$ & \\
\hline 2 normal & $38(69,09 \%)$ & $24(58,54 \%)$ & \\
\hline 3 zu hoch & $10(18,18 \%)$ & $14(34,15 \%)$ & \\
\hline Geschlecht & & & 0,5344 \\
\hline 1 männlich & $33(60,00 \%)$ & $22(53,66 \%)$ & \\
\hline 2 weiblich & $22(40,00 \%)$ & $19(46,34 \%)$ & \\
\hline Nadeldicke & & & 0,4222 \\
\hline $114 G$ & $24(50,00 \%)$ & $19(54,29 \%)$ & \\
\hline $216 G$ & $19(39,58 \%)$ & $15(42,86 \%)$ & \\
\hline $318 \mathrm{G}$ & $5(10,42 \%)$ & $1(2,86 \%)$ & \\
\hline Quick & & & 0,5504 \\
\hline $1<70 \%$ & $1(1,89 \%)$ & $2(5,13 \%)$ & \\
\hline $270-120 \%$ & $33(62,26 \%)$ & $26(66,67 \%)$ & \\
\hline $3>120 \%$ & $19(35,85 \%)$ & $11(28,21 \%)$ & \\
\hline MDRD & & & 0,8648 \\
\hline $1>60 \mathrm{ml} / \mathrm{min} / 1,73 \mathrm{~m}^{2} \mathrm{KO}$ & $14(31,11 \%)$ & $10(30,30 \%)$ & \\
\hline $230-60 \mathrm{ml} / \mathrm{min} / 1,73 \mathrm{~m}^{2} \mathrm{KO}$ & $13(28,89 \%)$ & $8(24,24 \%)$ & \\
\hline $3<30 \mathrm{ml} / \mathrm{min} / 1,73 \mathrm{~m}^{2} \mathrm{KO}$ & $18(40,00 \%)$ & $15(45,45 \%)$ & \\
\hline Arterielle Hypertonie & & & 0,7338 \\
\hline $1 \mathrm{ja}$ & $33(60,00 \%)$ & $26(63,41 \%)$ & \\
\hline 2 nein & $22(40,00 \%)$ & $15(36,59 \%)$ & \\
\hline Diabetes mellitus & & & 0,1387 \\
\hline $1 \mathrm{ja}$ & $7(12,73 \%)$ & $10(24,39 \%)$ & \\
\hline 2 nein & $48(87,27 \%)$ & $31(75,61 \%)$ & \\
\hline Transplantatniere & & & 0,1966 \\
\hline $1 \mathrm{ja}$ & $14(25,45 \%)$ & $6(14,63 \%)$ & \\
\hline 2 nein & $41(74,55 \%)$ & $35(85,37 \%)$ & \\
\hline Nephrologische Diagnose & & & 0,4346 \\
\hline 1 Nephrosklerose & $8(14,55 \%)$ & $8(19,51 \%)$ & \\
\hline 2 FSGS & $14(25,45 \%)$ & $5(12,20 \%)$ & \\
\hline $3 \lg \mathrm{A}-\mathrm{Nephritis}$ & $10(18,18 \%)$ & $8(19,51 \%)$ & \\
\hline 4 Lupusnephritis & $2(3,64 \%)$ & $4(9,76 \%)$ & \\
\hline 5 membranoproliferative GN & $0(0,00 \%)$ & $2(4,88 \%)$ & \\
\hline 6 membranöse GN & $3(5,45 \%)$ & $0(0,00 \%)$ & \\
\hline 7 Transplantat - Glomerulopathie & $2(3,64 \%)$ & $2(4,88 \%)$ & \\
\hline 8 minimal change GN & $2(3,64 \%)$ & $2(4,88 \%)$ & \\
\hline 9 diabetische Nephropathie & $1(1,82 \%)$ & $1(2,44 \%)$ & \\
\hline 10 Andere & $13(23,64 \%)$ & $9(21,95 \%)$ & \\
\hline
\end{tabular}

Tab. 2 


\begin{tabular}{|c|c|c|c|}
\hline Parameter & Keine Komplikation & Komplikation & p-Wert \\
\hline PFA & & & 0,8147 \\
\hline 1 zu niedrig & $9(10,34 \%)$ & $1(7,69 \%)$ & \\
\hline 2 normal & $58(66,67 \%)$ & $8(61,54 \%)$ & \\
\hline 3 zu hoch & $20(22,99 \%)$ & $4(30,77 \%)$ & \\
\hline Geschlecht & & & 0,3791 \\
\hline 1 männlich & $49(56,32 \%)$ & $9(69,23 \%)$ & \\
\hline 2 weiblich & $38(43,68 \%)$ & $4(30,77 \%)$ & \\
\hline Nadeldicke & & & 0,3632 \\
\hline $114 G$ & $39(52,70 \%)$ & $6(46,15 \%)$ & \\
\hline $216 G$ & $28(37,84 \%)$ & $7(53,85 \%)$ & \\
\hline $318 G$ & $7(9,46 \%)$ & $0(0,00 \%)$ & \\
\hline Quick & & & 0,6795 \\
\hline $1<70 \%$ & $3(3,57 \%)$ & $0(0,00 \%)$ & \\
\hline $270-120 \%$ & $54(64,29 \%)$ & $7(58,33 \%)$ & \\
\hline $3>120 \%$ & $27(32,14 \%)$ & $5(41,67 \%)$ & \\
\hline MDRD & & & 0,0338 \\
\hline $1>60 \mathrm{ml} / \mathrm{min} / 1,73 \mathrm{~m}^{2} \mathrm{KO}$ & $24(33,80 \%)$ & $1(10,00 \%)$ & \\
\hline $230-60 \mathrm{ml} / \mathrm{min} / 1,73 \mathrm{~m}^{2} \mathrm{KO}$ & $21(29,58 \%)$ & $1(10,00 \%)$ & \\
\hline $3<30 \mathrm{ml} / \mathrm{min} / 1,73 \mathrm{~m}^{2} \mathrm{KO}$ & $26(36,62 \%)$ & $8(80,00 \%)$ & \\
\hline Arterielle Hypertonie & & & 0,5647 \\
\hline $1 \mathrm{ja}$ & $53(60,92 \%)$ & $9(69,23 \%)$ & \\
\hline 2 nein & $34(39,08 \%)$ & $4(30,77 \%)$ & \\
\hline Diabetes mellitus & & 8 & 0,8680 \\
\hline $1 \mathrm{ja}$ & $15(17,24 \%)$ & $2(15,38 \%)$ & \\
\hline 2 nein & $72(82,76 \%)$ & $11(84,62 \%)$ & \\
\hline Transplantatniere & & & 0,9200 \\
\hline $1 \mathrm{ja}$ & $19(21,84 \%)$ & $3(23,08 \%)$ & \\
\hline 2 nein & $68(78,16 \%)$ & $10(76,92 \%)$ & \\
\hline Nephrologische Diagnose & & & 0,5544 \\
\hline 1 Nephrosklerose & $12(13,79 \%)$ & $5(38,46 \%)$ & \\
\hline 2 FSGS & $18(20,69 \%)$ & $1(7,69 \%)$ & \\
\hline $3 \lg A-N e p h r i t i s$ & $17(19,54 \%)$ & $2(15,38 \%)$ & \\
\hline 4 Lupusnephritis & $6(6,90 \%)$ & $1(7,69 \%)$ & \\
\hline 5 membranoproliferative GN & $2(2,30 \%)$ & $0(0,00 \%)$ & \\
\hline 6 membranöse GN & $3(3,45 \%)$ & $0(0,00 \%)$ & \\
\hline 7 Transplantat - Glomerulopathie & $4(4,60 \%)$ & $0(0,00 \%)$ & \\
\hline 8 minimal change GN & $4(4,60 \%)$ & $0(0,00 \%)$ & \\
\hline 9 diabetische Nephropathie & $2(2,30 \%)$ & $0(0,00 \%)$ & \\
\hline 10 Andere & $19(21,84 \%)$ & $4(30,77 \%)$ & \\
\hline
\end{tabular}

Tab. 3 


\begin{tabular}{|c|c|c|c|}
\hline Parameter & Keine Komplikation & Makrohämaturie & p-Wert \\
\hline PFA & & & 0,2578 \\
\hline 1 zu niedrig & $10(10,53 \%)$ & $0(0,00 \%)$ & \\
\hline 2 normal & $61(64,21 \%)$ & $5(100,00 \%)$ & \\
\hline 3 zu hoch & $24(25,26 \%)$ & $0(0,00 \%)$ & \\
\hline Geschlecht & & & 0,4028 \\
\hline 1 männlich & $56(58,95 \%)$ & $2(40,00 \%)$ & \\
\hline 2 weiblich & $39(41,05 \%)$ & $3(60,00 \%)$ & \\
\hline Nadeldicke & & & 0,7799 \\
\hline $114 G$ & $42(51,22 \%)$ & $3(60,00 \%)$ & \\
\hline $216 G$ & $33(40,24 \%)$ & $2(40,00 \%)$ & \\
\hline $318 G$ & $7(8,54 \%)$ & $0(0,00 \%)$ & \\
\hline Quick & & & 0,8845 \\
\hline $1<70 \%$ & $3(3,30 \%)$ & $0(0,00 \%)$ & \\
\hline $270-120 \%$ & $58(63,74 \%)$ & $3(60,00 \%)$ & \\
\hline $3>120 \%$ & $30(32,97 \%)$ & $2(40,00 \%)$ & \\
\hline MDRD & & & 0,3013 \\
\hline $1>60 \mathrm{ml} / \mathrm{min} / 1,73 \mathrm{~m}^{2} \mathrm{KO}$ & $25(32,47 \%)$ & $0(0,00 \%)$ & \\
\hline $230-60 \mathrm{ml} / \mathrm{min} / 1,73 \mathrm{~m}^{2} \mathrm{KO}$ & $21(27,27 \%)$ & $1(25,00 \%)$ & \\
\hline $3<30 \mathrm{ml} / \mathrm{min} / 1,73 \mathrm{~m}^{2} \mathrm{KO}$ & $31(40,26 \%)$ & $3(75,00 \%)$ & \\
\hline Arterielle Hypertonie & & & 0,3949 \\
\hline $1 \mathrm{ja}$ & $58(61,05 \%)$ & $4(80,00 \%)$ & \\
\hline 2 nein & $37(38,95 \%)$ & $1(20,00 \%)$ & \\
\hline Diabetes mellitus & & & 0,2992 \\
\hline $1 \mathrm{ja}$ & $17(17,89 \%)$ & $0(0,00 \%)$ & \\
\hline 2 nein & $78(82,11 \%)$ & $5(200,00 \%)$ & \\
\hline Transplantatniere & & & 0,3188 \\
\hline 1 ja & $20(21,05 \%)$ & $2(40,00 \%)$ & \\
\hline 2 nein & $75(78,95 \%)$ & $3(60,00 \%)$ & \\
\hline Nephrologische Diagnose & & & 0,8786 \\
\hline 1 Nephrosklerose & $15(15,79 \%)$ & $2(40,00 \%)$ & \\
\hline 2 FSGS & $19(20,00 \%)$ & $0(0,00 \%)$ & \\
\hline $3 \lg A-N e p h r i t i s$ & $18(18,95 \%)$ & $1(20,00 \%)$ & \\
\hline 4 Lupusnephritis & $7(7,37 \%)$ & $0(0,00 \%)$ & \\
\hline 5 membranoproliferative GN & $2(2,11 \%)$ & $0(0,00 \%)$ & \\
\hline 6 membranöse GN & $3(3,16 \%)$ & $0(0,00 \%)$ & \\
\hline 7 Transplantat - Glomerulopathie & $4(4,21 \%)$ & $0(0,00 \%)$ & \\
\hline 8 minimal change GN & $4(4,21 \%)$ & $0(0,00 \%)$ & \\
\hline 9 diabetische Nephropathie & $2(2,11 \%)$ & $0(0,00 \%)$ & \\
\hline 10 Andere & $21(22,11 \%)$ & $2(40,00 \%)$ & \\
\hline
\end{tabular}

Tab. 4 


\begin{tabular}{|c|c|c|c|}
\hline Parameter & Keine Komplikation & Blutung & p-Wert \\
\hline PFA & & & 0,3173 \\
\hline 1 zu niedrig & $9(9,28 \%)$ & $1(33,33 \%)$ & \\
\hline 2 normal & $65(67,01 \%)$ & $1(33,33 \%)$ & \\
\hline 3 zu hoch & $23(23,71 \%)$ & $1(33,33 \%)$ & \\
\hline Geschlecht & & & 0,7575 \\
\hline 1 männlich & $56(57,73 \%)$ & $2(66,67 \%)$ & \\
\hline 2 weiblich & $41(42,27 \%)$ & $1(33,33 \%)$ & \\
\hline Nadeldicke & & & 0,6087 \\
\hline $114 G$ & $44(52,38 \%)$ & $1(33,33 \%)$ & \\
\hline $216 G$ & $33(39,29 \%)$ & $2(66,67 \%)$ & \\
\hline $318 G$ & $7(8,33 \%)$ & $0(0,00 \%)$ & \\
\hline Quick & & & 0,5566 \\
\hline $1<70 \%$ & $3(3,19 \%)$ & $0(0,00 \%)$ & \\
\hline $270-120 \%$ & $59(62,77 \%)$ & $2(100,00 \%)$ & \\
\hline $3>120 \%$ & $32(34,04 \%)$ & $0(0,00 \%)$ & \\
\hline MDRD & & & 0,2424 \\
\hline $1>60 \mathrm{ml} / \mathrm{min} / 1,73 \mathrm{~m}^{2} \mathrm{KO}$ & $25(31,65 \%)$ & $0(0,00 \%)$ & \\
\hline $230-60 \mathrm{ml} / \mathrm{min} / 1,73 \mathrm{~m}^{2} \mathrm{KO}$ & $22(27,85 \%)$ & $0(0,00 \%)$ & \\
\hline $3<30 \mathrm{ml} / \mathrm{min} / 1,73 \mathrm{~m}^{2} \mathrm{KO}$ & $32(40,51 \%)$ & $2(100,00 \%)$ & \\
\hline Arterielle Hypertonie & & & 0,2990 \\
\hline 1 ja & $61(62,89 \%)$ & $1(33,33 \%)$ & \\
\hline 2 nein & $36(37,11 \%)$ & $2(66,67 \%)$ & \\
\hline Diabetes mellitus & & & 0,4261 \\
\hline $1 \mathrm{ja}$ & $17(17,53 \%)$ & $0(0,00 \%)$ & \\
\hline 2 nein & $80(82,47 \%)$ & $3(100,00 \%)$ & \\
\hline Transplantatniere & & & 0,3503 \\
\hline $1 \mathrm{ja}$ & $22(22,68 \%)$ & $0(0,00 \%)$ & \\
\hline 2 nein & $75(77,32 \%)$ & $3(100,00 \%)$ & \\
\hline Nephrologische Diagnose & & & 0,7747 \\
\hline 1 Nephrosklerose & $16(16,49 \%)$ & $1(33,33 \%)$ & \\
\hline 2 FSGS & $18(18,56 \%)$ & $1(33,33 \%)$ & \\
\hline $3 \lg A-N e p h r i t i s$ & $19(19,59 \%)$ & $0(0,00 \%)$ & \\
\hline 4 Lupusnephritis & $6(6,19 \%)$ & $1(33,33 \%)$ & \\
\hline 5 membranoproliferative GN & $2(2,06 \%)$ & $0(0,00 \%)$ & \\
\hline 6 membranöse GN & $3(3,09 \%)$ & $0(0,00 \%)$ & \\
\hline 7 Transplantat - Glomerulopathie & $4(4,12 \%)$ & $0(0,00 \%)$ & \\
\hline 8 minimal change GN & $4(4,12 \%)$ & $0(0,00 \%)$ & \\
\hline 9 diabetische Nephropathie & $2(2,06 \%)$ & $0(0,00 \%)$ & \\
\hline 10 Andere & $23(23,71 \%)$ & $0(0,00 \%)$ & \\
\hline
\end{tabular}

Tab. 5 


\begin{tabular}{|c|c|c|c|}
\hline Parameter & Keine Komplikation & $\begin{array}{l}\text { Klinisch relevanter } \\
\text { Hb-Abfall }\end{array}$ & p-Wert \\
\hline PFA & & & 0,1418 \\
\hline 1 zu niedrig & $10(10,53 \%)$ & $0(0,00 \%)$ & \\
\hline 2 normal & $64(67,37 \%)$ & $2(40,00 \%)$ & \\
\hline 3 zu hoch & $21(22,11 \%)$ & $3(60,00 \%)$ & \\
\hline Geschlecht & & & 0,0509 \\
\hline 1 männlich & $53(55,79 \%)$ & $5(100,00 \%)$ & \\
\hline 2 weiblich & $42(44,21 \%)$ & $0(0,00 \%)$ & \\
\hline Nadeldicke & & & 0,5818 \\
\hline $114 G$ & $43(52,44 \%)$ & $2(40,00 \%)$ & \\
\hline $216 G$ & $32(39,02 \%)$ & $3(60,00 \%)$ & \\
\hline $318 \mathrm{G}$ & $7(8,54 \%)$ & $0(0,00 \%)$ & \\
\hline Quick & & & 0,4168 \\
\hline $1<70 \%$ & $3(3,30 \%)$ & $0(0,00 \%)$ & \\
\hline $270-120 \%$ & $59(64,84 \%)$ & $2(40,00 \%)$ & \\
\hline $3>120 \%$ & $29(31,87 \%)$ & $3(60,00 \%)$ & \\
\hline MDRD & & & 0,3193 \\
\hline $1>60 \mathrm{ml} / \mathrm{min} / 1,73 \mathrm{~m}^{2} \mathrm{KO}$ & $24(31,17 \%)$ & $1(25,00 \%)$ & \\
\hline $230-60 \mathrm{ml} / \mathrm{min} / 1,73 \mathrm{~m}^{2} \mathrm{KO}$ & $22(28,57 \%)$ & $0(0,00 \%)$ & \\
\hline $3<30 \mathrm{ml} / \mathrm{min} / 1,73 \mathrm{~m}^{2} \mathrm{KO}$ & $31(40,26 \%)$ & $3(75,00 \%)$ & \\
\hline Arterielle Hypertonie & & & 0,3949 \\
\hline $1 \mathrm{ja}$ & $58(61,05 \%)$ & $4(80,00 \%)$ & \\
\hline 2 nein & $37(38,95 \%)$ & $1(20,00 \%)$ & \\
\hline Diabetes mellitus & & & 0,1601 \\
\hline $1 \mathrm{ja}$ & $15(15,79 \%)$ & $2(40,00 \%)$ & \\
\hline 2 nein & $80(84,21 \%)$ & $3(60,00 \%)$ & \\
\hline Transplantatniere & & & 0,9118 \\
\hline $1 \mathrm{ja}$ & $21(22,11 \%)$ & $1(20,00 \%)$ & \\
\hline 2 nein & $74(77,89 \%)$ & $4(80,00 \%)$ & \\
\hline Nephrologische Diagnose & & & 0,8786 \\
\hline 1 Nephrosklerose & $15(15,79 \%)$ & $2(40,00 \%)$ & \\
\hline 2 FSGS & $19(20,00 \%)$ & $0(0,00 \%)$ & \\
\hline $3 \lg A-N e p h r i t i s$ & $18(18,95 \%)$ & $1(20,00 \%)$ & \\
\hline 4 Lupusnephritis & $7(7,37 \%)$ & $0(0,00 \%)$ & \\
\hline 5 membranoproliferative GN & $2(2,11 \%)$ & $0(0,00 \%)$ & \\
\hline 6 membranöse GN & $3(3,16 \%)$ & $0(0,00 \%)$ & \\
\hline 7 Transplantat - Glomerulopathie & $4(4,21 \%)$ & $0(0,00 \%)$ & \\
\hline 8 minimal change GN & $4(4,21 \%)$ & $0(0,00 \%)$ & \\
\hline 9 diabetische Nephropathie & $2(2,11 \%)$ & $0(0,00 \%)$ & \\
\hline 10 Andere & $21(22,11 \%)$ & $2(40,00 \%)$ & \\
\hline
\end{tabular}

Tab. 6 


\begin{tabular}{|l|lllll|l|}
\hline Parameter & \multicolumn{2}{|l|}{ Kein Hämatom } & & Hämatom & p-Wert \\
& MW +/- SF & $\mathbf{( N )}$ & MW +/- SF & (N) & \\
\hline PFA & $145,36+/-9,97$ & $(55)$ & $154,11+/-19,97$ & $(19)$ & 0,6726 \\
\hline Alter & $52,24+/-1,99$ & $(55)$ & $44,42+/-3,61$ & $(19)$ & 0,0545 \\
\hline Stat. Aufenthalt & $14,89+/-2,32$ & $(53)$ & $13,44+/-3,78$ & $(18)$ & 0,7526 \\
\hline Hämoglobin & $12,28+/-0,25$ & $(54)$ & $12,42+/-0,45$ & $(19)$ & 0,7895 \\
\hline Quick & $111,94+/-1,94$ & $(52)$ & $107,16+/-5,89$ & $(19)$ & 0,3198 \\
\hline PTT & $27,52+/-0,42$ & $(54)$ & $29,06+/-1,76$ & $(19)$ & 0,2224 \\
\hline Kreatinin & $2,02+/-0,19$ & $(55)$ & $2,66+/-0,53$ & $(19)$ & 0,1631 \\
\hline Kreatininclearance & $63,19+/-6,46$ & $(34)$ & $67,32+/-16,74$ & $(11)$ & 0,7810 \\
\hline MDRD & $40,93+/-2,56$ & $(43)$ & $40,09+/-4,91$ & $(14)$ & 0,8743 \\
\hline
\end{tabular}

Tab. 7

\begin{tabular}{|l|lll|ll|l|}
\hline Parameter & \multicolumn{3}{|l|}{ Kein Hb-Abfall } & & Hb-Abfall & p-Wert \\
& MW +/- SF & $\mathbf{( N )}$ & MW +/- SF & (N) & \\
\hline PFA & $135,89+/-9,13$ & $(55)$ & $164,41+/-12,42$ & $(41)$ & 0,0613 \\
\hline Alter & $50,67+/-2,04$ & $(55)$ & $48,90+/-2,35$ & $(41)$ & 0,5707 \\
\hline Stat. Aufenthalt & $14,43+/-2,38$ & $(54)$ & $14,13+/-2,22$ & $(39)$ & 0,9299 \\
\hline Hämoglobin & $12,17+/-0,23$ & $(55)$ & $12,03+/-0,31$ & $(41)$ & 0,7273 \\
\hline Quick & $112,77+/-2,14$ & $(53)$ & $107,05+/-3,24$ & $(39)$ & 0,1289 \\
\hline PTT & $27,82+/-0,56$ & $(54)$ & $28,28+/-0,86$ & $(40)$ & 0,6427 \\
\hline Kreatinin & $2,27+/-0,22$ & $(55)$ & $2,53+/-0,34$ & $(41)$ & 0,5075 \\
\hline Kreatininclearance & $56,56+/-6,81$ & $(36)$ & $57,51+/-8,48$ & $(22)$ & 0,9313 \\
\hline MDRD & $40,14+/-2,52$ & $(45)$ & $38,48+/-2,99$ & $(33)$ & 0,6724 \\
\hline
\end{tabular}

Tab. 8

\begin{tabular}{|l|ll|ll|l|}
\hline Parameter & \multicolumn{2}{|l|}{ Keine Komplikation } & \multicolumn{2}{|l|}{ Komplikation } & \multicolumn{2}{l|}{ p-Wert } \\
& MW +/- SF & $\mathbf{( N )}$ & MW +/- SF & (N) & \\
\hline PFA & $144,93+/-7,83$ & $(87)$ & $160,85+/-20,13$ & $(13)$ & 0,4649 \\
\hline Alter & $49,18+/-1,70$ & $(87)$ & $52,15+/-2,93$ & $(13)$ & 0,5166 \\
\hline Stat. Aufenthalt & $14,14+/-1,76$ & $(85)$ & $14,92+/-3,26$ & $(12)$ & 0,8736 \\
\hline Hämoglobin & $12,28+/-0,19$ & $(84)$ & $11,02+/-0,53$ & $(13)$ & $\mathbf{0 , 0 1 9 5}$ \\
\hline Quick & $109,67+/-2,02$ & $(84)$ & $15,33+/-3,72$ & $(12)$ & 0,3092 \\
\hline PTT & $28,26+/-0,51$ & $(85)$ & $26,17+/-0,93$ & $(13)$ & 0,1305 \\
\hline Kreatinin & $2,02+/-0,14$ & $(87)$ & $4,59+/-0,81$ & $(13)$ & $<0,0001$ \\
\hline Kreatininclearance & $61,47+/-5,45$ & $(53)$ & $36,09+/-14,58$ & $(8)$ & 0,0986 \\
\hline MDRD & $41,27+/-1,97$ & $(71)$ & $26,63+/-4,12$ & $(10)$ & $\mathbf{0 , 0 0 9 2}$ \\
\hline
\end{tabular}

Tab. 9 


\begin{tabular}{|l|ll|ll|l|}
\hline Parameter & \multicolumn{2}{|l|}{ Keine Komplikation } & \multicolumn{2}{|l|}{ Makrohämaturie } & p-Wert \\
& MW +/- SF & $\mathbf{( N )}$ & MW +/- SF & (N) & \\
\hline PFA & $144,93+/-7,83$ & $(87)$ & $127,60+/-12,20$ & $(5)$ & 0,5999 \\
\hline Alter & $49,18+/-1,70$ & $(87)$ & $52,00+/-3,11$ & $(5)$ & 0,6958 \\
\hline Stat. Aufenthalt & $14,14+/-1,76$ & $(85)$ & $13,75+/-3,94$ & $(4)$ & 0,9621 \\
\hline Hämoglobin & $12,28+/-0,19$ & $(84)$ & $12,40+/-1,08$ & $(5)$ & 0,8838 \\
\hline Quick & $109,67+/-2,02$ & $(84)$ & $117,60+/-4,12$ & $(5)$ & 0,3464 \\
\hline PTT & $28,26+/-0,51$ & $(85)$ & $24,20+/-1,02$ & $(5)$ & 0,0620 \\
\hline Kreatinin & $2,02+/-0,14$ & $(87)$ & $4,08+/-1,12$ & $(5)$ & $\mathbf{0 , 0 0 2 1}$ \\
\hline Kreatininclearance & $61,47+/-5,45$ & $(53)$ & $36,15+/-17,75$ & $(2)$ & 0,3771 \\
\hline MDRD & $41,27+/-1,97$ & $(71)$ & $24,08+/-4,08$ & $(4)$ & $\mathbf{0 , 0 4 4 3}$ \\
\hline
\end{tabular}

Tab. 10

\begin{tabular}{|c|c|c|c|c|c|}
\hline \multirow[t]{2}{*}{ Parameter } & \multicolumn{2}{|c|}{ Keine Komplikation } & \multicolumn{2}{|l|}{ Blutung } & \multirow[t]{2}{*}{ p-Wert } \\
\hline & MW +/- SF & (N) & $\mathrm{MW}+/-\mathrm{SF}$ & (N) & \\
\hline PFA & $144,93+/-7,83$ & (87) & $162,67+/-70,68$ & (3) & 0,6861 \\
\hline Alter & $49,24+/-1,70$ & (87) & $60,33+/-8,37$ & (3) & 0,2178 \\
\hline Stat. Aufenthalt & $14,59+/-1,76$ & (85) & $3,33+/-0,32$ & (3) & 0,2232 \\
\hline Hämoglobin & $12,16+/-0,19$ & $(84)$ & $10,40+/-0,64$ & (3) & 0,0999 \\
\hline Quick & $109,67+/-2,02$ & (84) & $98,00+/-1,00$ & (2) & 0,3785 \\
\hline PTT & $27,95+/-0,51$ & (85) & $29,00+/-3,06$ & (3) & 0,6997 \\
\hline Kreatinin & $2,23+/-0,14$ & (87) & $6,33+/-1,67$ & (3) & 0,0001 \\
\hline Kreatininclearance & $59,80+/-5,45$ & (53) & $9,25+/-5,75$ & (2) & 0,0823 \\
\hline MDRD & $41,27+/-1,97$ & $(71)$ & $20,00+/-0,00$ & $(2)$ & 0,0763 \\
\hline
\end{tabular}

Tab. 11

\begin{tabular}{|c|c|c|c|c|c|}
\hline \multirow[t]{2}{*}{ Parameter } & \multicolumn{2}{|c|}{ Keine Komplikation } & \multicolumn{2}{|c|}{$\begin{array}{l}\text { Klinisch relevanter } \\
\text { Hb-Abfall }\end{array}$} & \multirow[t]{2}{*}{ p-Wert } \\
\hline & $\mathrm{MW}$ +/- SF & (N) & $\begin{array}{l}\text { Hb-Abfall } \\
M W+/-S F\end{array}$ & & \\
\hline PFA & $144,93+/-7,83$ & $(87)$ & $193,00+/-31,27$ & $(5)$ & 0,1549 \\
\hline Alter & $49,18+/-1,70$ & $(87)$ & $47,40+/-4,53$ & (5) & 0,8053 \\
\hline Stat. Aufenthalt & $14,14+/-1,76$ & (85) & $22,80+/-4,97$ & (5) & 0,2447 \\
\hline Hämoglobin & $12,28+/-0,19$ & (84) & $10,00+/-0,35$ & (5) & 0,0053 \\
\hline Quick & $109,67+/-2,02$ & $(84)$ & $120,00+/-6,13$ & (5) & 0,2241 \\
\hline PTT & $28,26+/-0,51$ & (85) & $26,44+/-0,83$ & (5) & 0,3988 \\
\hline Kreatinin & $2,02+/-0,1487$ & $(87)$ & $4,06+/-1,55$ & (5) & 0,0040 \\
\hline Kreatininclearance & $61,47+/-5,45$ & (53) & $49,48+/-27,46$ & (4) & 0,5718 \\
\hline MDRD & $41,27+/-1,97$ & (71) & $32,50+/-9,46$ & (4) & 0,3106 \\
\hline
\end{tabular}

Tab. 12 


\subsection{PFA-100 ${ }^{\mathrm{TM}}$ als Parameter zur Evaluation von Risiken}

\subsubsection{Berechnung mit ordinalskalierten Werten}

Wie bereits beschrieben, wurden zunächst alle Werte $>300 \mathrm{sec}$ mit $300 \mathrm{sec}$ gleichgesetzt, da sie nicht exakt angegeben werden konnten. Mit dieser Berechnung ergab sich für PFA-100 ${ }^{\mathrm{TM}}$ ein Mittelwert von $147 \mathrm{sec}$ mit einem Minimum bei $57 \mathrm{sec}$ und einem Maximum bei $300 \mathrm{sec}$. Der Median lag bei 120,5 sec.

Durchgeführt wurde jeweils der t-Test für unabhängige Stichproben.

\subsubsection{Beziehung zwischen PFA- $100^{\mathrm{TM}}$ und Hämatom}

Die Patienten mit Hämatomentwicklung nach der Biopsie wiesen keinen signifikanten Unterschied in der Höhe des PFA-100 ${ }^{\mathrm{TM}}$-Spiegels zu den Patienten, die kein Hämatom entwickelten ( $p$ 0,6726).

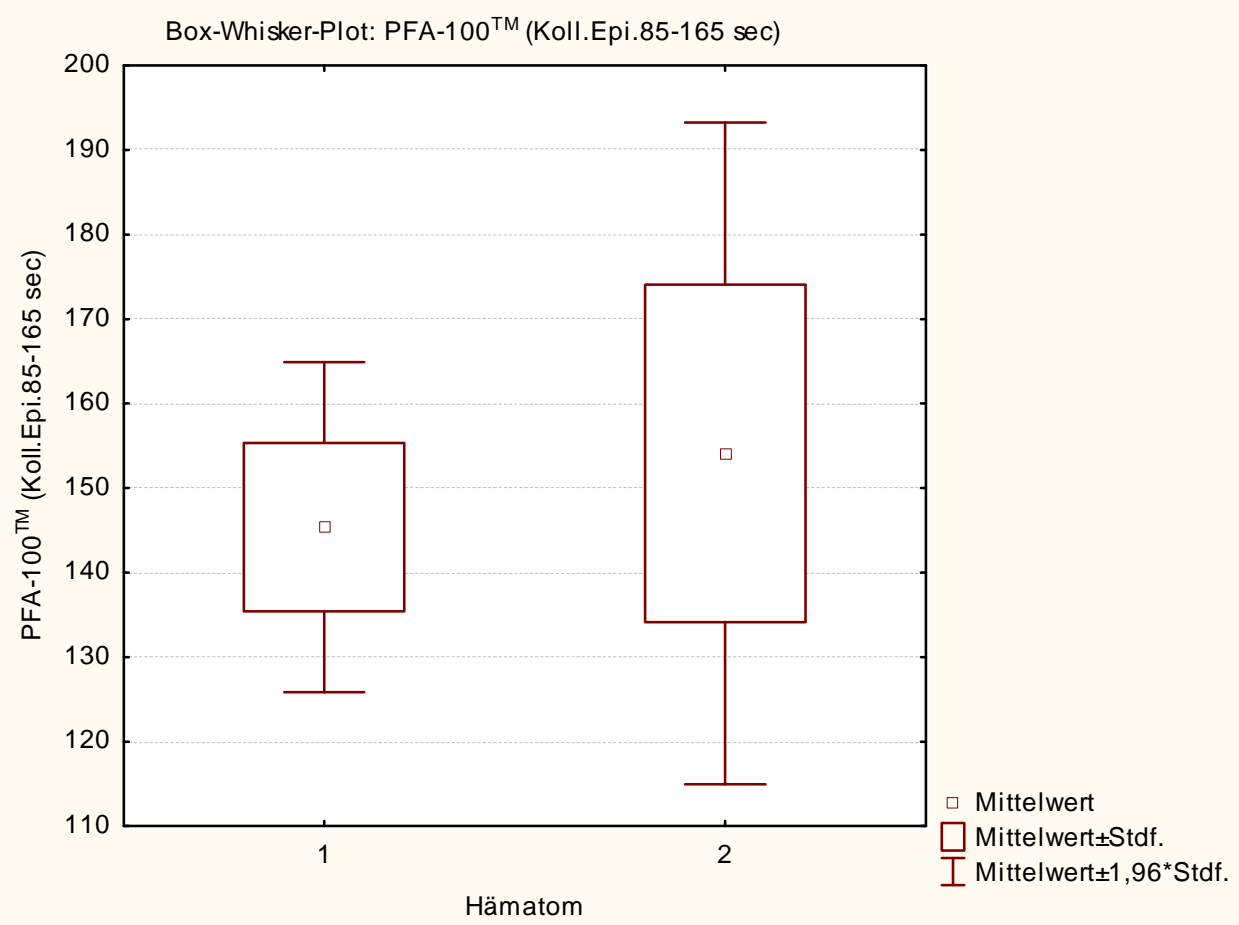

Abb. 1: Beziehung zwischen PFA-100 ${ }^{\mathrm{TM}}$ und Hämatom.

$1=$ kein Hämatom, 2 = Hämatom 


\subsubsection{Beziehung zwischen PFA- $100^{\mathrm{TM}}$ und $\mathrm{Hb}-$ Abfall $>1 \mathrm{~g} / \mathrm{dl}$}

Die Patienten mit einem $\mathrm{Hb}$-Abfall $>1 \mathrm{~g} / \mathrm{dl}$ in den ersten 24 Stunden nach Biopsie zeigten keinen signifikanten Unterschied in der Höhe des PFA-100 ${ }^{\mathrm{TM}}$ Spiegels zu

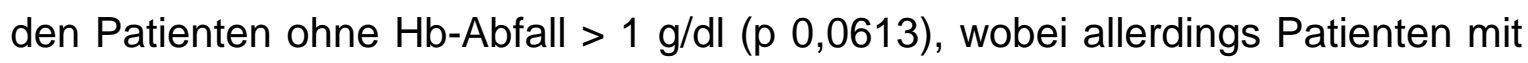
einem höheren PFA-100 ${ }^{\mathrm{TM}}$-Wert tendenziell häufiger einen $\mathrm{Hb}$-Abfall $>1 \mathrm{~g} / \mathrm{dl}$ aufwiesen.

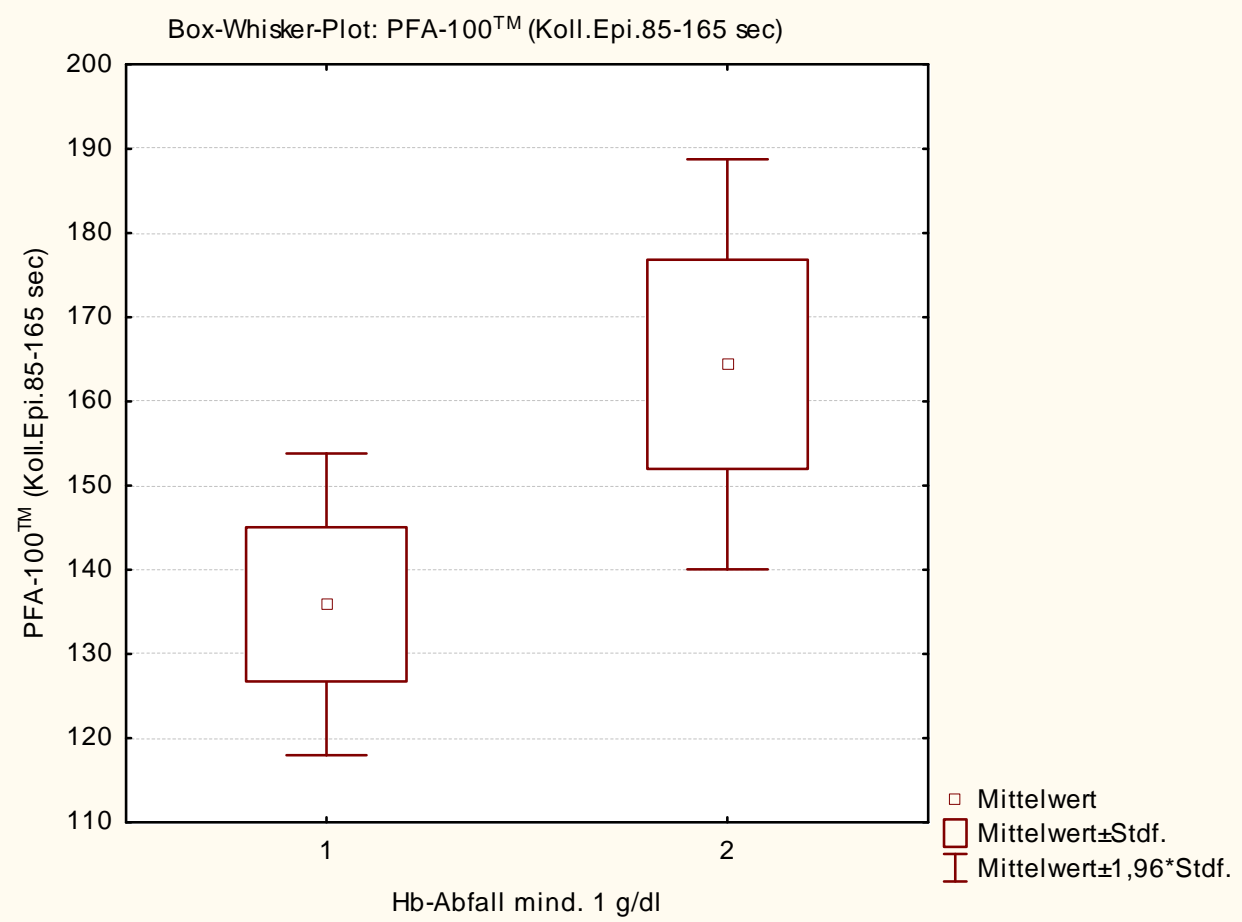

Abb. 2: Beziehung zwischen PFA- $100^{\mathrm{TM}}$ und $\mathrm{Hb}-$ Abfall $>1 \mathrm{~g} / \mathrm{dl}$.

$1=$ kein $\mathrm{Hb}$-Abfall, $2=\mathrm{Hb}$-Abfall

\subsubsection{Beziehung zwischen PFA-100 ${ }^{\mathrm{TM}}$ und Komplikation}

Die Patienten mit einer Komplikation nach Biopsie unterschieden sich in der Höhe des PFA-100 ${ }^{\text {TM }}$-Spiegels nicht signifikant von den Patienten ohne Komplikation ( $\mathrm{p}$ $0,4649)$. 


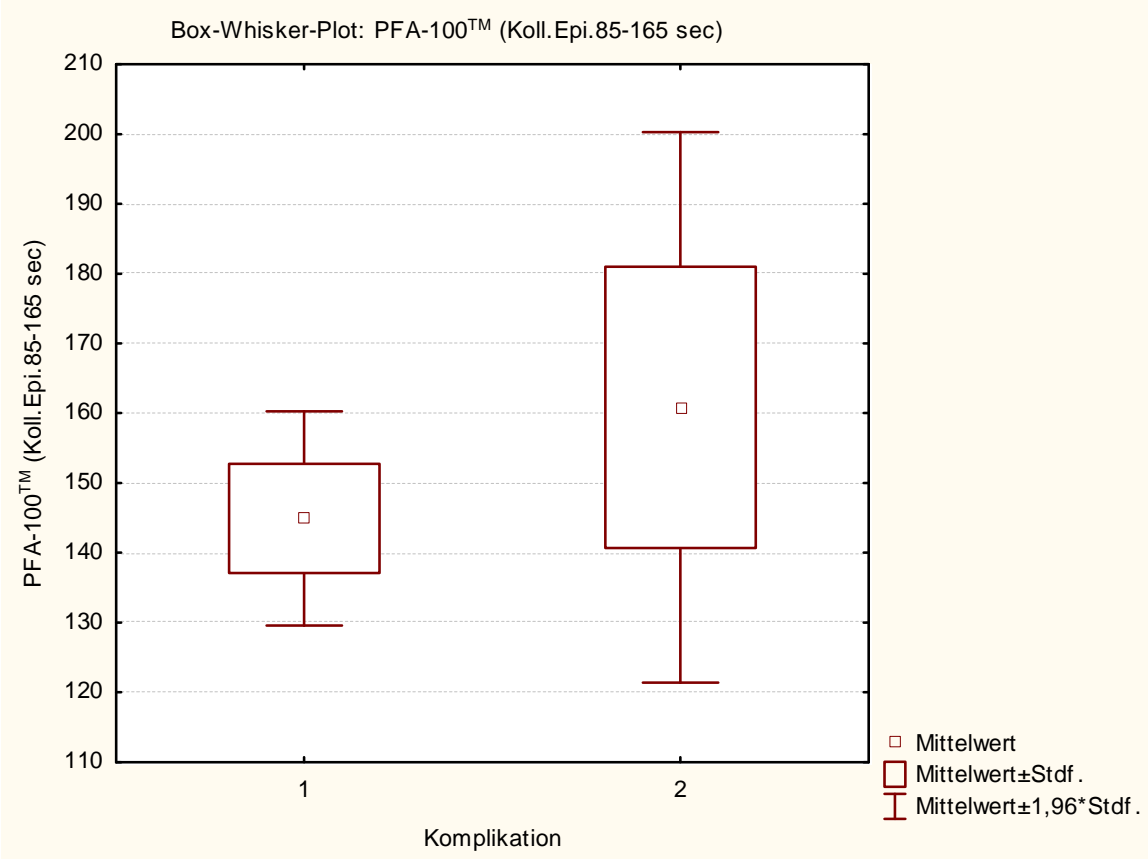

Abb. 3: Beziehung zwischen PFA-100 ${ }^{\mathrm{TM}}$ und Komplikation.

$1=$ keine Komplikation, $2=$ Komplikation

\subsubsection{Beziehung zwischen PFA- $100^{\mathrm{TM}}$ und Komplikationsart}

Die Komplikationsart wurde in drei Gruppen unterteilt:

1) Makrohämaturie

Die Höhe des PFA-100 ${ }^{\mathrm{TM}}$-Spiegels war nicht signifikant unterschiedlich zwischen den Patienten mit einer neu aufgetretenen Makrohämaturie und den Patienten ohne Komplikation ( $p$ 0,5999).

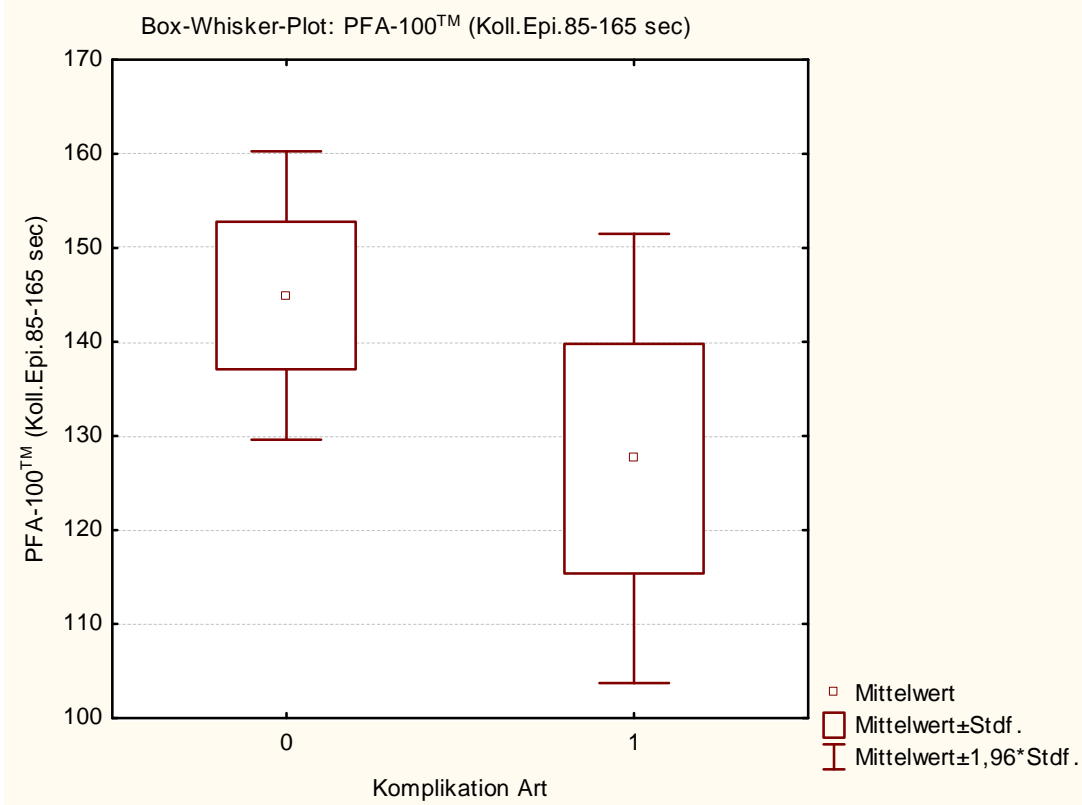

Abb. 4: Beziehung zwischen PFA-100 ${ }^{\mathrm{TM}}$ und Komplikationsart $\rightarrow$ Makrohämaturie.

$0=$ keine Komplikation, $1=$ Makrohämaturie 


\section{2) Blutung}

Die Patienten mit einer Blutung nach Biopsie wiesen keinen signifikanten Unterschied in der Höhe des PFA-100 10 -Spiegels auf zu den Patienten ohne Komplikation (p 0,6861).

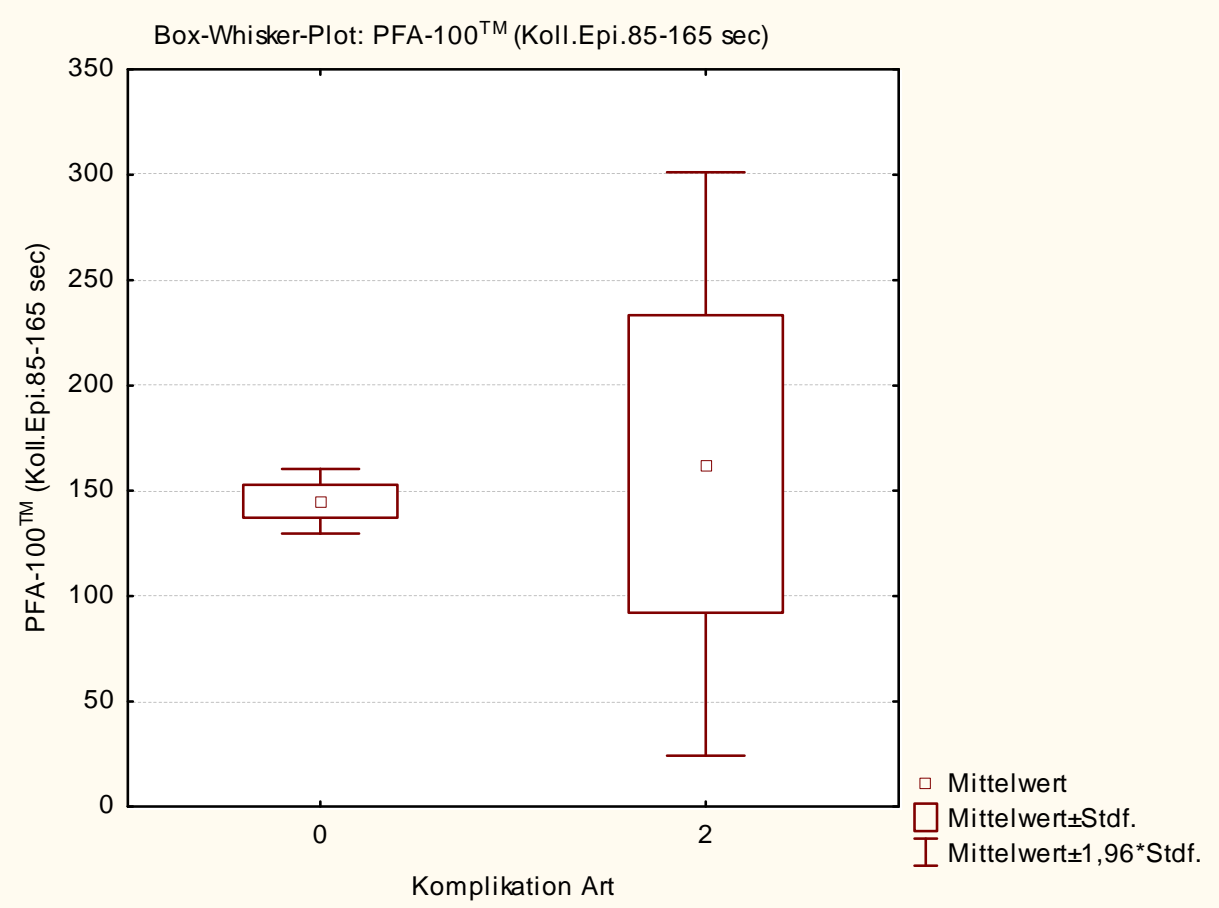

Abb. 5: Beziehung zwischen PFA-100 ${ }^{\mathrm{TM}}$ und Komplikationsart $\rightarrow$ Blutung.

$0=$ keine Komplikation, 2 = Blutung 
3) Klinisch relevanter Hb-Abfall

Es ergab sich kein signifikanter Unterschied in der Höhe des PFA-100 ${ }^{\mathrm{TM}}$ Spiegels bei dem Vergleich der Patienten mit und ohne klinisch relevanten $\mathrm{Hb}$ Abfall ( $p$ 0,1549).

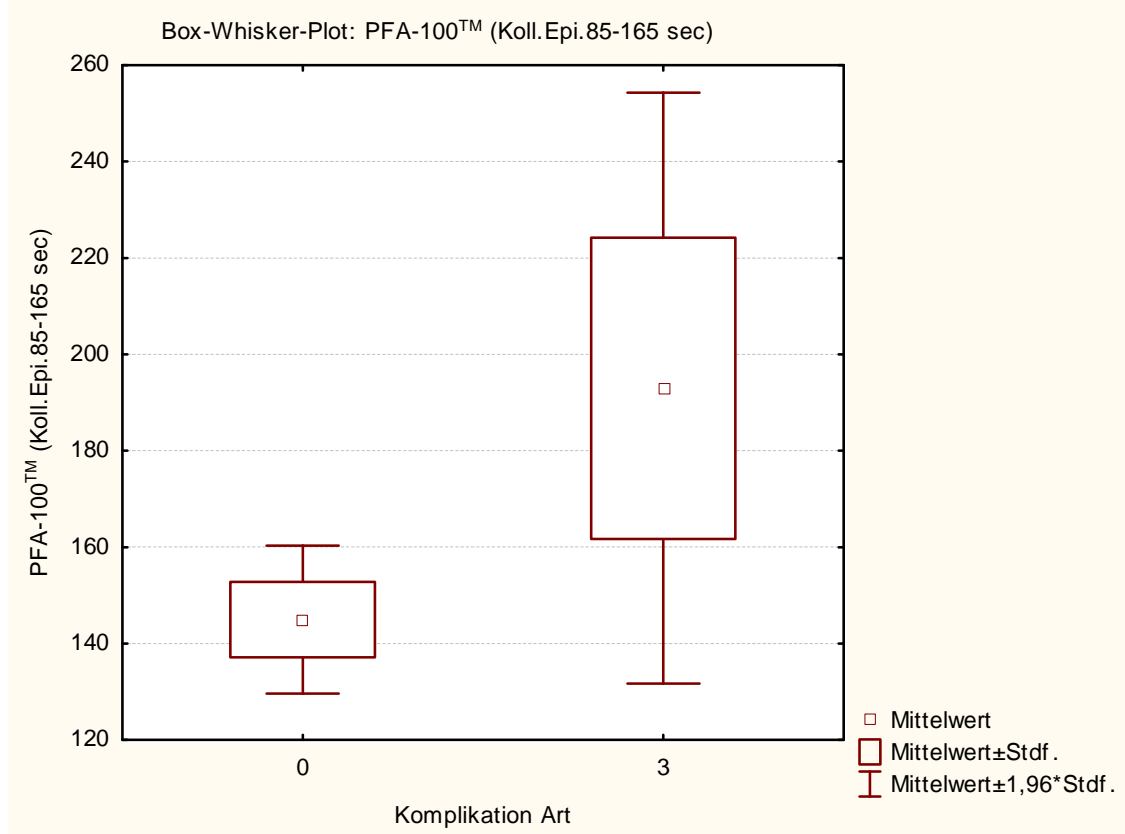

Abb. 6: Beziehung zwischen PFA-100 ${ }^{\mathrm{TM}}$ und Komplikationsart $\rightarrow \mathrm{Hb}$-Abfall.

$0=$ keine Komplikation, $3=\mathrm{Hb}$-Abfall

\subsubsection{Berechnung mit nominalskalierten Werten}

Aus den bereits angeführten Gründen erfolgte nun eine zweite Berechnung. Dazu wurden die Patienten in verschiedene Gruppen unterteilt: 1: PFA $<85 \mathrm{sec}, 2$ : PFA 85 - 165 sec, 3: PFA > 165 sec.

Durchgeführt wurde jeweils der Chi-Quadrat-Test nach Pearson. 


\subsubsection{Beziehung zwischen PFA-100 $100^{\mathrm{TM}}$ und Hämatom}

Die Patienten mit Hämatomentwicklung zeigten keinen signifikanten Unterschied in der prozentualen Verteilung auf die PFA-Gruppen zu den Patienten ohne Hämatom ( $p$ 0,1024).

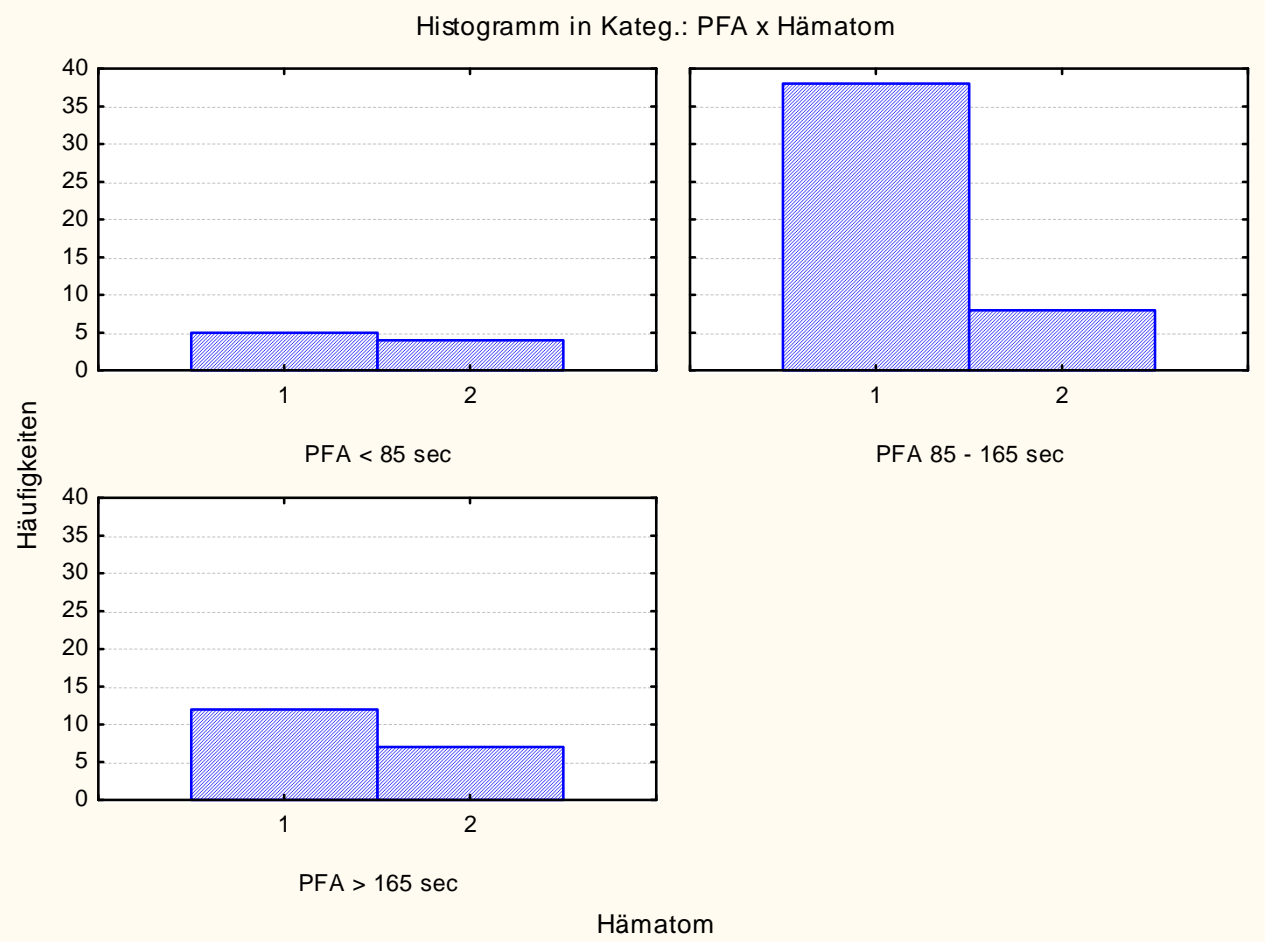

Abb. 7: Beziehung zwischen PFA- $100^{\mathrm{TM}}$ und Hämatom.

1 = kein Hämatom, 2 = Hämatom

3.3.2.2 Beziehung zwischen PFA-100 ${ }^{\mathrm{TM}}$ und $\mathrm{Hb}-$ Abfall $>1 \mathrm{~g} / \mathrm{dl}$

Die Patienten mit $\mathrm{Hb}$-Abfall > $1 \mathrm{~g} / \mathrm{dl}$ innerhalb der ersten 24 Stunden nach Biopsie unterschieden sich in der prozentualen Verteilung auf die PFA-Gruppen nicht signifikant von den Patienten ohne Hb-Abfall > $1 \mathrm{~g} / \mathrm{dl}(\mathrm{p} \mathrm{0,1773).}$ 


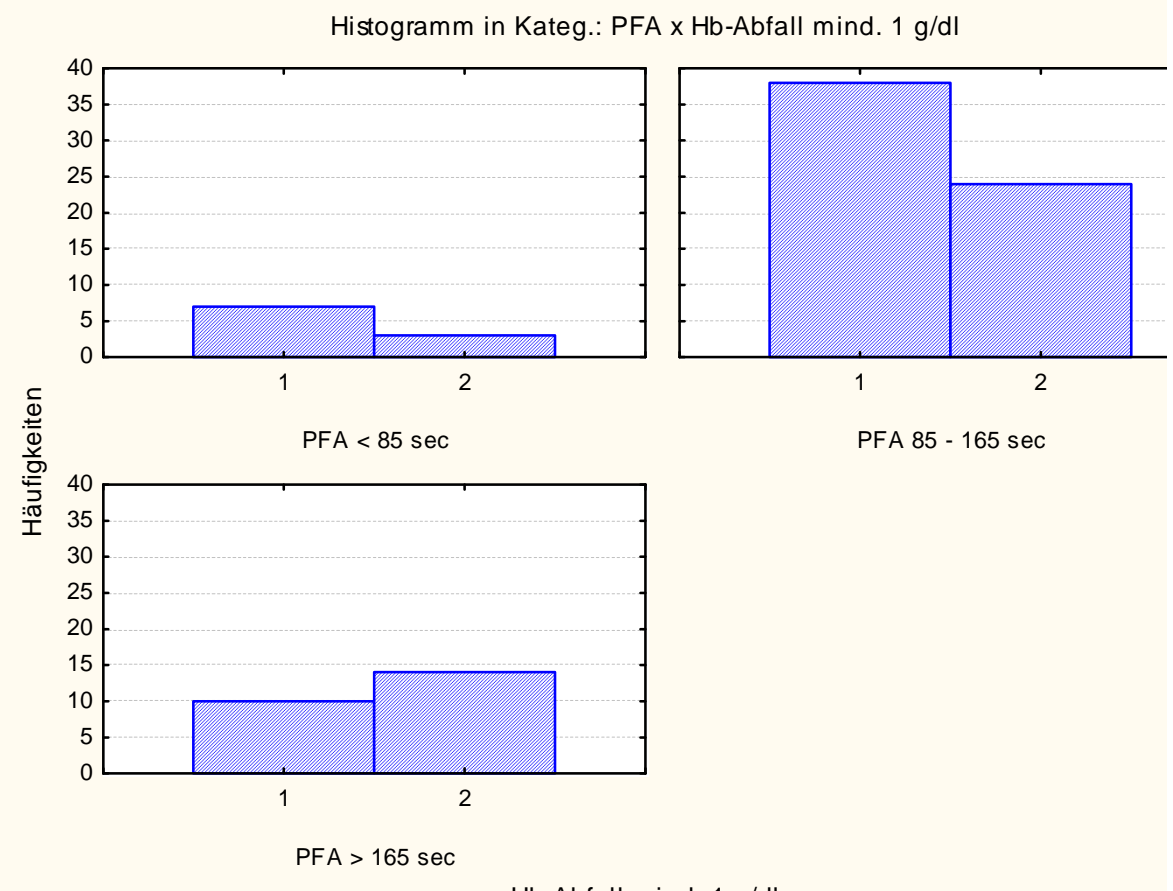

Hb-Abfall mind. $1 \mathrm{~g} / \mathrm{dl}$

Abb. 8: Beziehung zwischen PFA- $100^{\mathrm{TM}}$ und $\mathrm{Hb}-$ Abfall mind. $1 \mathrm{~g} / \mathrm{dl}$. $1=$ kein $\mathrm{Hb}-$ Abfall, $2=\mathrm{Hb}$-Abfall

\subsubsection{Beziehung zwischen PFA-100 ${ }^{\mathrm{TM}}$ und Komplikation}

Die Patienten mit einer Komplikation nach Biopsie unterschieden sich nicht signifikant in der prozentualen Verteilung auf die PFA-Gruppen von den Patienten ohne Komplikation ( $p$ 0,8147).

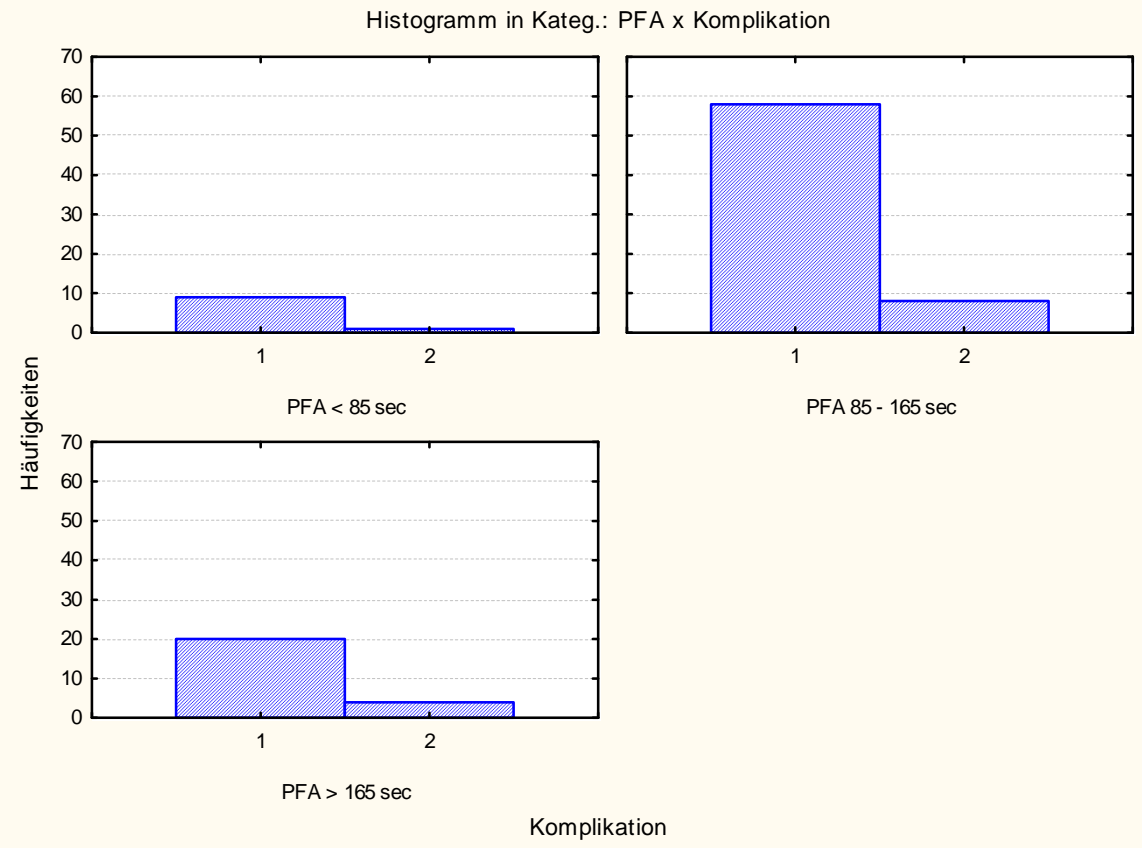

Abb. 9: Beziehung zwischen PFA-100 ${ }^{\mathrm{TM}}$ und Komplikation.

1 = keine Komplikation, 2 = Komplikation 


\subsubsection{Beziehung zwischen PFA-100 ${ }^{\mathrm{TM}}$ und Komplikationsart}

1) Makrohämaturie

Die Patienten mit einer neu aufgetretenen Makrohämaturie zeigten keinen signifikanten Unterschied in der prozentualen Verteilung auf die PFA-Gruppen zu den Patienten ohne Komplikation (p 0,2578).

2) Blutung

Es ergab sich kein signifikanter Unterschied in der prozentualen Verteilung auf die PFA-Gruppen im Vergleich der Patienten mit und ohne Blutung nach Biopsie (p 0,3173).

3) Klinisch relevanter $\mathrm{Hb}$-Abfall

Die Patienten mit einem klinisch relevanten $\mathrm{Hb}$-Abfall wiesen keinen signifikanten Unterschied in der prozentualen Verteilung auf die PFA-Gruppen auf zu den Patienten ohne Komplikation ( $p$ 0,1418).

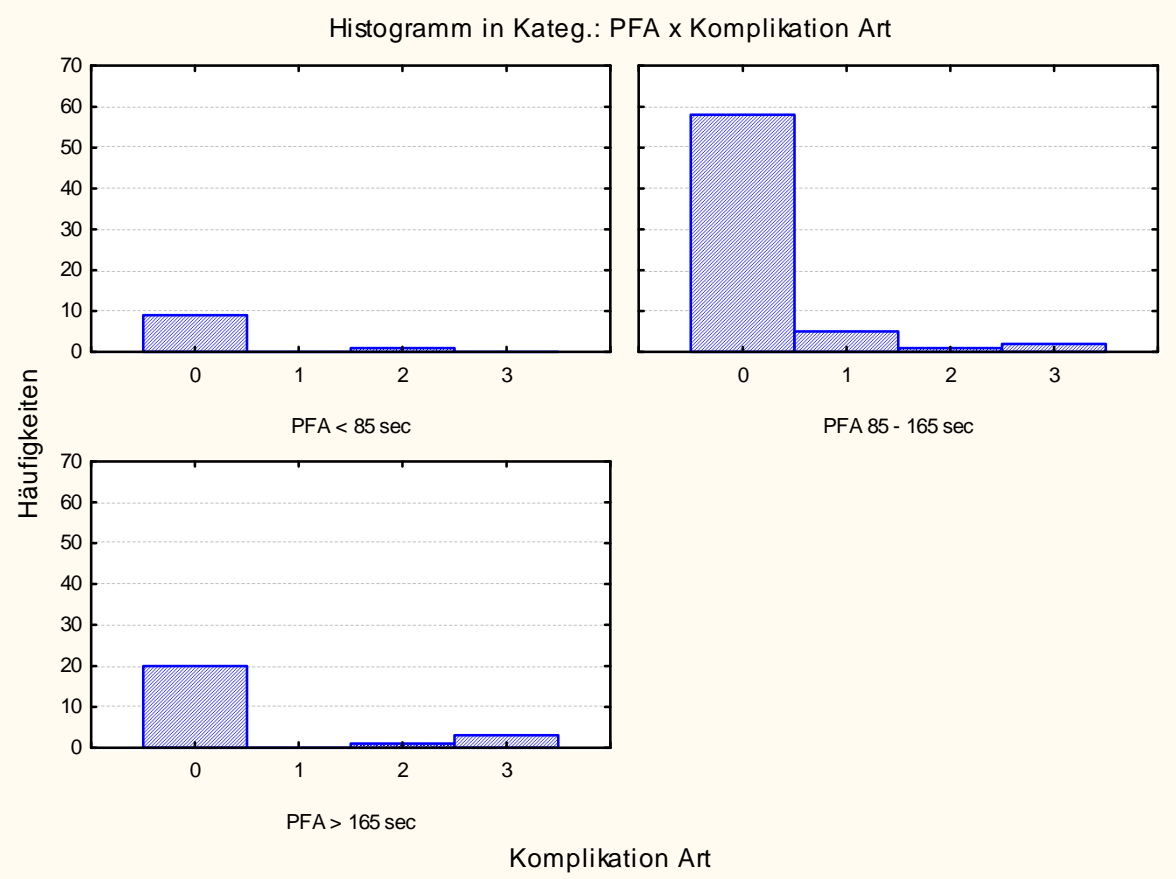

Abb. 10: Beziehung zwischen PFA-100 ${ }^{\mathrm{TM}}$ und Komplikationsart.

0 = keine Komplikation, 1 = Makrohämaturie, 2 = Blutung, 3 = Hb-Abfall

PFA-100 ${ }^{\mathrm{TM}}$ erwies sich demnach in dieser Studie nicht als nützlicher Risikofaktor, um das individuelle Risiko für Komplikationen nach perkutaner Nierenbiopsie besser prognostizieren zu können. 


\subsection{Das Geschlecht als Parameter zur Evaluation von Risiken}

Durchgeführt wurde jeweils der Chi-Quadrat-Test nach Pearson.

\subsubsection{Beziehung zwischen Geschlecht und Hämatom}

In der prozentualen Verteilung auf die beiden Geschlechter zeigte sich kein signifikanter Unterschied zwischen den Patienten mit und ohne Hämatomentwicklung ( $p$, 8001).

\subsubsection{Beziehung zwischen Geschlecht und $\mathrm{Hb}-A b f a l l>1 \mathrm{~g} / \mathrm{dl}$}

Die Patienten mit $\mathrm{Hb}$-Abfall $>1 \mathrm{~g} / \mathrm{dl}$ innerhalb der ersten 24 Stunden nach Biopsie unterschieden sich nicht signifikant in der prozentualen Verteilung auf die beiden Geschlechter von den Patienten ohne Hb-Abfall > $1 \mathrm{~g} / \mathrm{dl}$ ( $p$ 0,5344).

\subsubsection{Beziehung zwischen Geschlecht und Komplikation}

Es ergab sich kein signifikanter Unterschied in der prozentualen Verteilung auf die beiden Geschlechter im Vergleich der Patienten mit und ohne Komplikation (p 0,3791).

\subsubsection{Beziehung zwischen Geschlecht und Komplikationsart}

1) Makrohämaturie

Die Patienten mit einer neu aufgetretenen Makrohämaturie wiesen keinen signifikanten Unterschied in der prozentualen Verteilung auf die beiden Geschlechter auf zu den Patienten ohne Komplikation (p 0,4028).

2) Blutung

Die Patienten mit einer Blutung nach Biopsie unterschieden sich nicht signifikant in der prozentualen Verteilung auf die beiden Geschlechter von den Patienten ohne Komplikation ( $p$ 0,7575).

3) Klinisch relevanter $\mathrm{Hb}$-Abfall

Die Patienten mit einem klinisch relevanten $\mathrm{Hb}$-Abfall zeigten keinen signifikanten Unterschied in der prozentualen Verteilung auf die beiden Geschlechter zu den Patienten ohne Komplikation (p 0,0509). Die männlichen Patienten wiesen dabei tendenziell häufiger einen klinisch relevanten $\mathrm{Hb}$-Abfall auf als die weiblichen. 
Histogramm in Kateg.: Geschlecht x Komplikation Art

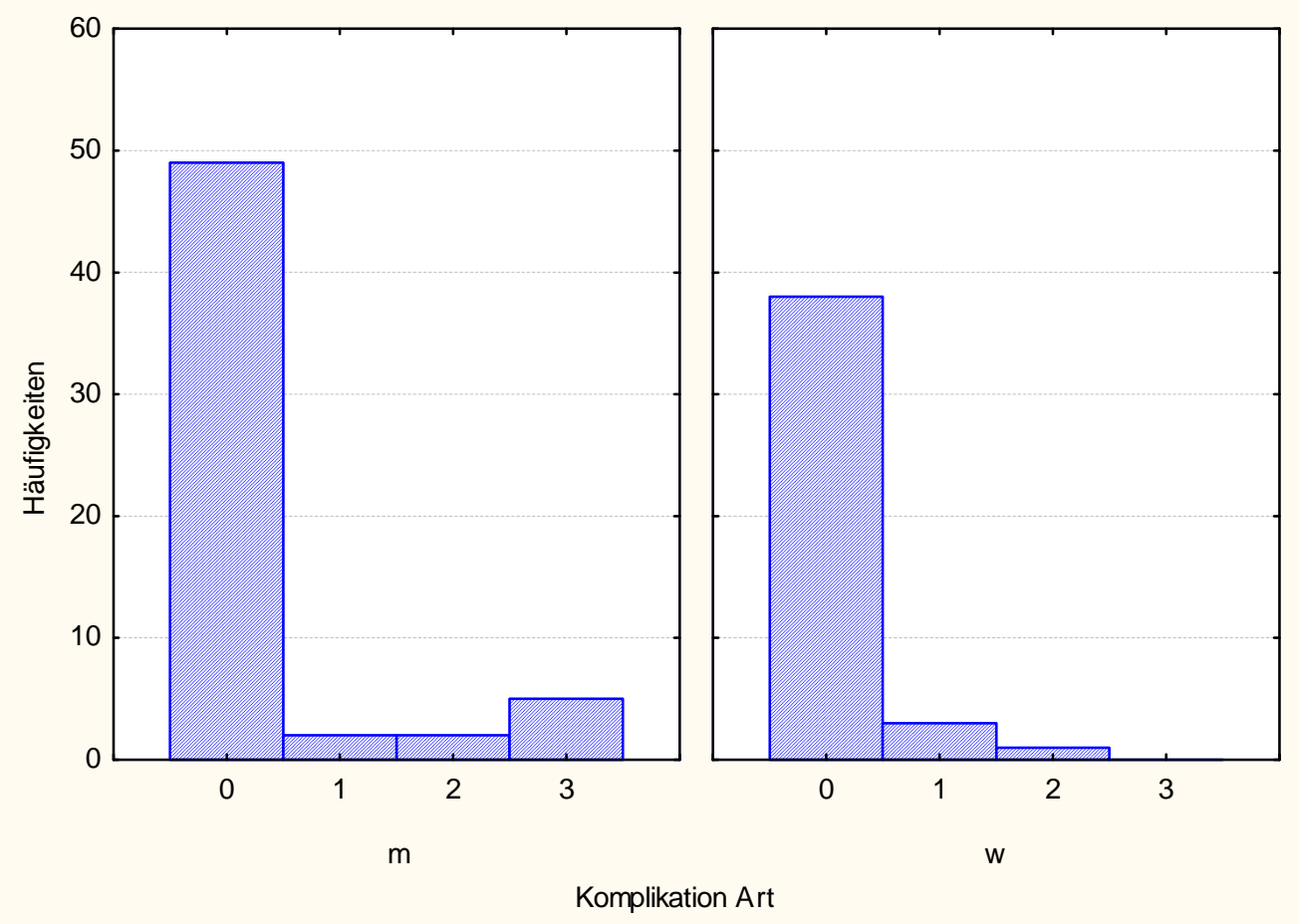

Abb. 11: Beziehung zwischen Geschlecht und Komplikationsart.

$0=$ keine Komplikation, $1=$ Makrohämaturie, 2 = Blutung, $3=\mathrm{Hb}$-Abfall

\subsection{Das Alter als Parameter zur Evaluation von Risiken}

Das Alter wurde bei allen 100 Patienten bestimmt. Es ergab sich ein Mittelwert von 49,57 Jahren bei einem Minimum von 16 Jahren und einem Maximum von 76 Jahren. Der Median lag bei 52 Lebensjahren.

Durchgeführt wurde jeweils der t-Test für unabhängige Stichproben.

\subsubsection{Beziehung zwischen Alter und Hämatom}

Es ergab sich kein signifikanter Unterschied in der Altersverteilung im Vergleich der Patienten mit und ohne Hämatom ( $p$ 0,0545), wobei jüngere Patienten tendenziell häufiger ein Hämatom im Verlauf entwickelten als die älteren. 


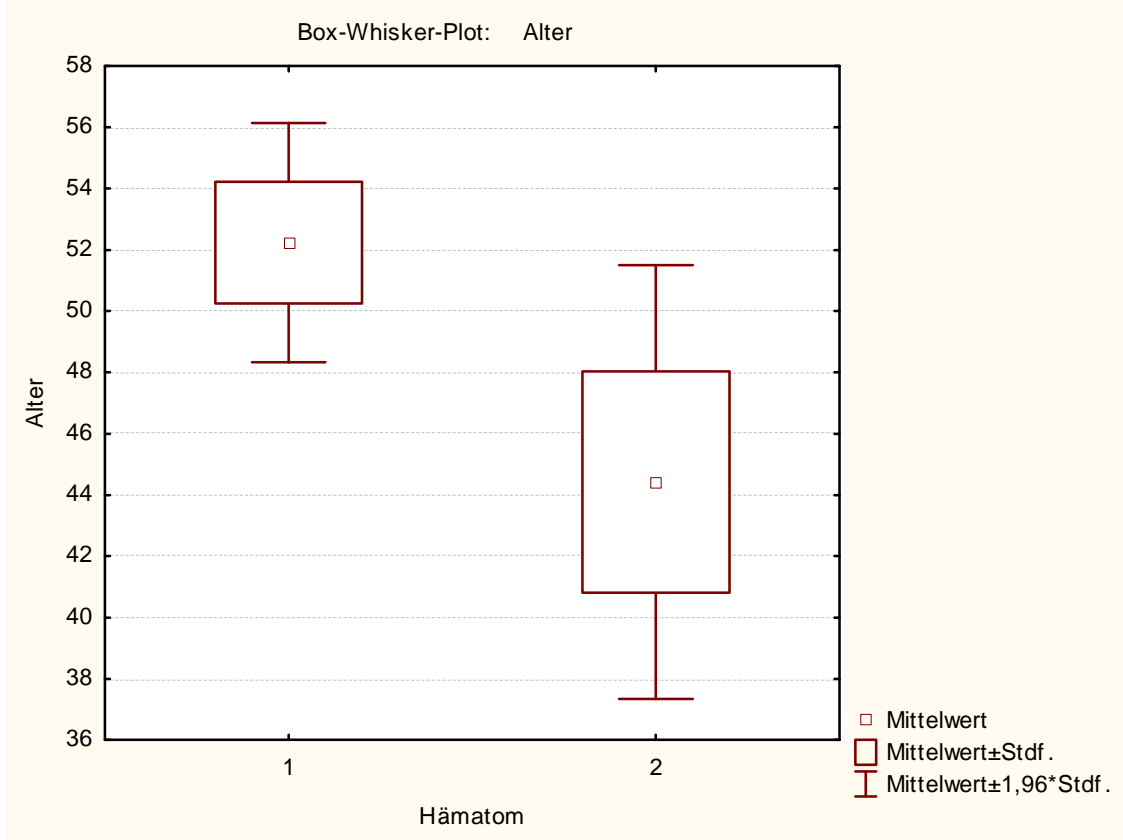

Abb. 12: Beziehung zwischen Alter und Hämatom.

1 = kein Hämatom, 2 = Hämatom

\subsubsection{Beziehung zwischen Alter und Hb-Abfall $>1 \mathrm{~g} / \mathrm{dl}$}

Die Patienten mit einem Hb-Abfall $>1 \mathrm{~g} / \mathrm{dl}$ in den ersten 24 Stunden nach Biopsie unterschieden sich in der Altersverteilung nicht signifikant von den Patienten ohne

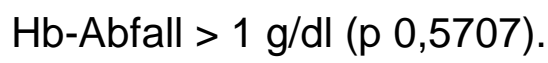

\subsubsection{Beziehung zwischen Alter und Komplikation}

Die Patienten mit einer Komplikation nach Biopsie wiesen keinen signifikanten Unterschied in der Altersverteilung auf verglichen mit den Patienten ohne Komplikation ( $p$ 0,5166).

\subsubsection{Beziehung zwischen Alter und Komplikationsart}

1) Makrohämaturie

Die Patienten mit einer neu aufgetretenen Makrohämaturie zeigten keinen signifikanten Unterschied in der Altersverteilung zu den Patienten ohne Komplikation (p 0,6958).

2) Blutung

Die Patienten mit einer Blutung nach Biopsie wiesen keinen signifikanten Unterschied in der Altersverteilung auf zu den Patienten ohne Komplikation ( $p$ 0,2178). 
3) Klinisch relevanter Hb-Abfall

Die Patienten mit einem klinisch relevanten $\mathrm{Hb}$-Abfall unterschieden sich in der Altersverteilung nicht signifikant von den Patienten ohne Komplikation ( $p$ 0,8053).

\subsection{Die Nadeldicke als Parameter zur Evaluation von Risiken}

Durchgeführt wurde jeweils der Chi-Quadrat-Test nach Pearson.

\subsubsection{Beziehung zwischen Nadeldicke und Hämatom}

Die Patienten mit Hämatomentwicklung zeigten keinen signifikanten Unterschied in der prozentualen Verteilung auf die Gruppen mit unterschiedlicher Nadeldicke zu den Patienten ohne Hämatom (p 0,8259).

\subsubsection{Beziehung zwischen Nadeldicke und Hb-Abfall $>1 \mathrm{~g} / \mathrm{dl}$}

Die Patienten mit Hb-Abfall > $1 \mathrm{~g} / \mathrm{dl}$ innerhalb der ersten 24 Stunden nach Biopsie wiesen keinen signifikanten Unterschied in der prozentualen Verteilung auf die Gruppen mit unterschiedlicher Nadeldicke auf zu den Patienten ohne Hb-Abfall > $1 \mathrm{~g} / \mathrm{dl}$ (p 0,4222).

\subsubsection{Beziehung zwischen Nadeldicke und Komplikation}

Es ergab sich kein signifikanter Unterschied in der prozentualen Verteilung auf die Gruppen mit unterschiedlicher Nadeldicke im Vergleich der Patienten mit und ohne Hämatom ( $p$ 0,3632).

\subsubsection{Beziehung zwischen Nadeldicke und Komplikationsart}

1) Makrohämaturie

Die Patienten mit einer neu aufgetretenen Makrohämaturie waren nicht signifikant unterschiedlich in der prozentualen Verteilung auf die Gruppen mit unterschiedlicher Nadeldicke von den Patienten ohne Komplikation ( $p$,7799).

2) Blutung 
Die Patienten mit einer Blutung nach Biopsie unterschieden sich nicht signifikant in der prozentualen Verteilung auf die Gruppen mit unterschiedlicher Nadeldicke von den Patienten ohne Komplikation ( $p$ 0,6087).

3) Klinisch relevanter Hb-Abfall

Die Patienten mit einem klinisch relevanten $\mathrm{Hb}$-Abfall wiesen keinen signifikanten Unterschied in der prozentualen Verteilung auf die Gruppen mit unterschiedlicher Nadeldicke auf zu den Patienten ohne Komplikation ( $p$ 0,5818).

\subsection{Die Dauer des stationären Aufenthaltes als Parameter zur Evaluation von Risiken}

Die Dauer des stationären Aufenthaltes betrug im Mittel 14,2 Tage (Median 9 Tage). Das Minimum lag bei einer Aufenthaltsdauer von 2 Tagen, das Maximum bei 91 Tagen. Patienten mit einer langen Aufenthaltsdauer befanden sich meist bereits in stationärer Behandlung, bevor eine Nierenbiopsie geplant wurde, oder entwickelten nach der Biopsie andere Probleme, die nicht in direktem Zusammenhang mit der Biopsie standen, sodass der stationäre Aufenthalt verlängert werden musste. Die Dauer des stationären Aufenthaltes ist somit auch nur bedingt zur Diskussion über einen idealen Beobachtungszeitraum nach perkutaner Nierenbiopsie geeignet.

Durchgeführt wurde jeweils der t-Test für unabhängige Stichproben.

\subsubsection{Beziehung zwischen stationärem Aufenthalt und Hämatom}

Bei den Patienten mit Hämatomentwicklung nach der Biopsie ergab sich kein signifikanter Unterschied in der Dauer des stationären Aufenthaltes zu den Patienten, die kein Hämatom entwickelten ( $p$ 0,7526).

\subsubsection{Beziehung zwischen stationärem Aufenthalt und $\mathrm{Hb}-A b f a l l ~>1 \mathrm{~g} / \mathrm{dl}$}

Die Patienten mit einem $\mathrm{Hb}$-Abfall $>1 \mathrm{~g} / \mathrm{dl}$ in den ersten 24 Stunden nach Biopsie zeigten keinen signifikanten Unterschied in der Dauer des stationären Aufenthaltes zu den Patienten ohne Hb-Abfall > $1 \mathrm{~g} / \mathrm{dl}$ ( $p$ 0,9299). 


\subsubsection{Beziehung zwischen stationärem Aufenthalt und Komplikation}

Die Patienten mit einer Komplikation nach Biopsie unterschieden sich nicht signifikant in der Dauer des stationären Aufenthaltes von den Patienten ohne Komplikation ( $p$ 0,8736).

\subsubsection{Beziehung zwischen stationärem Aufenthalt und Komplikationsart}

1) Makrohämaturie

Die Patienten mit einer neu aufgetretenen Makrohämaturie zeigten keinen signifikanten Unterschied in der Dauer des stationären Aufenthaltes zu den Patienten ohne Komplikation ( $p$ 0,9621).

2) Blutung

Es ergab sich kein signifikanter Unterschied in der Dauer des stationären Aufenthaltes im Vergleich der Patienten mit einer Blutung nach Biopsie und der Patienten ohne Komplikation ( $p$ 0,2232).

3) Klinisch relevanter $\mathrm{Hb}$-Abfall

Die Patienten mit einem klinisch relevanten $\mathrm{Hb}$-Abfall wiesen keinen signifikanten Unterschied in der Dauer des stationären Aufenthaltes auf zu den Patienten ohne Komplikation ( $p$ 0,2447).

\subsection{Der Hämoglobingehalt $(\mathrm{Hb})$ als Parameter zur Evaluation von Risiken}

Der Hämoglobingehalt konnte retrospektiv bei 97 Patienten erfasst werden. Im Mittel betrug der Hb-Wert 12,1 g/dl mit dem Median bei 11,9 g/dl. Der Minimalwert lag bei $8,8 \mathrm{~g} / \mathrm{dl}$, das Maximum bei 17,0 $\mathrm{g} / \mathrm{dl}$.

Durchgeführt wurde jeweils der t-Test für unabhängige Stichproben.

\subsubsection{Beziehung zwischen $\mathrm{Hb}$ und Hämatom}

Bei Betrachtung der Hämatomentwicklung nach Biopsie in Bezug auf den $\mathrm{Hb}$ Wert, ergab sich signifikanter Unterschied in der Höhe des Hb-Spiegels im Vergleich der Patienten mit und ohne Hämatomentwicklung ( $p 0,7895)$. 


\subsubsection{Beziehung zwischen $\mathrm{Hb}$ und $\mathrm{Hb}$-Abfall $>1 \mathrm{~g} / \mathrm{dl}$}

Die Patienten mit einem Hb-Abfall $>1 \mathrm{~g} / \mathrm{dl}$ in den ersten 24 Stunden nach Biopsie zeigten keinen signifikanten Unterschied in der Höhe des Hb-Wertes zu den

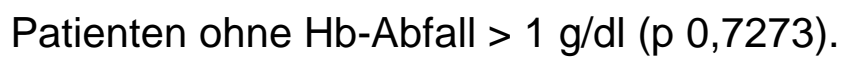

\subsubsection{Beziehung zwischen $\mathrm{Hb}$ und Komplikation}

Es ergab sich ein signifikanter Unterschied in der Höhe des Hb-Wertes zwischen den Patienten mit Komplikation und den Patienten ohne Komplikation ( $p$ 0,0195). Patienten mit einem niedrigeren $\mathrm{Hb}$-Wert wiesen häufiger Komplikationen auf.

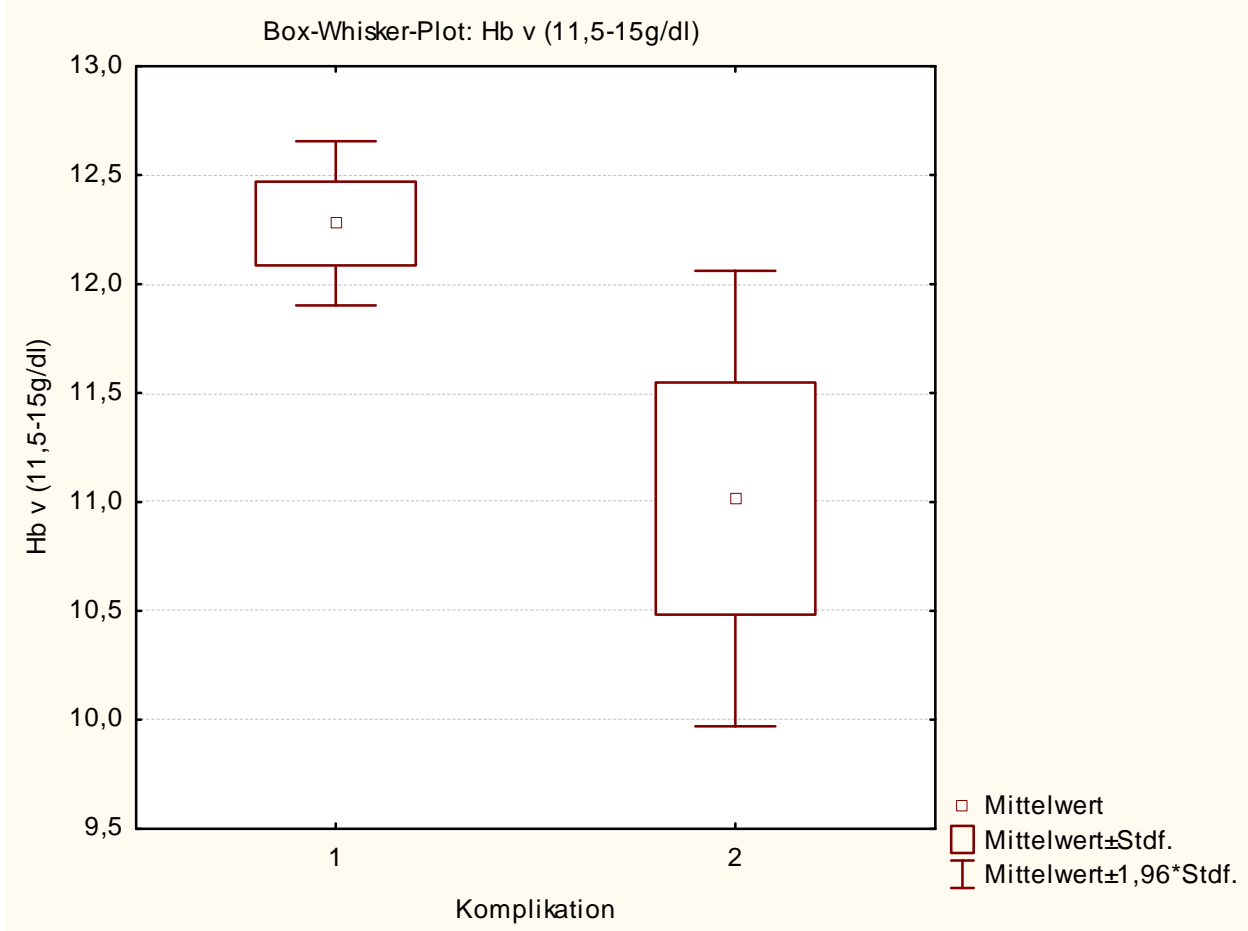

Abb. 13: Beziehung zwischen $\mathrm{Hb}$ und Komplikation.

1 = keine Komplikation, 2 = Komplikation

\subsubsection{Beziehung zwischen $\mathrm{Hb}$ und Komplikationsart}

1) Makrohämaturie

Die Patienten mit einer neu aufgetretenen Makrohämaturie unterschieden sich nicht signifikant in der Höhe des Hb-Wertes von den Patienten ohne Komplikation (p 0,8838). 
2) Blutung

Es ergab sich kein signifikanter Unterschied in der Höhe des Hb-Wertes im Vergleich der Patienten mit einer Blutung nach Biopsie und der Patienten ohne Komplikation ( $p$ 0,0999). Diese Tendenz deutet zwar darauf hin, dass Patienten mit einem niedrigen $\mathrm{Hb}$-Wert vor Biopsie eher zu Blutungen neigen als Patienten mit einem hohen Hb-Wert. Aufgrund fehlender Signifikanz ist dieses Ergebnis aber nicht zur Vorhersage eines individuellen Risikos geeignet.

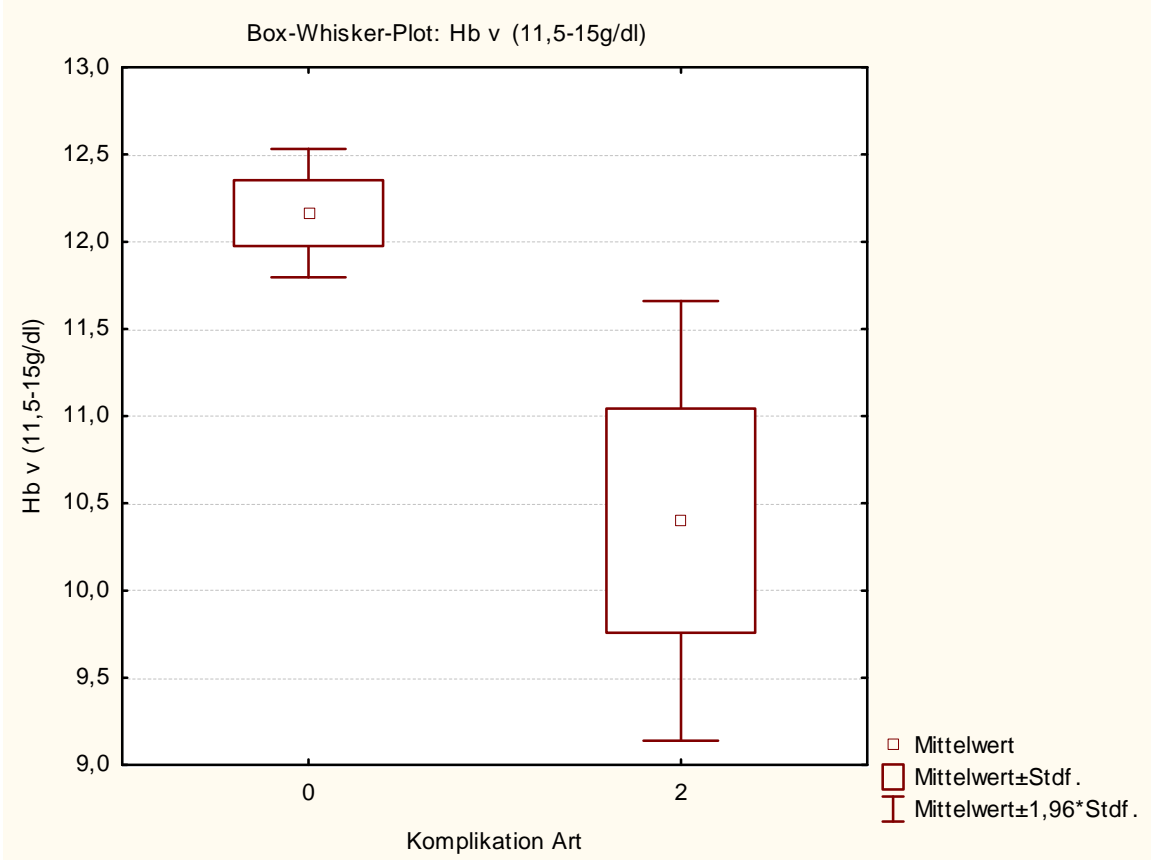

Abb. 14: Beziehung zwischen $\mathrm{Hb}$ und Komplikationsart.

$0=$ keine Komplikation, 2 = Blutung

3) Klinisch relevanter $\mathrm{Hb}$-Abfall

Bei der Betrachtung des $\mathrm{Hb}$-Wertes bei Patienten mit klinisch relevantem $\mathrm{Hb}$ Abfall und Patienten ohne Komplikation stellte sich heraus, dass Patienten mit einem niedrigen $\mathrm{Hb}$-Wert signifikant häufiger einen klinisch relevanten $\mathrm{Hb}$-Abfall hatten als Patienten mit einem höheren $\mathrm{Hb}$-Wert. Diese blieben häufiger ohne Komplikation (p 0,0053). 


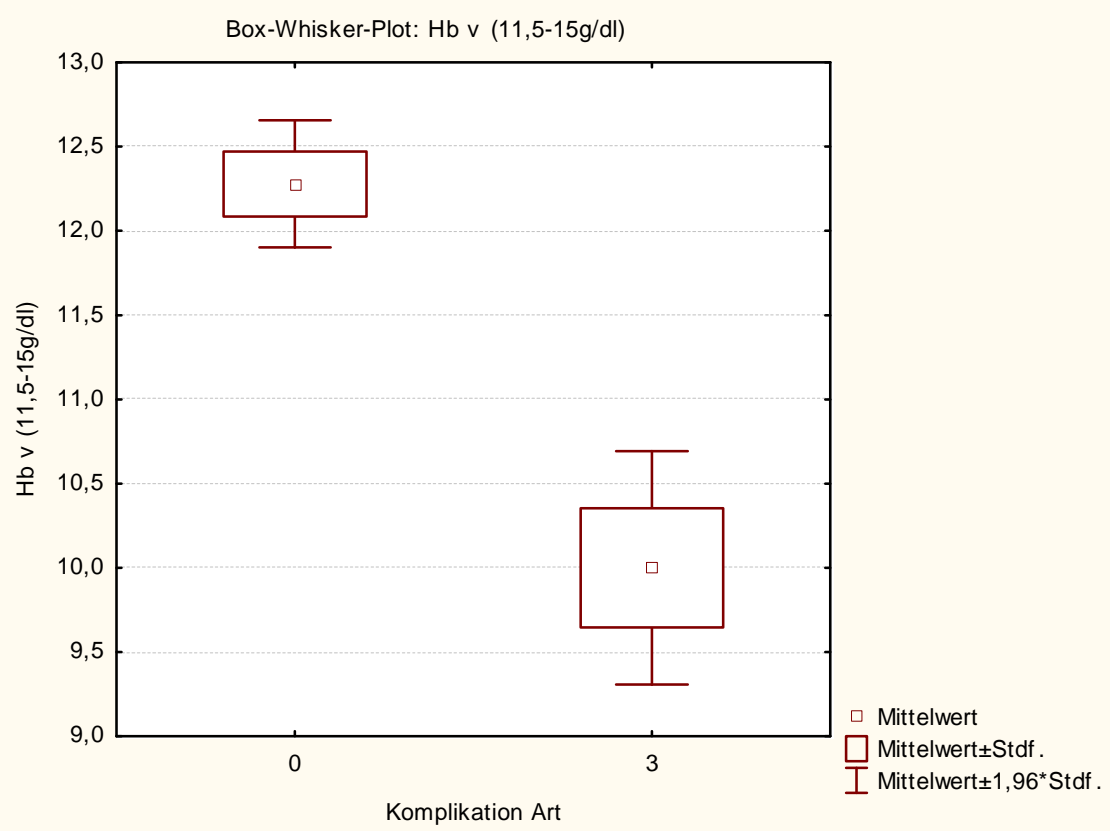

Abb. 15: Beziehung zwischen $\mathrm{Hb}$ und Komplikationsart $\rightarrow \mathrm{Hb}$-Abfall.

$0=$ keine Komplikation, $3=\mathrm{Hb}$-Abfall

\subsection{Der Quickwert als Parameter zur Evaluation von Risiken}

\subsubsection{Berechnung mit ordinalskalierten Werten}

Der Quickwert konnte bei 96 von 100 Patienten retrospektiv nachvollzogen werden. Es ergab sich ein Mittelwert von 110,4 \% (Median 112,5\%) mit einem Minimum bei 39\% und einem Maximum bei 130\%. Werte über $130 \%$ konnten nicht genau angegeben werden und wurden deshalb mit 130 gleichgesetzt. Durchgeführt wurde jeweils der t-Test für unabhängige Stichproben.

\subsubsection{Beziehung zwischen Quick und Hämatom}

Die Patienten mit Hämatomentwicklung nach der Biopsie zeigten keinen signifikanten Unterschied in der Höhe des Quickwertes zu den Patienten, die kein Hämatom entwickelten ( $p$ 0,3198).

\subsubsection{Beziehung zwischen Quick und Hb-Abfall $>1 \mathrm{~g} / \mathrm{dl}$}

Die Patienten mit einem Hb-Abfall $>1 \mathrm{~g} / \mathrm{dl}$ in den ersten 24 Stunden nach Biopsie wiesen keinen signifikanten Unterschied in der Höhe des Quickwertes auf zu den Patienten ohne Hb-Abfall > $1 \mathrm{~g} / \mathrm{dl}(\mathrm{p} \mathrm{0,1289)}$. 


\subsubsection{Beziehung zwischen Quick und Komplikation}

Die Patienten mit einer Komplikation nach Biopsie zeigten keinen signifikanten Unterschied in der Höhe des Quickwertes zu den Patienten ohne Komplikation ( $p$ 0,3092).

\subsubsection{Beziehung zwischen Quick und Komplikationsart}

1) Makrohämaturie

Die Patienten mit einer neu aufgetretenen Makrohämaturie unterschieden sich nicht signifikant in der Höhe des Quickwertes von den Patienten ohne Komplikation ( $p$ 0,3464).

2) Blutung

Da die Patienten mit einer Blutung nach Biopsie sich in ihrem Quickwert nicht signifikant von den Patienten ohne Komplikation unterschieden, konnte der Quickwert nicht als prognostischer Faktor für Blutungskomplikationen nach Nierenbiopsien bestätigt werden ( $p 0,3785$ ).

3) Klinisch relevanter Hb-Abfall

Es ergab sich kein signifikanter Unterschied in der Höhe des Quickwertes im Vergleich der Patienten mit klinisch relevantem $\mathrm{Hb}$-Abfall und der Patienten ohne Komplikation ( $p$,2241).

\subsubsection{Berechnung mit nominalskalierten Werten}

Aus den oben angeführten Gründen erfolgte nun eine zweite Berechnung. Dazu wurden die Patienten in verschiedene Gruppen unterteilt: 1: Quick $<70 \%, 2$ : Quick 70 - 120\%, 3: Quick > 120\%.

Durchgeführt wurde jeweils der Chi-Quadrat-Test nach Pearson.

\subsubsection{Beziehung zwischen Quick und Hämatom}

Die Patienten mit Hämatomentwicklung zeigten keinen signifikanten Unterschied in der prozentualen Verteilung auf die Quickwertgruppen zu den Patienten ohne Hämatom ( $p$ 0,0639). Diese Tendenz konnte allerdings mit den ordinalskalierten Daten nicht bestätigt werden und bleibt daher ohne Einfluss. 


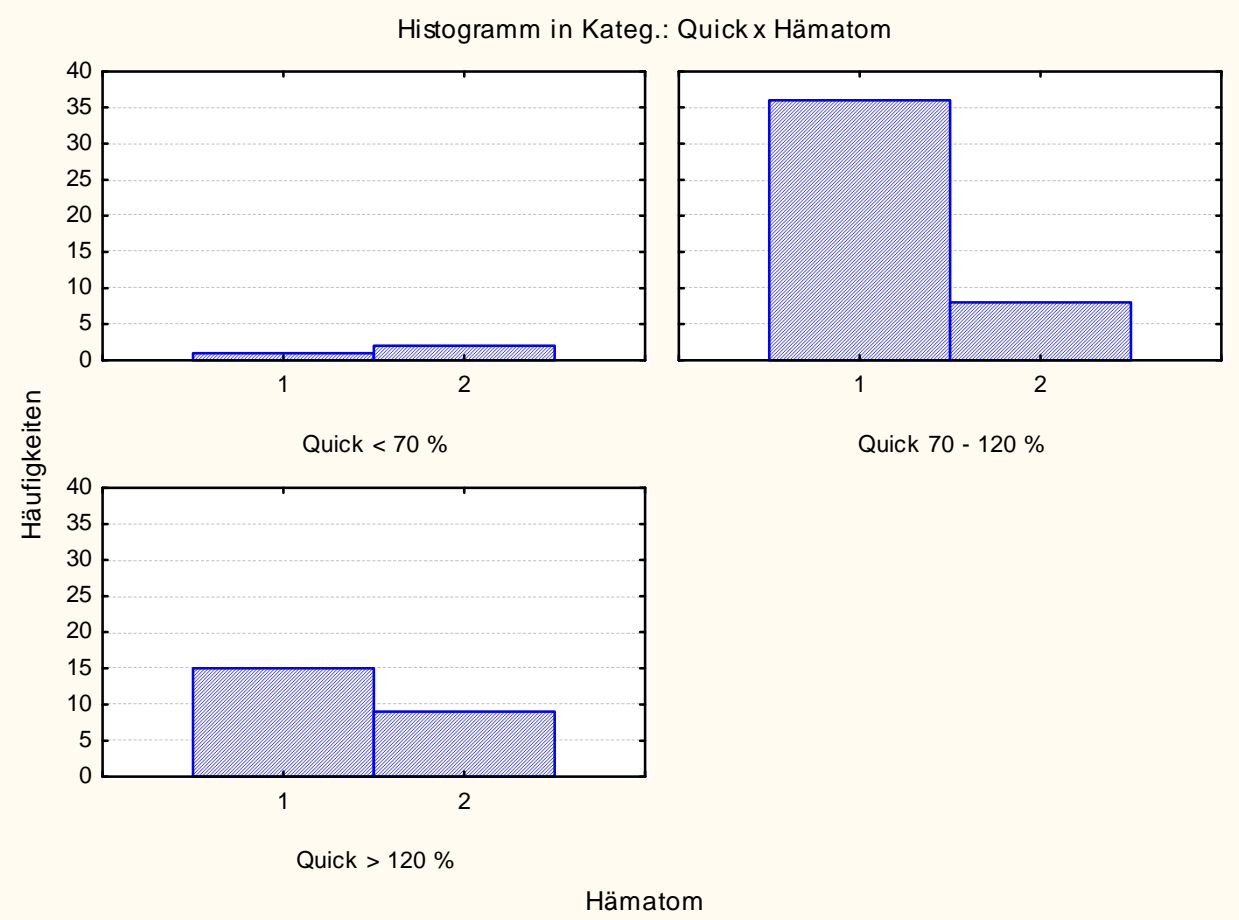

Abb. 16: Beziehung zwischen Quick und Hämatom. 1 = kein Hämatom, 2 = Hämatom

\subsubsection{Beziehung zwischen Quick und Hb-Abfall $>1 \mathrm{~g} / \mathrm{dl}$}

Die Patienten mit $\mathrm{Hb}$-Abfall > $1 \mathrm{~g} / \mathrm{dl}$ innerhalb der ersten 24 Stunden nach Biopsie unterschieden sich nicht signifikant in der prozentualen Verteilung auf die Quickwertgruppen von den Patienten ohne Hb-Abfall > $1 \mathrm{~g} / \mathrm{dl}$ ( $p$ 0,5504).

\subsubsection{Beziehung zwischen Quick und Komplikation}

Die Patienten mit einer Komplikation nach Biopsie wiesen keinen signifikanten Unterschied in der prozentualen Verteilung auf die Quickwertgruppen auf zu den Patienten ohne Komplikation ( $p$ 0,6795).

\subsubsection{Beziehung zwischen Quick und Komplikationsart}

1) Makrohämaturie

Auch für die Makrohämaturie ergab sich kein signifikantes Ergebnis in Bezug auf die Höhe des Quickwertes bei dem Vergleich der Patienten mit Makrohämaturie und der Patienten ohne Komplikation ( $p$ 0,8845).

2) Blutung

Die Patienten mit einer Blutung nach Biopsie unterschieden sich nicht signifikant in der prozentualen Verteilung auf Quickwertgruppen von den Patienten ohne Komplikation ( $p$ 0,5566). 
3) Klinisch relevanter $\mathrm{Hb}$-Abfall

Die Patienten mit einem klinisch relevanten $\mathrm{Hb}$-Abfall wiesen keinen signifikanten Unterschied in der prozentualen Verteilung auf die Quickwertgruppen auf zu den Patienten ohne Komplikation ( $p$ 0,4168).

\subsection{Die PTT als Parameter zur Evaluation von Risiken}

Die PTT konnte bei 98 der 100 Patienten retrospektiv nachvollzogen werden. Der Mittelwert lag bei 28,0 sec (Median 27sec) mit einem Minimum bei 21sec und einem Maximum bei $54 \mathrm{sec}$.

Durchgeführt wurde jeweils der t-Test für unabhängige Stichproben.

\subsubsection{Beziehung zwischen PTT und Hämatom}

Es ergab sich kein signifikanter Unterschied in der Höhe des PTT-Wertes im Vergleich der Patienten mit und ohne Hämatom ( $p 0$,2224).

\subsubsection{Beziehung zwischen PTT und Hb-Abfall $>1 \mathrm{~g} / \mathrm{dl}$}

Die Patienten mit einem Hb-Abfall $>1 \mathrm{~g} / \mathrm{dl}$ in den ersten 24 Stunden nach Biopsie zeigten keinen signifikanten Unterschied in der Höhe des PTT-Wertes zu den

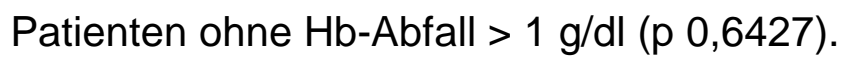

\subsubsection{Beziehung zwischen PTT und Komplikation}

Die Patienten mit einer Komplikation nach Biopsie wiesen keinen signifikanten Unterschied in der Höhe des PTT-Wertes auf zu den Patienten ohne Komplikation (p 0,1305).

\subsubsection{Beziehung zwischen PTT und Komplikationsart}

1) Makrohämaturie

Die Patienten mit einer neu aufgetretenen Makrohämaturie unterschieden sich nicht signifikant in der Höhe des PTT-Wertes von den Patienten ohne Komplikation ( $p$ 0,0620). Es zeigte sich allerdings eine Tendenz zu häufiger auftretender Makrohämaturie bei Patienten mit einer niedrigeren PTT. 


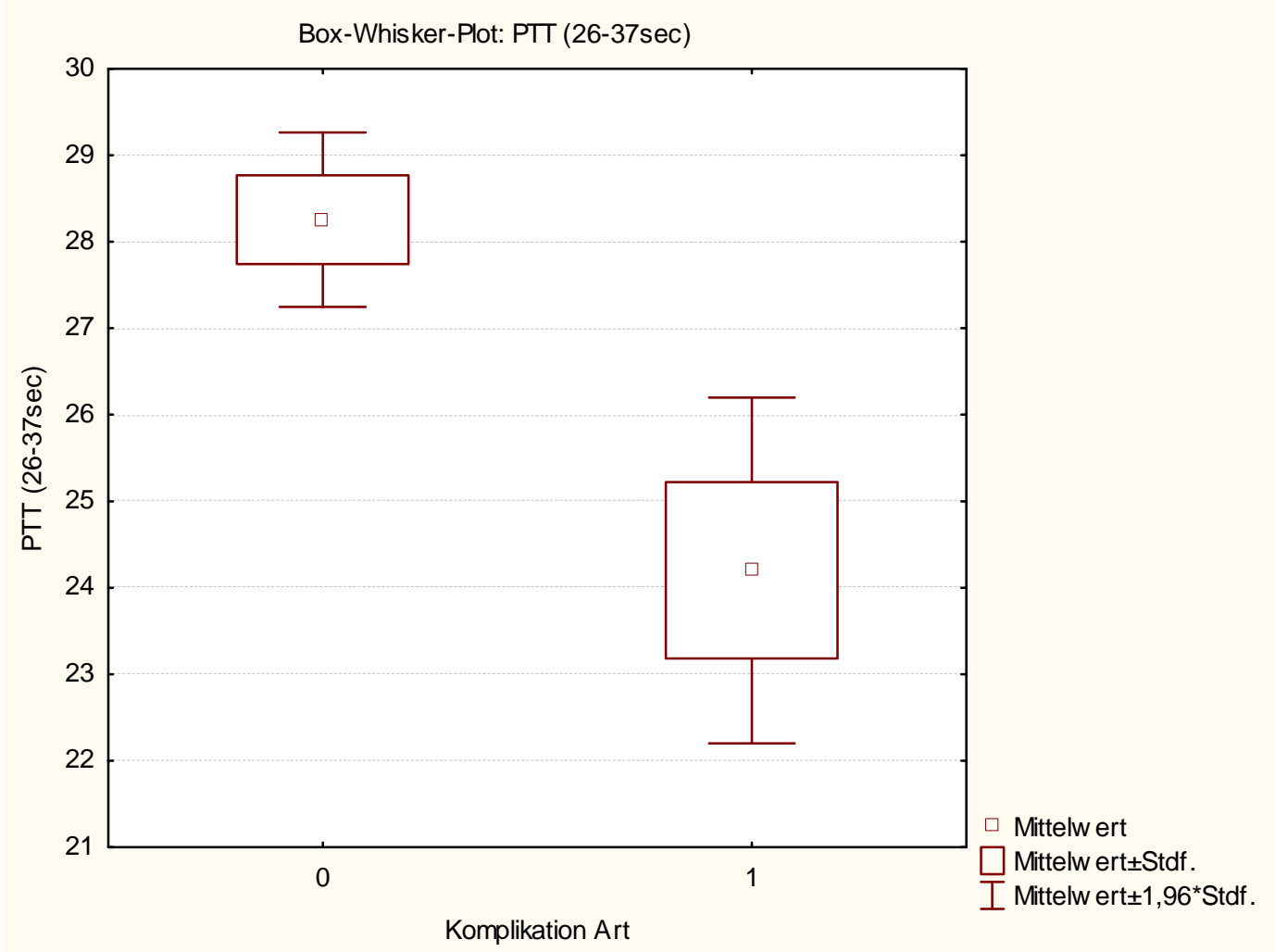

Abb. 17: Beziehung zwischen PTT und Komplikationsart $\rightarrow$ Makrohämaturie.

$0=$ keine Komplikation, $1=$ Makrohämaturie

2) Blutung

Die Patienten mit einer Blutung nach Biopsie wiesen keinen signifikanten Unterschied in der Höhe des PTT-Wertes auf zu den Patienten ohne Komplikation (p 0,6997).

3) Klinisch relevanter $\mathrm{Hb}$-Abfall

Der PTT-Wert stellte sich nicht als geeigneter Parameter zur Detektion des klinisch relevanten $\mathrm{Hb}$-Abfalls heraus, das Ergebnis des Vergleichs der Patienten mit klinisch relevantem $\mathrm{Hb}$-Abfall und ohne Komplikation blieb ohne Signifikanz (p 0,3988).

\subsection{Kreatinin als Parameter zur Evaluation von Risiken}

Bei allen 100 Patienten wurde vor der Biopsie der Kreatininspiegel bestimmt. Es ergab sich ein Mittelwert von 2,4 mg/dl (Median 1,9) mit einem Minimum bei 0,6 $\mathrm{mg} / \mathrm{dl}$ und einem Maximum bei 9,5 mg/dl.

Durchgeführt wurde jeweils der t-Test für unabhängige Stichproben. 


\subsubsection{Beziehung zwischen Kreatinin und Hämatom}

Die Patienten mit Hämatomentwicklung nach der Biopsie zeigten keinen signifikanten Unterschied in der Höhe des Kreatininwertes zu den Patienten, die kein Hämatom entwickelten ( $p$ 0,1631).

\subsubsection{Beziehung zwischen Kreatinin und $\mathrm{Hb}-A b f a l l ~>1 \mathrm{~g} / \mathrm{dl}$}

Die Patienten mit einem Hb-Abfall $>1 \mathrm{~g} / \mathrm{dl}$ in den ersten 24 Stunden nach Biopsie wiesen keinen signifikanten Unterschied in der Höhe des Kreatininwertes auf zu den Patienten ohne Hb-Abfall > $1 \mathrm{~g} / \mathrm{dl}(\mathrm{p} 0,5075)$.

\subsubsection{Beziehung zwischen Kreatinin und Komplikation}

Die Patienten mit einer Komplikation nach Biopsie hatten einen signifikant höheren Kreatininwert als die Patienten ohne Komplikation $(p<0,0001)$. Dieses Ergebnis deckt sich mit den Ergebnissen aus anderen Studien und gibt Hinweis darauf, dass Patienten mit einem hohen Kreatininspiegel gefährdeter sind, eine Komplikation zu erleiden, als Patienten mit einem normalen oder niedrigeren Kreatininspiegel vor Biopsie.

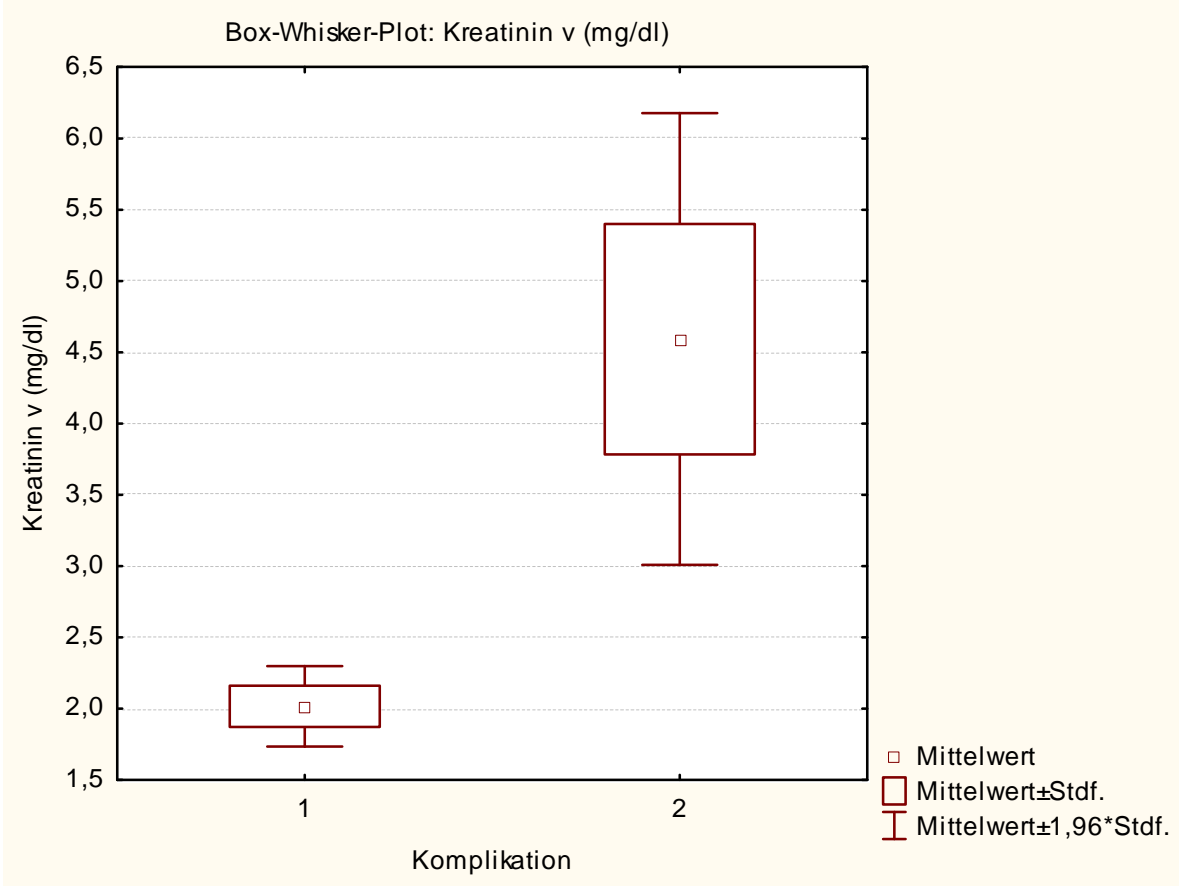

Abb. 18: Beziehung zwischen Kreatinin und Komplikation.

1 = keine Komplikation, 2 = Komplikation 


\subsubsection{Beziehung zwischen Kreatinin und Komplikationsart}

1) Makrohämaturie

Die Patienten mit einer neu aufgetretenen Makrohämaturie unterschieden sich signifikant in der Höhe des Kreatininwertes von den Patienten ohne Komplikation (p 0,0021).

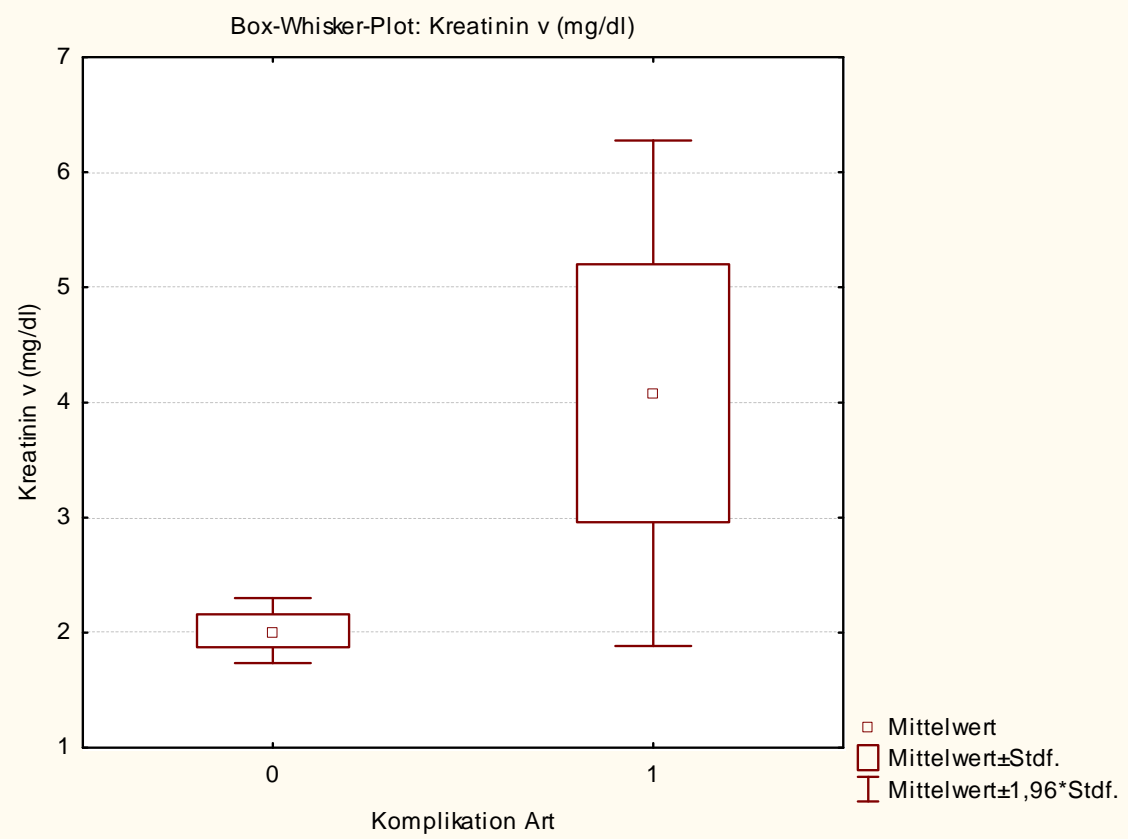

Abb. 19: Beziehung zwischen Kreatinin und Komplikationsart $\rightarrow$ Makrohämaturie.

$0=$ keine Komplikation, 1 = Makrohämaturie

2) Blutung

Auch Patienten mit einer Blutung nach Biopsie hatten einen signifikant höheren Kreatininwert als Patienten ohne Komplikation ( $p$, 0001).

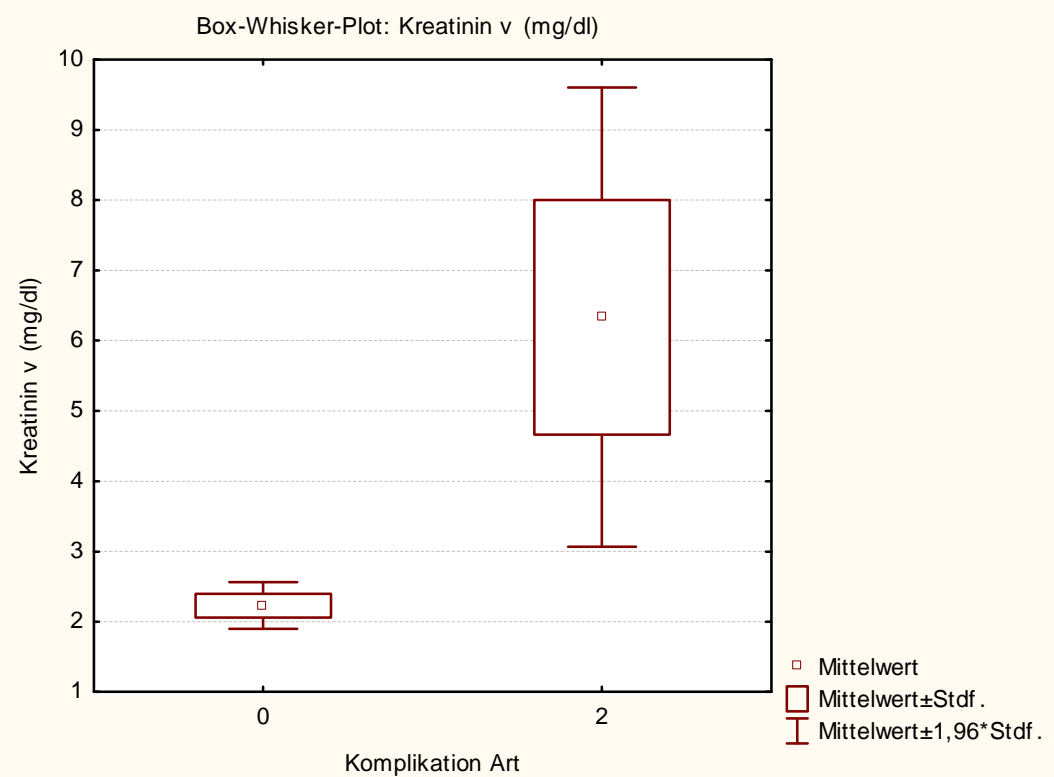

Abb. 20: Beziehung zwischen Kreatinin und Komplikationsart $\rightarrow$ Blutung. 
3) Klinisch relevanter Hb-Abfall

Es ergab sich ein signifikanter Unterschied im Vergleich des Kreatininwertes der Patienten mit klinisch relevantem Hb-Abfall zu den Patienten ohne Komplikation (p 0,0040).

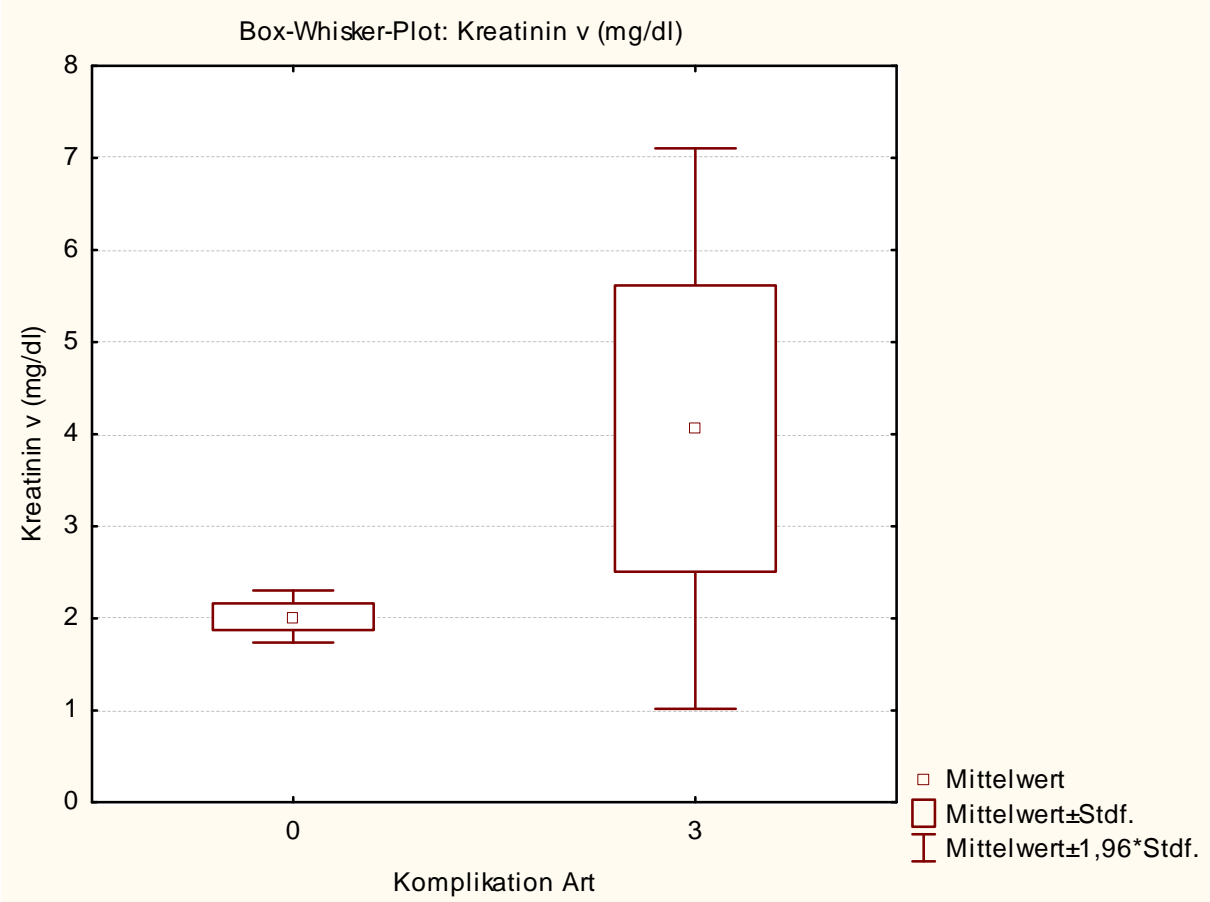

Abb. 21: Beziehung zwischen Kreatinin und Komplikationsart $\rightarrow \mathrm{Hb}$-Abfall.

$0=$ keine Komplikation, $3=\mathrm{Hb}$-Abfall

Patienten mit einem hohen Kreatininspiegel wiesen also signifikant häufiger eine der drei Komplikationsarten Makrohämaturie, Blutung oder klinisch relevanter $\mathrm{Hb}$ Abfall auf als Patienten mit einem niedrigeren Kreatininwert vor Biopsie. Daher ist zu überlegen, ob diese Patienten nach Biopsie engmaschiger kontrolliert werden sollten, um eventuelle Komplikationen möglichst frühzeitig festzustellen.

\subsection{Die Kreatininclearance als Parameter zur Evaluation von Risiken}

Die Kreatininclearance wurde bei 61 der 100 Patienten erhoben. Der Mittelwert lag bei 58,1 $\mathrm{ml} / \mathrm{min}$ (Median 49,3 $\mathrm{ml} / \mathrm{min}$ ) mit einem Minimum bei 3,0 $\mathrm{ml} / \mathrm{min}$ und einem Maximum bei 166,4 $\mathrm{ml} / \mathrm{min}$.

Durchgeführt wurde jeweils der t-Test für unabhängige Stichproben. 


\subsubsection{Beziehung zwischen Kreatininclearance und Hämatom}

Die Patienten mit Hämatomentwicklung nach der Biopsie zeigten keinen signifikanten Unterschied in der Höhe der Kreatininclearance zu den Patienten, die kein Hämatom entwickelten ( $p$ 0,7810).

\subsubsection{Beziehung zwischen Kreatininclearance und $\mathrm{Hb}-A b f a l l ~>1 \mathrm{~g} / \mathrm{dl}$}

Die Patienten mit einem Hb-Abfall $>1 \mathrm{~g} / \mathrm{dl}$ in den ersten 24 Stunden nach Biopsie wiesen keinen signifikanten Unterschied in der Kreatininclearance auf zu den Patienten ohne Hb-Abfall > $1 \mathrm{~g} / \mathrm{dl}$ ( $\mathrm{p} \mathrm{0,9313).}$

\subsubsection{Beziehung zwischen Kreatininclearance und Komplikation}

Die Patienten mit einer Komplikation nach Biopsie unterschieden sich nicht signifikant in der Kreatininclearance von den Patienten ohne Komplikation ( $p$ 0,0986). Allerdings neigten Patienten mit einer geringen Kreatininclearance eher zu Komplikationen als Patienten mit einer höheren oder normalen Clearance.

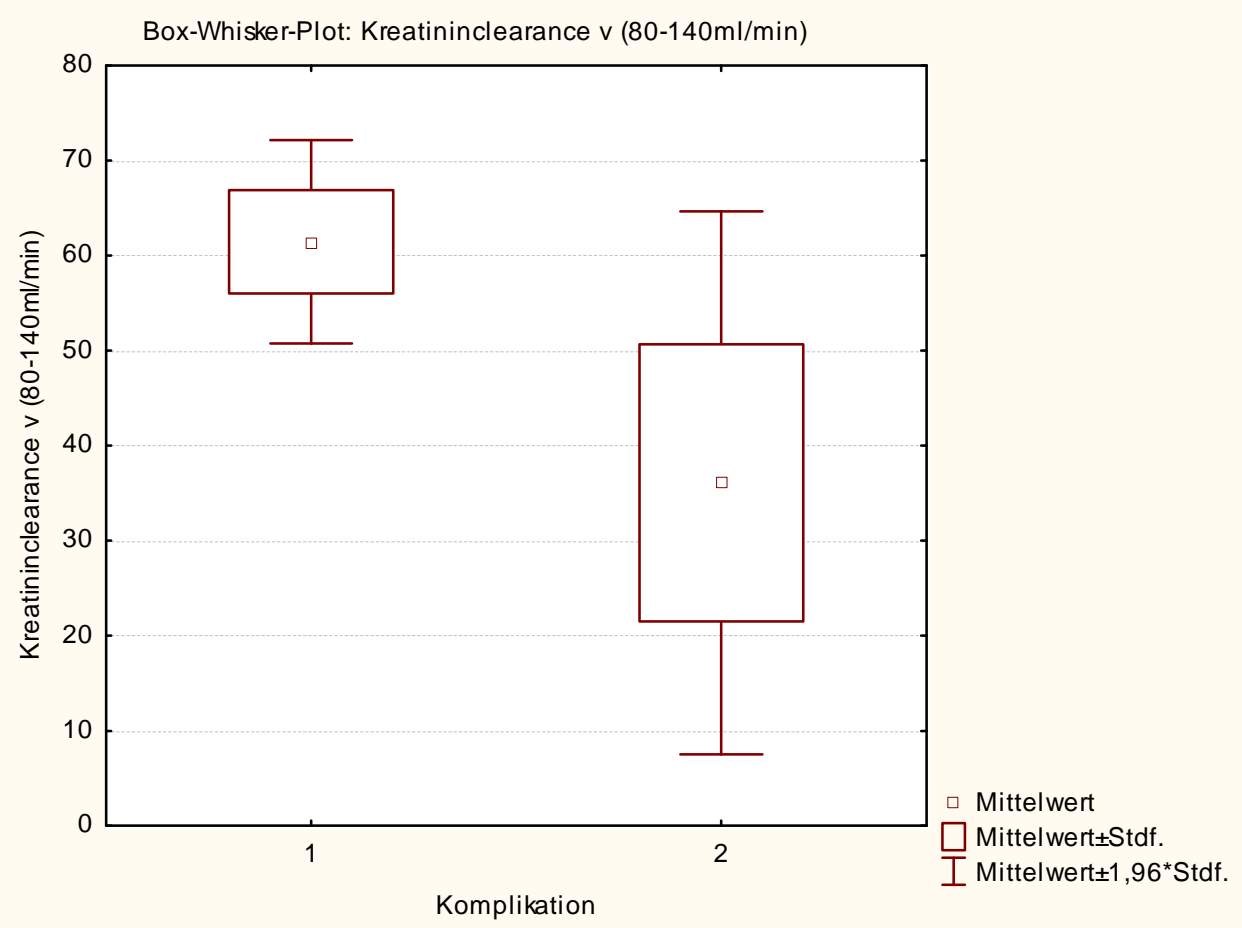

Abb. 22: Beziehung zwischen Kreatininclearance und Komplikation.

1 = keine Komplikation, 2 = Komplikation 


\subsubsection{Beziehung zwischen Kreatininclearance und Komplikationsart}

1) Makrohämaturie

Es ergab sich kein signifikanter Unterschied in der Kreatininclearance im Vergleich der Patienten mit Makrohämaturie und der Patienten ohne Komplikation (p 0,3771).

2) Blutung

Die Patienten mit einer Blutung nach Biopsie zeigten keinen signifikanten Unterschied in der Höhe der Kreatininclearance zu den Patienten ohne Komplikation ( $p$ 0,0823).

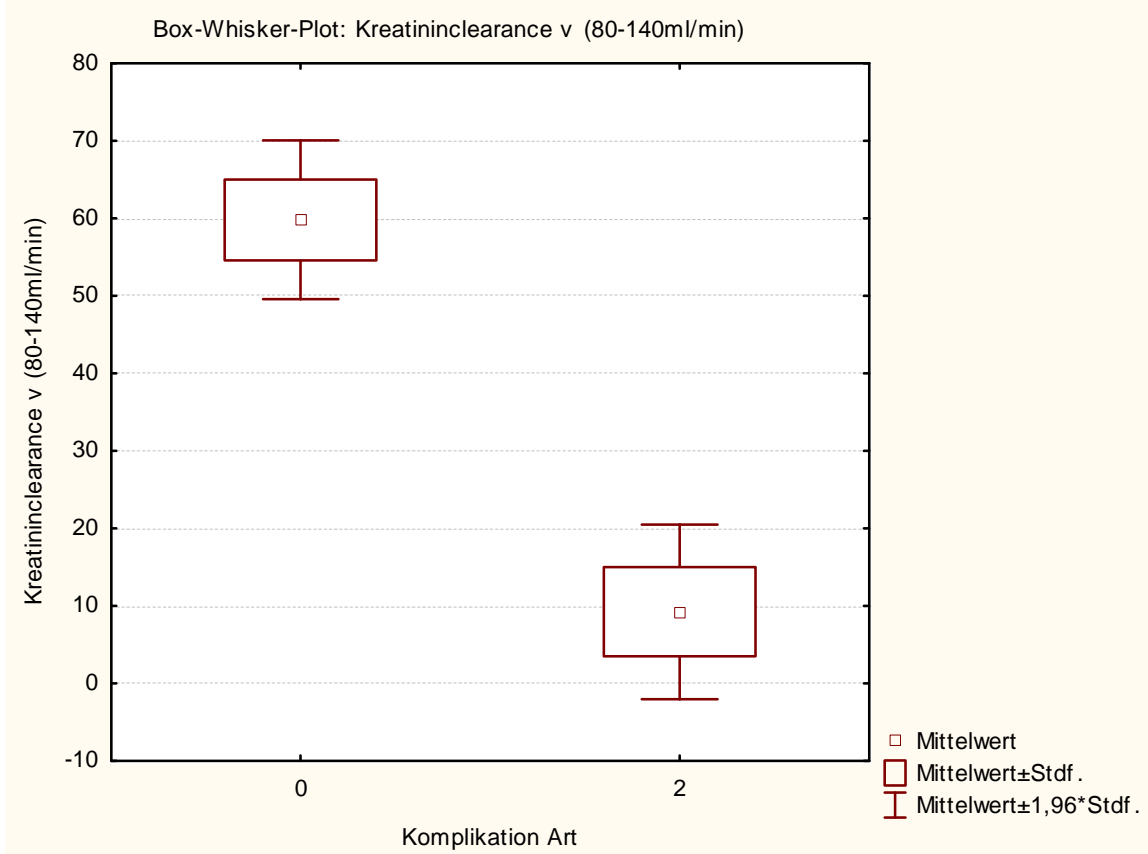

Abb. 23: Beziehung zwischen Kreatininclearance und Komplikationsart $\rightarrow$ Blutung. $0=$ keine Komplikation, 2 = Blutung

3) Klinisch relevanter $\mathrm{Hb}$-Abfall

Die Patienten mit einem klinisch relevanten $\mathrm{Hb}$-Abfall wiesen keinen signifikanten Unterschied in der Kreatininclearance auf zu den Patienten ohne Komplikation (p 0,5718). 


\subsection{Die MDRD-Formel als Parameter zur Evaluation von Risiken}

\subsubsection{Berechnung mit ordinalskalierten Werten}

Die Ergebnisse aus der MDRD-Formel konnten retrospektiv bei 86 der 100 Patienten nachvollzogen werden. Dabei lag der Mittelwert der MDRD bei 39,5 $\mathrm{ml} / \mathrm{min} / 1,73 \mathrm{~m}^{2} \mathrm{KO}$ (Median 36,3 ml/min/1,73 $\mathrm{m}^{2} \mathrm{KO}$ ) mit einem Minimum von $20,0 \mathrm{ml} / \mathrm{min} / 1,73 \mathrm{~m}^{2} \mathrm{KO}$ und einem Maximum von 60,0 ml/min/1,73 $\mathrm{m}^{2} \mathrm{KO}$. Bei der MDRD-Formel können Werte $<20$ und $>60 \mathrm{ml} / \mathrm{min} / 1,73 \mathrm{~m}^{2} \mathrm{KO}$ nicht exakt angegeben werden, deshalb wurden diese gleich $20 \mathrm{bzw} .60 \mathrm{ml} / \mathrm{min} / 1,73 \mathrm{~m}^{2} \mathrm{KO}$ gesetzt.

Durchgeführt wurde jeweils der t-Test für unabhängige Stichproben.

\subsubsection{Beziehung zwischen MDRD und Hämatom}

Die Patienten mit Hämatomentwicklung nach der Biopsie unterschieden sich nicht signifikant in der Höhe des MDRD-Wertes von den Patienten, die kein Hämatom entwickelten ( $p$ 0,8743).

\subsubsection{Beziehung zwischen MDRD und $\mathrm{Hb}-A b f a l l ~>1 \mathrm{~g} / \mathrm{dl}$}

Die Patienten mit einem Hb-Abfall > $1 \mathrm{~g} / \mathrm{dl}$ in den ersten 24 Stunden nach Biopsie zeigten keinen signifikanten Unterschied in der Höhe des MDRD-Wertes zu den

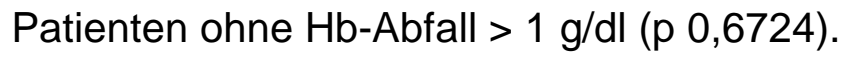

\subsubsection{Beziehung zwischen MDRD und Komplikation}

Patienten mit einer Komplikation nach Biopsie hatten einen signifikant niedrigeren MDRD-Wert als die Patienten ohne Komplikation ( $p$ 0,0092). Die genaue Berechnung der GFR mittels MDRD-Formel bestätigt somit die bereits beschriebenen Ergebnisse der Kreatininclearance. 


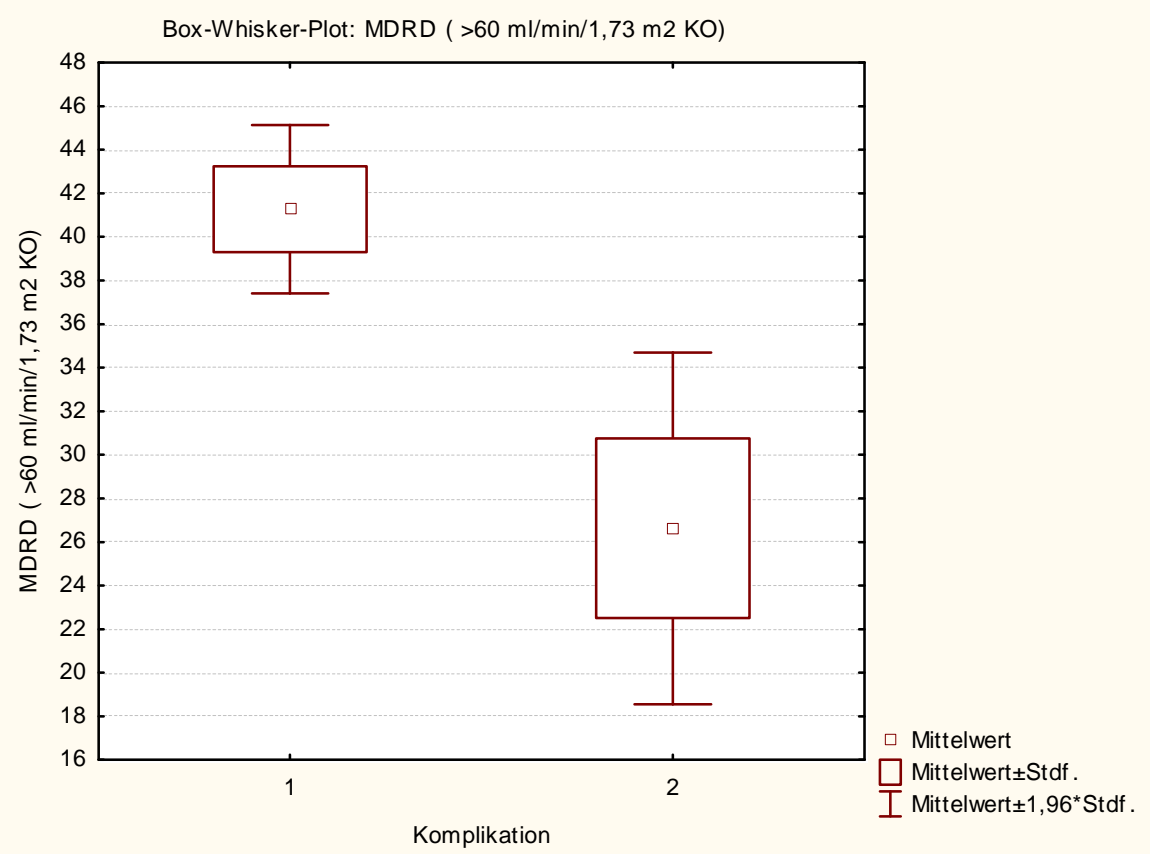

Abb. 24: Beziehung zwischen MDRD und Komplikation.

1 = keine Komplikation, 2 = Komplikation

\subsubsection{Beziehung zwischen MDRD und Komplikationsart}

1) Makrohämaturie

Die Patienten mit einer neu aufgetretenen Makrohämaturie unterschieden sich signifikant in der Höhe des MDRD-Wertes von den Patienten ohne Komplikation (p 0,0443).

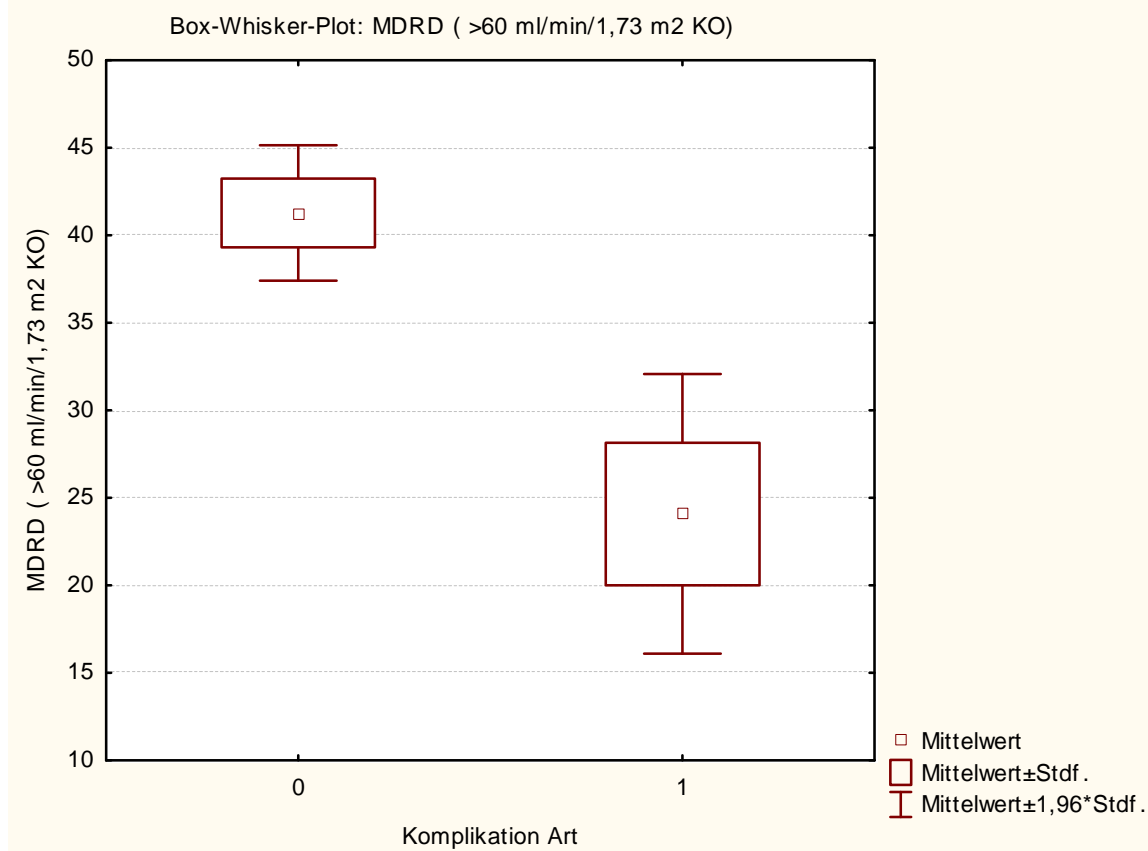

Abb. 25: Beziehung zwischen MDRD und Komplikationsart $\rightarrow$ Makrohämaturie.

$$
0=\text { keine Komplikation, } 1 \text { = Makrohämaturie }
$$




\section{2) Blutung}

Es ergab sich kein signifikanter Unterschied in der Höhe des MDRD-Wertes im Vergleich der Patienten mit Blutung nach Biopsie und der Patienten ohne Komplikation (p 0,0763).

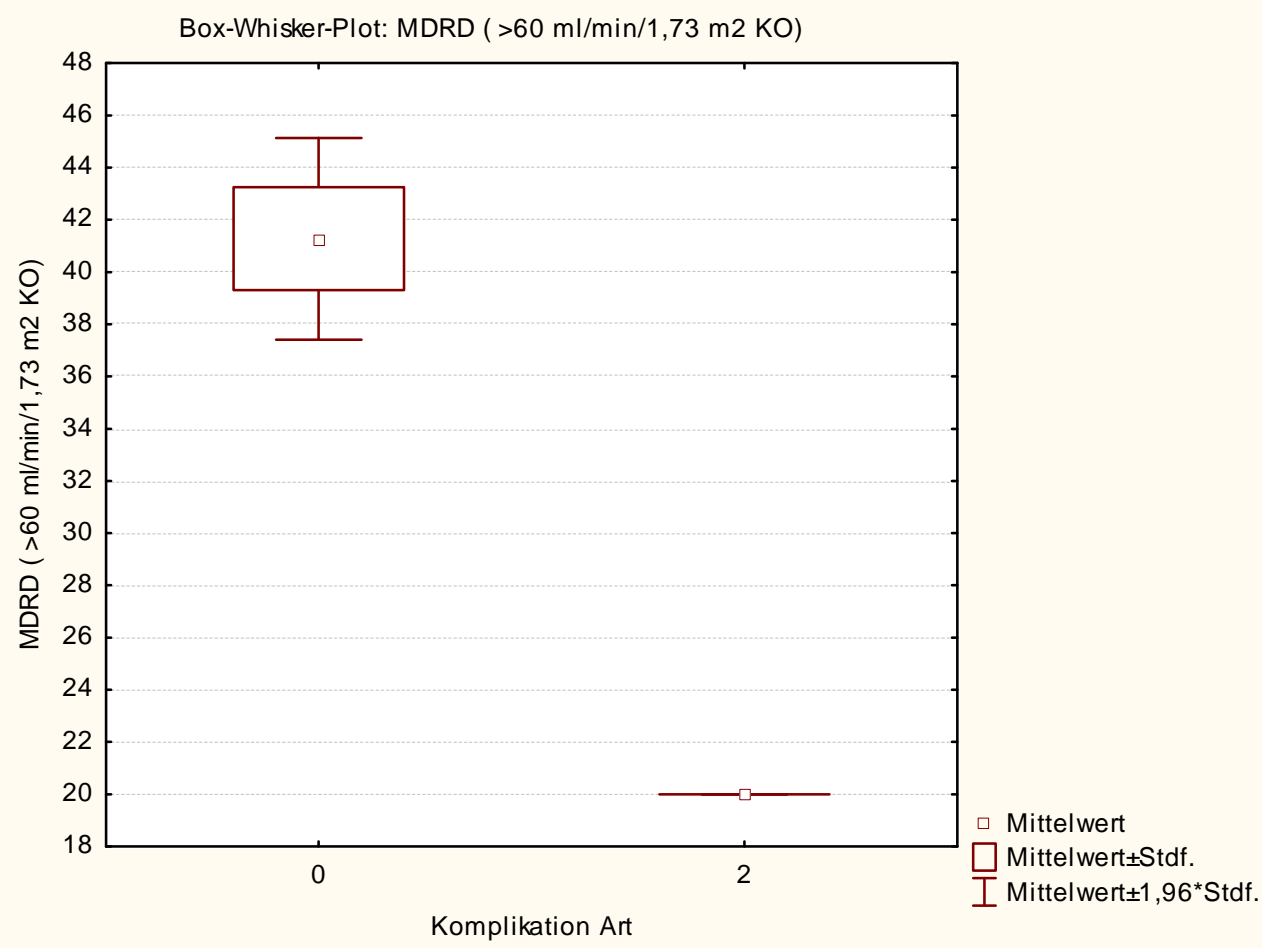

Abb. 26: Beziehung zwischen MDRD und Komplikationsart $\rightarrow$ Blutung.

$0=$ keine Komplikation, 2 = Blutung

3) Klinisch relevanter $\mathrm{Hb}$-Abfall

Die Patienten mit einem klinisch relevanten $\mathrm{Hb}$-Abfall wiesen keinen signifikanten Unterschied in der Höhe des MDRD-Wertes auf zu den Patienten ohne Komplikation ( $p$ 0,3106).

\subsubsection{Berechnung mit nominalskalierten Werten}

Aus den oben angeführten Gründen erfolgte nun eine zweite Berechnung. Dazu wurden die Patienten in verschiedene Gruppen unterteilt: 1: MDRD > 60 $\mathrm{ml} / \mathrm{min} / 1,73 \mathrm{~m}^{2} \mathrm{KO}$ (Niereninsuffizienzklassen I + II), 2: MDRD $30-60$ $\mathrm{ml} / \mathrm{min} / 1,73 \mathrm{~m}^{2} \mathrm{KO}$ (Klasse III), 3: MDRD < $30 \mathrm{ml} / \mathrm{min} / 1,73 \mathrm{~m}^{2} \mathrm{KO}$ (Klasse IV). Durchgeführt wurde jeweils der Chi-Quadrat-Test nach Pearson. 


\subsubsection{Beziehung zwischen MDRD und Hämatom}

Die Patienten mit Hämatomentwicklung zeigten keinen signifikanten Unterschied in der prozentualen Verteilung auf MDRD-Gruppen zu den Patienten ohne Hämatom ( $p$ 0,4387).

\subsubsection{Beziehung zwischen MDRD und Hb-Abfall $>1 \mathrm{~g} / \mathrm{dl}$}

Für die Patienten mit $\mathrm{Hb}$-Abfall $>1 \mathrm{~g} / \mathrm{dl}$ innerhalb der ersten 24 Stunden nach Biopsie ergab sich kein signifikanter Unterschied in der prozentualen Verteilung auf die MDRD-Gruppen zu den Patienten ohne Hb-Abfall > $1 \mathrm{~g} / \mathrm{dl}(\mathrm{p} \mathrm{0,8648)}$.

\subsubsection{Beziehung zwischen MDRD und Komplikation}

Die Patienten mit einer Komplikation nach Biopsie verteilten sich prozentual signifikant anders auf die MDRD-Gruppen als die Patienten ohne Komplikation ( $p$ 0,0338). Somit konnte das Ergebnis der ordinalskalierten Daten und auch das Ergebnis aus der Kreatininclearance bestätigt werden.

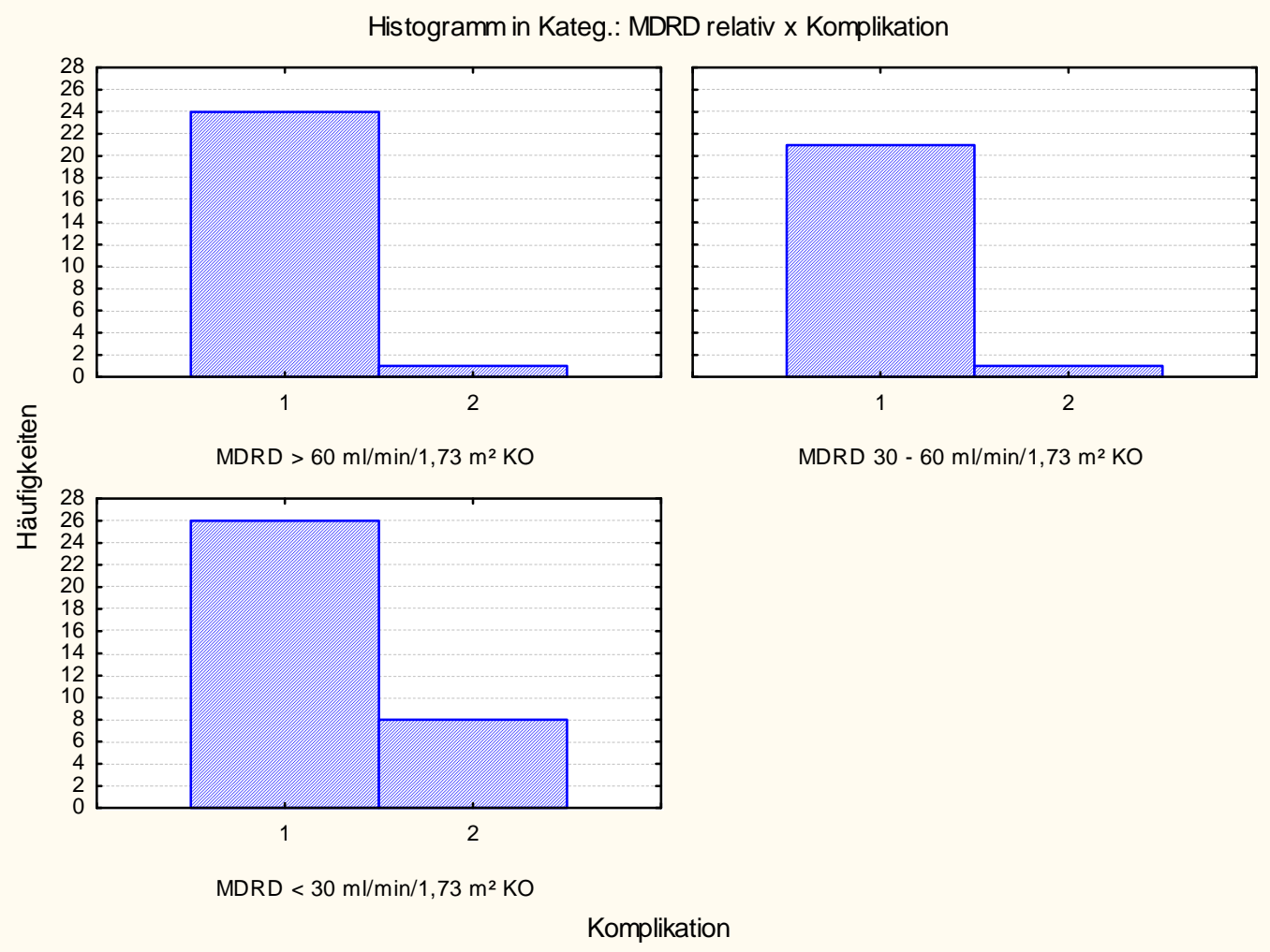

Abb. 27: Beziehung zwischen MDRD und Komplikation.

1 = keine Komplikation, 2 = Komplikation 


\subsubsection{Beziehung zwischen MDRD und Komplikationsart}

1) Makrohämaturie

Die Patienten mit einer neu aufgetretenen Makrohämaturie unterschieden sich nicht signifikant in der prozentualen Verteilung auf die MDRD-Gruppen von den Patienten ohne Komplikation ( $p$ 0,3013).

2) Blutung

Die Patienten mit einer Blutung nach Biopsie wiesen keinen signifikanten Unterschied in der prozentualen Verteilung auf die MDRD-Gruppen auf zu den Patienten ohne Komplikation ( $p$ 0,2424).

Sowohl bei Makrohämaturie als auch bei Blutung ergaben sich also keine signifikanten Werte, was die Ergebnisse der ordinalskalierten Werte für diese Parameter nicht bestätigt. Die MDRD-Formel scheint somit in Bezug auf die Prognostizierung dieser Komplikationsarten keine klinische Relevanz zu besitzen.

3) Klinisch relevanter Hb-Abfall

Es ergab sich kein signifikanter Unterschied in der prozentualen Verteilung auf die MDRD-Gruppen im Vergleich der Patienten mit klinisch relevantem $\mathrm{Hb}$ Abfall und der Patienten ohne Komplikation ( $p$ 0,3193).

\subsection{Sonstige Erkrankungen als Parameter zur Evaluation von Risiken}

Erfasst wurden arterielle Hypertonie, Diabetes mellitus und eine stattgehabte Nierentransplantation. Durchgeführt wurde jeweils der Chi-Quadrat-Test nach Pearson.

\subsubsection{Arterielle Hypertonie}

\subsubsection{Beziehung zwischen arterieller Hypertonie und Hämatom}

Die Patienten mit Hämatomentwicklung wiesen keinen signifikanten Unterschied in der prozentualen Verteilung auf die Gruppen mit und ohne arterielle Hypertonie auf zu den Patienten ohne Hämatom ( $p$ 0,9702). 


\subsubsection{Beziehung zwischen arterieller Hypertonie und $\mathrm{Hb}-A b f a l l ~>1 \mathrm{~g} / \mathrm{dl}$}

Die Patienten mit Hb-Abfall > $1 \mathrm{~g} / \mathrm{dl}$ innerhalb der ersten 24 Stunden nach Biopsie zeigten keinen signifikanten Unterschied in der prozentualen Verteilung auf die Gruppen mit und ohne arterielle Hypertonie zu den Patienten ohne Hb-Abfall > 1 $\mathrm{g} / \mathrm{dl}(\mathrm{p} 0,7338)$.

\subsubsection{Beziehung zwischen arterieller Hypertonie und Komplikation}

Die Patienten mit einer Komplikation nach Biopsie unterschieden sich nicht signifikant in der prozentualen Verteilung auf die Gruppen mit und ohne arterielle Hypertonie von den Patienten ohne Komplikation ( $p$ 0,5647).

\subsubsection{Beziehung zwischen arterieller Hypertonie und Komplikationsart}

1) Makrohämaturie

Für die Patienten mit einer neu aufgetretenen Makrohämaturie ergab sich kein signifikanter Unterschied in der prozentualen Verteilung auf die Gruppen mit und ohne arterielle Hypertonie zu den Patienten ohne Komplikation ( $p$ 0,3949).

2) Blutung

Die Patienten mit einer Blutung nach Biopsie wiesen keinen signifikanten Unterschied in der prozentualen Verteilung auf die Gruppen mit und ohne arterielle Hypertonie auf zu den Patienten ohne Komplikation ( $p 0,2990)$.

3) Klinisch relevanter Hb-Abfall

Im Vergleich der Patienten mit einem klinisch relevanten Hb-Abfall in Bezug auf eine arterielle Hypertonie und der Patienten ohne Komplikation ergab sich kein signifikanter Unterschied in der prozentualen Verteilung auf die Gruppen mit und ohne arteriellen Bluthochdruck ( $p$ 0,3949).

\subsubsection{Diabetes mellitus}

\subsubsection{Beziehung zwischen Diabetes mellitus und Hämatom}

Für die Gefahr einer Hämatomentwicklung zeigte sich kein signifikanter Unterschied in der prozentualen Verteilung auf die Gruppen der Patienten mit und ohne Diabetes mellitus ( $p$ 0,8955). 


\subsubsection{Beziehung zwischen Diabetes mellitus und Hb-Abfall $>1 \mathrm{~g} / \mathrm{dl}$}

Die Patienten mit Hb-Abfall $>1 \mathrm{~g} / \mathrm{dl}$ innerhalb der ersten 24 Stunden nach Biopsie wiesen keinen signifikanten Unterschied in der prozentualen Verteilung auf die Gruppen mit und ohne Diabetes mellitus auf zu den Patienten ohne $\mathrm{Hb}$ Abfall $>1 \mathrm{~g} / \mathrm{dl}(\mathrm{p} 0,1387)$.

\subsubsection{Beziehung zwischen Diabetes mellitus und Komplikation}

Die Patienten mit einer Komplikation nach Biopsie unterschieden sich nicht signifikant in der prozentualen Verteilung auf die Gruppen mit und ohne Diabetes mellitus von den Patienten ohne Komplikation ( $p$ 0,8680).

\subsubsection{Beziehung zwischen Diabetes mellitus und Komplikationsart}

1) Makrohämaturie

Die Patienten mit einer neu aufgetretenen Makrohämaturie zeigten keinen signifikanten Unterschied in der prozentualen Verteilung auf die Gruppen mit und ohne Diabetes mellitus zu den Patienten ohne Komplikation ( $p 0,2992)$.

2) Blutung

Für die Patienten mit einer Blutung nach Biopsie ergab sich kein signifikanter Unterschied in der prozentualen Verteilung auf die Gruppen mit und ohne Diabetes mellitus zu den Patienten ohne Komplikation ( $p$ 0,4261).

3) Klinisch relevanter $\mathrm{Hb}$-Abfall

Die Patienten mit einem klinisch relevanten $\mathrm{Hb}$-Abfall wiesen keinen signifikanten Unterschied in der prozentualen Verteilung auf die Gruppen mit und ohne Diabetes mellitus auf zu den Patienten ohne Komplikation ( $p$ 0,1601).

\subsubsection{Transplantatniere}

\subsubsection{Beziehung zwischen Transplantatniere und Hämatom}

Die Patienten mit Hämatomentwicklung unterschieden sich nicht signifikant in der prozentualen Verteilung auf die Gruppen mit und ohne Transplantatniere von den Patienten ohne Hämatom ( $p$,6998). 


\subsubsection{Beziehung zwischen Transplantatniere und $\mathrm{Hb}-A b f a l l ~>1 \mathrm{~g} / \mathrm{dl}$}

Die Patienten mit Hb-Abfall $>1 \mathrm{~g} / \mathrm{dl}$ innerhalb der ersten 24 Stunden nach Biopsie zeigten keinen signifikanten Unterschied in der prozentualen Verteilung auf die Gruppen mit und ohne Transplantatniere zu den Patienten ohne $\mathrm{Hb}$ Abfall $>1 \mathrm{~g} / \mathrm{dl}(\mathrm{p} \mathrm{0,1966)}$.

\subsubsection{Beziehung zwischen Transplantatniere und Komplikation}

Die Patienten mit einer Komplikation nach Biopsie wiesen keinen signifikanten Unterschied in der prozentualen Verteilung auf die Gruppen mit und ohne Transplantatniere auf zu den Patienten ohne Komplikation (p 0,9200).

\subsubsection{Beziehung zwischen Transplantatniere und Komplikationsart}

1) Makrohämaturie

Für die Patienten mit einer neu aufgetretenen Makrohämaturie zeigte sich kein signifikanter Unterschied in der prozentualen Verteilung auf die Gruppen mit und ohne Transplantatniere zu den Patienten ohne Komplikation ( $p$ 0,3188).

2) Blutung

Es ergab sich kein signifikanter Unterschied in der prozentualen Verteilung auf die Gruppen mit und ohne Transplantatniere im Vergleich der Patienten mit einer Blutung nach Biopsie und der Patienten ohne Komplikation ( $p$ 0,3503).

3) Klinisch relevanter $\mathrm{Hb}$-Abfall

Die Patienten mit einem klinisch relevanten $\mathrm{Hb}$-Abfall zeigten keinen signifikanten Unterschied in der prozentualen Verteilung auf die Gruppen mit und ohne Transplantatniere zu den Patienten ohne Komplikation ( $p$ 0,9118).

\subsection{Die nephrologische Diagnose als Parameter zur Evaluation von Risiken}

\subsubsection{Beziehung zwischen nephrologischer Diagnose und Hämatom}

Die Patienten mit einem Hämatom wiesen keinen signifikanten Unterschied in der prozentualen Verteilung auf die Gruppen mit den unterschiedlichen nephrologischen Diagnosen auf zu den Patienten ohne Hämatom ( $p 0,2313$ ). 


\subsubsection{Beziehung zwischen nephrologischer Diagnose und Hb-Abfall $>1 \mathrm{~g} / \mathrm{dl}$}

Die Patienten mit einem Hb-Abfall $>1 \mathrm{~g} / \mathrm{dl}$ innerhalb der ersten 24 Stunden nach Biopsie unterschieden sich nicht signifikant in der prozentualen Verteilung auf die Gruppen mit den unterschiedlichen nephrologischen Diagnosen von den Patienten ohne Hämatom ( $p 0,4346)$.

\subsubsection{Beziehung zwischen nephrologischer Diagnose und Komplikation}

Für die Patienten mit einer Komplikation ergab sich kein signifikanter Unterschied in der prozentualen Verteilung auf die Gruppen mit den unterschiedlichen nephrologischen Diagnosen im Vergleich zu den Patienten ohne Komplikation ( $p$ $0,5544)$.

\subsubsection{Beziehung zwischen nephrologischer Diagnose und Komplikationsart}

1) Makrohämaturie

Es konnte kein signifikanter Unterschied nachgewiesen werden bei der prozentualen Verteilung auf die Gruppen mit den unterschiedlichen nephrologischen Diagnosen im Vergleich der Patienten mit einer Makrohämaturie und der Patienten ohne Komplikation ( $p$ 0,8786).

2) Blutung

Die Patienten mit einer Blutung nach Biopsie zeigten keinen signifikanten Unterschied in der prozentualen Verteilung auf die Gruppen mit den unterschiedlichen nephrologischen Diagnosen zu den Patienten ohne Komplikation ( $p$ 0,7747).

3) Klinisch relevanter $\mathrm{Hb}$-Abfall

Die Patienten mit einem klinisch relevanten $\mathrm{Hb}$-Abfall wiesen keinen signifikanten Unterschied in der prozentualen Verteilung auf die Gruppen mit den unterschiedlichen nephrologischen Diagnosen auf zu den Patienten ohne Komplikation (p 0,8786). 


\subsection{Komplikationsrate bei Eigenniere und nach Nierentransplantation}

\subsubsection{Komplikationsrate bei Eigenniere}

$\mathrm{Da}$ in die Berechnung der bisher aufgeführten Ergebnisse immer Patienten mit und ohne Nierentransplantation mit eingingen, wurde für die Komplikationsrate nochmals eine zusätzliche Berechnung gemacht. Hierfür wurden nur Patienten mit Eigenniere berücksichtigt. Als Komplikation galten alle symptomatischen Komplikationen nach Biopsie, das heißt Makrohämaturie, Blutung und klinisch relevanter Hb-Abfall. Zunächst soll eine Übersicht über die Ergebnisse in Form von Tabellen gegeben werden: 


\begin{tabular}{|c|c|c|c|}
\hline Parameter & Keine Komplikation & Komplikation & p-Wert \\
\hline PFA & & & 0,9004 \\
\hline 1 zu niedrig & $6(8,96 \%)$ & $1(10,00 \%)$ & \\
\hline 2 normal & $45(67,16 \%)$ & $6(60,00 \%)$ & \\
\hline 3 zu hoch & $16(23,88 \%)$ & $3(30,00 \%)$ & \\
\hline Geschlecht & & & 0,3785 \\
\hline 1 männlich & $37(55,22 \%)$ & $7(70,00 \%)$ & \\
\hline 2 weiblich & $30(44,78 \%)$ & $3(30,00 \%)$ & \\
\hline Nadeldicke & & & 0,6818 \\
\hline $114 G$ & $34(58,62 \%)$ & $6(60,00 \%)$ & \\
\hline $216 G$ & $20(34,48 \%)$ & $4(40,00 \%)$ & \\
\hline $318 \mathrm{G}$ & $4(6,90 \%)$ & $0(0,00 \%)$ & \\
\hline Quick & & & 0,7105 \\
\hline $1<70 \%$ & $2(3,13 \%)$ & $0(0,00 \%)$ & \\
\hline $270-120 \%$ & $41(64,06 \%)$ & $5(55,56 \%)$ & \\
\hline $3>120 \%$ & $21(32,81 \%)$ & $4(44,44 \%)$ & \\
\hline MDRD & & & 0,0914 \\
\hline $1>60 \mathrm{ml} / \mathrm{min} / 1,73 \mathrm{~m}^{2} \mathrm{KO}$ & $20(36,36 \%)$ & $1(12,50 \%)$ & \\
\hline $230-60 \mathrm{ml} / \mathrm{min} / 1,73 \mathrm{~m}^{2} \mathrm{KO}$ & $16(29,09 \%)$ & $1(12,50 \%)$ & \\
\hline $3<30 \mathrm{ml} / \mathrm{min} / 1,73 \mathrm{~m}^{2} \mathrm{KO}$ & $19(34,55 \%)$ & $6(75,00 \%)$ & \\
\hline Arterielle Hypertonie & & & 0,6463 \\
\hline $1 \mathrm{ja}$ & $35(52,24 \%)$ & $6(60,00 \%)$ & \\
\hline 2 nein & $32(47,76 \%)$ & $4(40,00 \%)$ & \\
\hline Diabetes mellitus & & & 0,7779 \\
\hline $1 \mathrm{ja}$ & $11(16,42 \%)$ & $2(20,00 \%)$ & \\
\hline 2 nein & $56(83,58 \%)$ & $8(80,00 \%)$ & \\
\hline Nephrologische Diagnose & & & 0,7836 \\
\hline 1 Nephrosklerose & $8(11,94 \%)$ & $3(30,00 \%)$ & \\
\hline 2 FSGS & $15(22,39 \%)$ & $1(10,00 \%)$ & \\
\hline $3 \lg A-N e p h r i t i s$ & $17(25,37 \%)$ & $2(20,00 \%)$ & \\
\hline 4 Lupusnephritis & $6(8,96 \%)$ & $1(10,00 \%)$ & \\
\hline 5 membranoproliferative GN & $2(2,99 \%)$ & $0(0,00 \%)$ & \\
\hline 6 membranöse GN & $3(4,48 \%)$ & $0(0,00 \%)$ & \\
\hline 7 Transplantat - Glomerulopathie & $1(1,49 \%)$ & $0(0,00 \%)$ & \\
\hline 8 minimal change GN & $4(5,97 \%)$ & $0(0,00 \%)$ & \\
\hline 9 Diabetische Nephropathie & $1(1,49 \%)$ & $0(0,00 \%)$ & \\
\hline 10 Andere & $10(14,93 \%)$ & $3(30,00 \%)$ & \\
\hline
\end{tabular}

Tab. 13 Nominalskalierte Daten in Bezug auf Komplikationsrate, nur Eigenniere 


\begin{tabular}{|c|c|c|c|c|c|}
\hline \multirow[t]{2}{*}{ Parameter } & \multicolumn{2}{|c|}{ Keine Komplikation } & \multicolumn{2}{|l|}{ Komplikation } & \multirow[t]{2}{*}{ p-Wert } \\
\hline & $\mathrm{MW}+/-\mathrm{SF}$ & (N) & MW +/-SF & (N) & \\
\hline PFA & $150,04+/-9,10$ & (67) & $153,60+/-21,03$ & $(10)$ & 0,8870 \\
\hline Alter & $49,25+/-1,90$ & (67) & $52,60+/-3,62$ & (10) & 0,5161 \\
\hline Stat. Aufenthalt & $14,45+/-1,87$ & (65) & $16,60+/-3,66$ & (10) & 0,6674 \\
\hline Hämoglobin & $12,29+/-0,23$ & (65) & $11,08+/-0,56$ & $(10)$ & 0,0556 \\
\hline Quick & $109,17+/-2,24$ & $(64)$ & $115,78+/-4,77$ & (9) & 0,2942 \\
\hline PTT & $28,15+/-0,49$ & (65) & $26,02+/-1,20$ & (10) & 0,1154 \\
\hline Kreatinin & $2,07+/-0,17$ & (67) & $4,92+/-1,00$ & (10) & $<0,0001$ \\
\hline Kreatininclearance & $61,28+/-6,10$ & (42) & $37,12+/-19,15$ & (6) & 0,1774 \\
\hline MDRD & $41,86+/-2,30$ & (55) & $28,29+/-5,03$ & (8) & 0,0364 \\
\hline
\end{tabular}

Tab. 14 Ordinalskalierte Daten in Bezug auf Komplikationsrate, nur Eigenniere

3.16.2 Komplikationsrate nach Nierentransplantation

Auch die Gruppe der nierentransplantierten Patienten wurde nochmals untersucht auf den Bezug zur Komplikationsrate. Auch hier wurden alle symptomatischen Komplikationen (Makrohämaturie, Blutung, klinisch relevanter $\mathrm{Hb}$-Abfall) in die Bewertung mit einbezogen. Im Folgenden werden die Ergebnisse in Form von Tabellen dargestellt: 


\begin{tabular}{|c|c|c|c|}
\hline Parameter & Keine Komplikation & Komplikation & p-Wert \\
\hline PFA & & & 0,7223 \\
\hline 1 zu niedrig & $3(15,79 \%)$ & $0(0,00 \%)$ & \\
\hline 2 normal & $12(63,16 \%)$ & $2(66,67 \%)$ & \\
\hline 3 zu hoch & $4(21,05 \%)$ & $1(33,33 \%)$ & \\
\hline Geschlecht & & & 0,9065 \\
\hline 1 männlich & $12(63,16 \%)$ & $2(66,67 \%)$ & \\
\hline 2 weiblich & $7(36,84 \%)$ & $1(33,33 \%)$ & \\
\hline Nadeldicke & & & 0,2738 \\
\hline $114 G$ & $5(31,25 \%)$ & $0(0,00 \%)$ & \\
\hline $216 G$ & $8(50,00 \%)$ & $3(100,00 \%)$ & \\
\hline $318 \mathrm{G}$ & $3(18,75 \%)$ & $0(0,00 \%)$ & \\
\hline Quick & & & 0,9022 \\
\hline $1<70 \%$ & $1(5,26 \%)$ & $0(0,00 \%)$ & \\
\hline $270-120 \%$ & $13(68,42 \%)$ & $2(66,67 \%)$ & \\
\hline $3>120 \%$ & $5(26,32 \%)$ & $1(33,33 \%)$ & \\
\hline MDRD & & & 0,3652 \\
\hline $1>60 \mathrm{ml} / \mathrm{min} / 1,73 \mathrm{~m}^{2} \mathrm{KO}$ & $3(20,00 \%)$ & $0(0,00 \%)$ & \\
\hline $230-60 \mathrm{ml} / \mathrm{min} / 1,73 \mathrm{~m}^{2} \mathrm{KO}$ & $5(33,33 \%)$ & $0(0,00 \%)$ & \\
\hline $3<30 \mathrm{ml} / \mathrm{min} / 1,73 \mathrm{~m}^{2} \mathrm{KO}$ & $7(46,67 \%)$ & $2(100,00 \%)$ & \\
\hline Arterielle Hypertonie & & & 0,6842 \\
\hline $1 \mathrm{ja}$ & $18(94,74 \%)$ & $3(100,00 \%)$ & \\
\hline 2 nein & $1(5,26 \%)$ & $0(0,00 \%)$ & \\
\hline Diabetes mellitus & & & 0,3796 \\
\hline $1 \mathrm{ja}$ & $4(21,05 \%)$ & $0(0,00 \%)$ & \\
\hline 2 nein & $15(78,95 \%)$ & $3(100,00 \%)$ & \\
\hline Nephrologische Diagnose & & & 0,5362 \\
\hline 1 Nephrosklerose & $4(21,05 \%)$ & $2(66,67 \%)$ & \\
\hline 2 FSGS & $3(15,79 \%)$ & $0(0,00 \%)$ & \\
\hline $3 \lg A-N e p h r i t i s$ & $0(0,00 \%)$ & $0(0,00 \%)$ & \\
\hline 4 Lupusnephritis & $0(0,00 \%)$ & $0(0,00 \%)$ & \\
\hline 5 membranoproliferative GN & $0(0,00 \%)$ & $0(0,00 \%)$ & \\
\hline 6 membranöse GN & $0(0,00 \%)$ & $0(0,00 \%)$ & \\
\hline 7 Transplantat - Glomerulopathie & $3(15,79 \%)$ & $0(0,00 \%)$ & \\
\hline 8 minimal change GN & $0(0,00 \%)$ & $0(0,00 \%)$ & \\
\hline 9 Diabetische Nephropathie & $1(5,26 \%)$ & $0(0,00 \%)$ & \\
\hline 10 Andere & $8(42,11 \%)$ & $1(33,33 \%)$ & \\
\hline
\end{tabular}

Tab. 15 Nominalskalierte Daten in Bezug auf Komplikationsrate, nur Nierentransplantate 


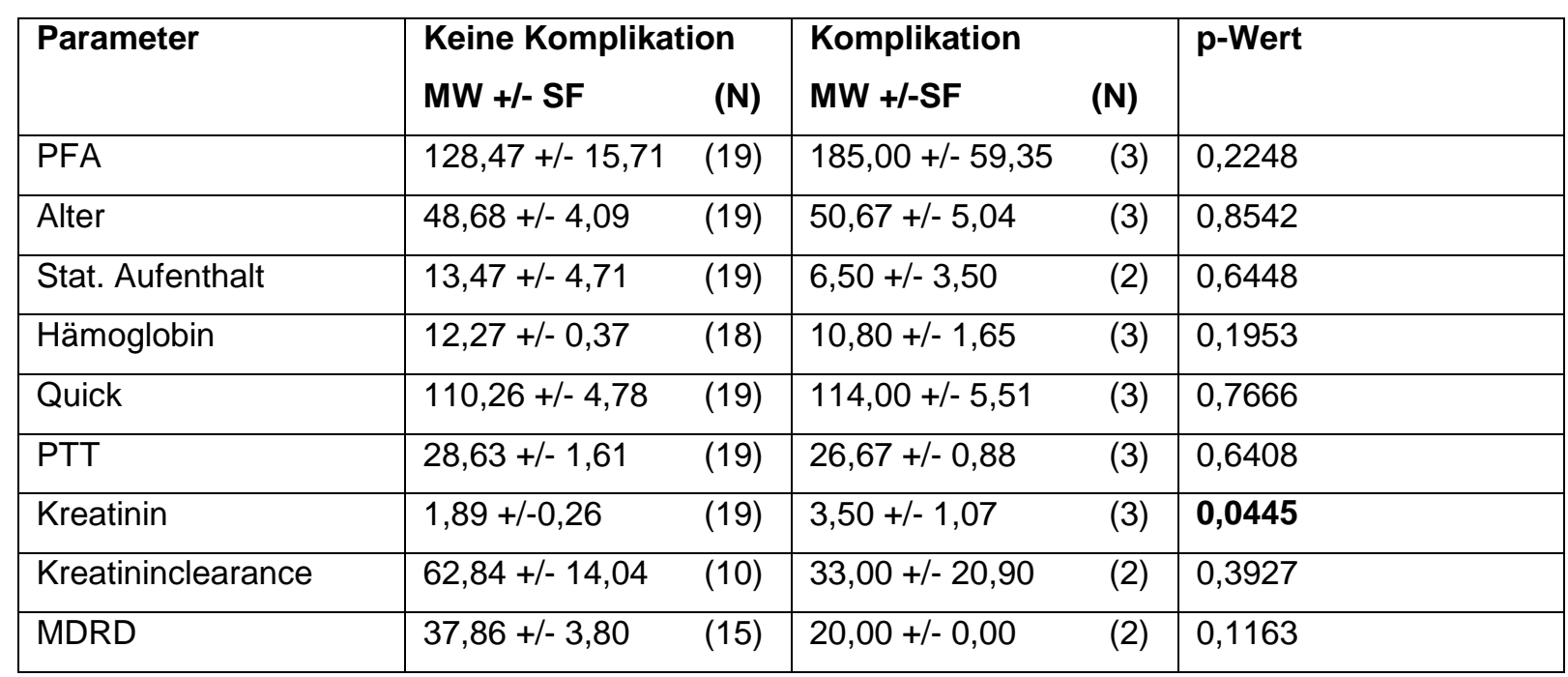

Tab. 16 Ordinalskalierte Daten in Bezug auf Komplikationsrate, nur Nierentransplantate

Die beiden Gruppen der nierentransplantierten Patienten und der Patienten mit Eigenniere unterscheiden sich nur unwesentlich in den p-Werten. Auch die pWerte unter Berücksichtigung aller Nierenbiopsien, transplantiert und Eigenniere, unterscheiden sich kaum von den $\mathrm{p}$-Werten mit Unterscheidung der beiden Gruppen. Die Nierenfunktion, insbesondere der Kreatininwert ist in allen Berechnungen in Bezug auf die Komplikationsrate der einzige wirklich erkennbare Risikofaktor für Komplikationen. MDRD und Kreatininclearance sind sowohl im gesamten Patientenkollektiv als auch in der Unterscheidung zwischen Nierentransplantat und Eigenniere nur unzuverlässige und somit nicht klinisch verwendbare Parameter zur Detektion des individuellen Risikos für Komplikationen. 


\section{Diskussion}

\subsection{Die Komplikationsrate}

In unserer retrospektiven Analyse zeigte sich eine Komplikationsrate von 13\%. Als Komplikation wurde eine Makrohämaturie, eine Blutung oder ein klinisch relevanter $\mathrm{Hb}$-Abfall nach Biopsie gewertet, der die Bereitstellung oder Gabe von Erythrozytenkonzentraten erforderte. $5 \%$ der Patienten hatten eine Makrohämaturie, 5\% einen klinisch signifikanten $\mathrm{Hb}$-Abfall und 3\% der Patienten hatten eine Blutung. Ein Patient mit einer großen retroperitonealen Blutung musste nach der Biopsie operiert werden. Bei den Patienten mit einer Makrohämaturie war keine Intervention nötig.

Bei 19\% der Patienten konnte nach der Biopsie sonographisch ein perirenales Hämatom nachgewiesen werden und $41 \%$ der Patienten zeigten nach der Biopsie einen $\mathrm{Hb}$-Abfall $>1 \mathrm{~g} / \mathrm{dl}$. Die meisten dieser Patienten zeigten aber einen blanden Verlauf nach der Biopsie und wiesen keine Komplikation auf.

Die Komplikationsrate deckt sich weitestgehend mit den Komplikationsraten aus anderen Studien, wobei die Anzahl schwerer Komplikationen mit sofortiger Intervention nur $1 \%$ betrug und vergleichsweise gering war. Es gab keine auf die Biopsie zurückführbare Todesfälle.

Diese relativ geringe Komplikationsrate, insbesondere bezogen auf schwere Komplikationen, zeigt, dass die perkutane Nierenbiopsie heute ein relativ sicheres Verfahren und bei klarer Indikationsstellung und der genauen Beachtung von Kontraindikationen diagnostisch von sehr hohem Wert im klinischen Alltag sein kann.

Diese Erkenntnis deckt sich mit anderen Studien (Whittier und Korbet 2004 b, Khajehdehi et al. 1999, Gonzalez-Michaca et al. 2000).

Preda et al. hatten in ihrer Studie mit 515 Patienten eine Komplikationsrate von 12,2\%. 2,7\% der Fälle hatten schwere, 9,5\% leichte Komplikationen (Preda et al. 2003). Diese Daten decken sich weitestgehend mit den Daten aus unserer Studie.

Chen et al. konnten in ihrer Studie mit 1000 Patienten mit einer perkutanen Nierenbiopsie bei $7,4 \%$ der Patienten eine Makrohämaturie, bei $4,4 \%$ ein 
perirenales Hämatom und bei $0,6 \%$ schwere Blutungskomplikationen nachweisen (Chen et al. 2000).

Prakash et al. hatten in ihrer Studie mit 305 Patienten eine Komplikationsrate von $11,8 \%$ mit Makrohämaturie, $0,98 \%$ mit der Notwendigkeit von Erythrozytenkonzentraten und $1,6 \%$ mit perirenalem Hämatom (Prakash et al. 1994).

Obwohl das Risiko bei Nierenbiopsien verhältnismäßig gering ist, gibt es noch keine Möglichkeit, das individuelle Blutungsrisiko zufrieden stellend zu prognostizieren.

\subsection{PFA- $100^{\mathrm{TM}}$ als Risikofaktor für Komplikationen}

Wir untersuchten nun zusätzlich den Einfluss des PFA-100 ${ }^{\mathrm{TM}}$-Wertes auf das Blutungsrisiko, da diverse Studien bereits eine sehr hohe Sensitivität des PFA$100^{\mathrm{TM}}$-Wertes bei der Detektion von Thrombozytenfunktionsstörungen, wie zum Beispiel bei der von-Willebrand-Krankheit, zeigten (Fressinaud et al. 1998, Favaloro et al. 1999, Favaloro et al. 2001).

Leider konnten wir keinen signifikanten Einfluss des PFA-100 ${ }^{\mathrm{TM}}$-Wertes in Bezug auf das Risiko von Hämatomentstehung nach Biopsie, einem Hb-Abfall > $1 \mathrm{~g} / \mathrm{dl}$ innerhalb von 24 Stunden nach Biopsie oder einer Komplikation nachweisen. Allerdings zeigte sich eine Tendenz von erhöhten PFA-100 ${ }^{\mathrm{TM}}$-Werten bei Patienten mit einem $\mathrm{Hb}$-Abfall $>1 \mathrm{~g} / \mathrm{dl}$ innerhalb von 24 Stunden nach Biopsie. Dieses Ergebnis ist zwar nicht ausreichend, um ein individuelles Risiko anzugeben, zeigt aber, dass Patienten mit einem erhöhten PFA $-100^{\mathrm{TM}}$-Wert tendenziell einen höheren $\mathrm{Hb}$-Abfall hatten als Patienten mit einem normalen PFA-100 ${ }^{\mathrm{TM}}$-Wert. Allerdings ist diese Tendenz unsicher, da Spät- und Nachtbestimmungen des $\mathrm{Hb}$-Wertes oft niedrigere Werte angeben, als tatsächlich vorliegen und so auch die Gefahr falsch positiver Ergebnisse bei der Beobachtung des $\mathrm{Hb}$-Wertes im Verlauf gegeben ist. Zudem konnte dieses Ergebnis durch die nominal skalierten Daten nicht bestätigt werden. Hier zeigte sich für keinen der untersuchten Parameter ein signifikantes Ergebnis.

Durch den großen Unterschied in den Stichprobenumfängen, vor allem bei der Aufteilung nach den Komplikationsarten, kann es zu falsch negativen 
Ergebnissen gekommen sein. Damit wäre ein Zusammenhang zwischen PFA$100^{\mathrm{TM}}$ und bestimmten Komplikationsarten eventuell statistisch nicht entdeckt worden. Trotzdem bleibt die klinische Relevanz von PFA-100 ${ }^{\mathrm{TM}}$ bei perkutaner Nierenbiopsie gering, da auch kein Zusammenhang mit der allgemeinen Komplikationsrate nachgewiesen werden konnte.

Slaughter et al. konnten in ihrer Studie nachweisen, dass der PFA-100 ${ }^{\mathrm{TM}}$-Wert nicht mit der Blutungsneigung nach herzchirurgischen Eingriffen korrelierte, dass allerdings Patienten mit exzessiven postoperativen Blutungen einen wesentlich höheren PFA-100 ${ }^{\mathrm{TM}}$-Wert hatten als Patienten mit geringfügigeren Blutungen (Slaughter et al. 2001).

Auch Forestier et al. kamen in ihrer Studie mit Bypass operierten Patienten zu dem Schluss, dass die Bestimmung von PFA-100 ${ }^{\mathrm{TM}}$ zur Detektion von postoperativen Komplikationsrisiken nur bei Patienten mit einem erhöhten Risiko von Bedeutung ist (Forestier et al. 2002).

Ebenso konnten Wahba et al. zeigen, dass PFA $-100^{\mathrm{TM}}$ zwar mit dem Blutverlust nach herzchirurgischen Eingriffen korreliert, die Anwendung in der Praxis allerdings aufgrund hoher Variabilität des PFA- $100^{\mathrm{TM}}$-Wertes sehr begrenzt ist (Wahba et al. 1998).

In einer von Fattobrutto et al. durchgeführten Studie zur Bedeutung von PFA$100^{\mathrm{TM}}$ zur Detektion von Blutverlust nach kardiopulmonalem Bypass zeigte sich, dass anhand von PFA-100 ${ }^{\mathrm{TM}}$ nicht unterschieden werden konnte zwischen Patienten mit niedrigem Risiko für eine Nachblutung und Patienten mit starken Nachblutungen (Fattobrutto et al. 2003).

In einer Dissertationsarbeit der Rheinisch-Westfälischen Technischen Hochschule Aachen wurde untersucht, ob PFA-100 ${ }^{\mathrm{TM}}$ geeignet ist zur Prognostizierung periund postoperativer Blutungskomplikationen bei gynäkologischen Operationen. Es konnte dabei keine Signifikanz nachgewiesen werden (Metzler 2001).

In einer 2006 veröffentlichten Studie untersuchten Blanco et al. die Spezifität von PFA-100 ${ }^{\mathrm{TM}}$ bei der Bestimmung der Blutungszeit im Vergleich zum Ivy-Test. Hierbei ergab sich, dass der PFA-100 ${ }^{\mathrm{TM}}$-Wert eine wesentlich höhere Spezifität bei der Bestimmung der Blutungszeit ergab als der Ivy-Test (Blanco et al. 2006). Im Vergleich zwischen PFA-100 ${ }^{\mathrm{TM}}$ und Blutungszeit bei Patienten mit mukokutanen Blutungen konnte allerdings bei beiden Faktoren keine hohe 
Sensitivität und kein signifikanter Unterschied in der Detektion solcher Blutungen nachgewiesen werden (Quiroga et al. 2004).

Ebenso konnten beide Faktoren in einer weiteren Studie keine ausreichende Sensitivität zur Detektion von Hämostasestörungen aufweisen (Podda et al. 2007).

Auch Hayward et al. kamen zu dem Schluss, dass PFA-100 ${ }^{\mathrm{TM}}$ eine zu geringe Sensitivität besitzt, um als Screening Parameter bei Thrombozytenfunktionsstörungen Verwendung zu finden (Hayward et al. 2006). Obwohl noch kein Hinweis darauf erbracht werden konnte, dass PFA-100 ${ }^{\mathrm{TM}}$ dazu beitragen kann, Risikopatienten für Blutungskomplikationen bei chirurgischen Eingriffen besser herauszufinden, zeigten Ortel et al., dass viele Patienten in ihrer Studie mit einer verlängerten Verschlusszeit bei Kollagen/ADP Störungen in der primären Hämostase aufweisen, die eventuell zu einem erhöhten Blutungsrisiko bei Eingriffen führen könnten (Ortel et al. 2000).

Zusätzlich zu der Relevanz von PFA-100 ${ }^{\mathrm{TM}}$ bei Blutungskomplikationen untersuchten wir in unserer Studie den Einfluss weiterer Faktoren auf das Blutungsrisiko nach Nierenbiopsien.

\subsection{Das Geschlecht als Risikofaktor für Komplikationen}

Zunächst wurde der Einfluss des Geschlechtes untersucht. Die p-Werte für die untersuchten Parameter Hämatom, Hb-Abfall, Komplikation und Komplikationsarten lagen außerhalb des Signifikanzbereiches. Lediglich für den klinisch relevanten $\mathrm{Hb}$-Abfall konnte eine Tendenz zum männlichen Geschlecht aufgezeigt werden ( $p$-Wert 0,0509). 100\% der Patienten mit einem klinisch relevanten $\mathrm{Hb}$-Abfall waren in unserer Studie männlich. Dieses Ergebnis ist allerdings nicht sehr aussagekräftig, da lediglich fünf Patienten einen klinisch relevanten $\mathrm{Hb}$-Abfall aufwiesen und der Stichprobenumfang somit sehr klein ist. Deshalb konnten wir kein sicher erhöhtes Risiko für Blutungskomplikationen abhängig vom Geschlecht nachweisen.

Bereits in früheren Studien wurde geprüft, ob es geschlechtsabhängig ein höheres Risiko für Blutungskomplikationen bei Nierenbiopsien gibt. In einer 2001 veröffentlichten Studie ergaben sich Hinweise darauf, dass das Blutungsrisiko bei 
Männern höher ist als bei Frauen, was sich in der logistischen Regression allerdings nicht durch Signifikanz belegen ließ (Stiles et al. 2001).

In einer weiteren Studie aus dem Jahre 2004 konnte kein Zusammenhang zwischen dem Geschlecht und dem Blutungsrisiko gezeigt werden (Whittier und Korbet 2004 b).

Manno et al. veröffentlichten 2004 eine Studie, in der Frauen signifikant häufiger Komplikationen aufwiesen (Manno et al. 2004).

\subsection{Das Alter als Risikofaktor für Komplikationen}

Manno et al. konnten in ihrer Studie außerdem zeigen, dass ein jüngeres Alter mit einer höheren Komplikationsrate einhergeht. Auch dieser Parameter wurde in unserer Studie untersucht. In Bezug auf einen Hb-Abfall > $1 \mathrm{~g} / \mathrm{dl}$ innerhalb von 24 Stunden nach Biopsie, die Komplikationsrate und die einzelnen Komplikationsarten konnte dieses Ergebnis von uns nicht bestätigt werden. In Bezug auf die Häufigkeit der Hämatomentstehung ergab sich zwar kein signifikanter p-Wert, dieser lag aber mit einem Wert von 0,0545 nur knapp oberhalb der Signifikanzgrenze. Tendenziell neigten demnach eher die jüngeren Patienten zu einer Hämatomentstehung.

Zwei Studien aus den Jahren 1992 und 2004 konnten keine signifikante Korrelation zwischen dem Alter der Patienten und einer erhöhten Blutungsrate nach Biopsie nachgewiesen werden (Parrish 1992, Whittier und Korbet 2004 b).

\subsection{Die Nadeldicke als Risikofaktor für Komplikationen}

Als weiterer Einflussfaktor auf das Blutungsrisiko bei Nierenbiopsien wurde die Nadeldicke untersucht. Die meisten Nierenbiopsien in unserer Studie wurden mit einer 14G Nadel durchgeführt, bei einigen Patienten wurde eine 16G Nadel verwendet und nur in Einzelfällen eine 18G Nadel. Es konnte weder für die Hämatomentstehung oder den Hb-Abfall > $1 \mathrm{~g} / \mathrm{dl}$ innerhalb von 24 Stunden nach Biopsie, noch für die Komplikationsrate oder Komplikationsarten eine Signifikanz 
nachgewiesen werden. Die Nadeldicke hatte in unserer Studie demnach keinen Einfluss auf das Blutungsrisiko.

Bereits mehrere Studien in der Vergangenheit untersuchten die ideale Nadeldicke bei Nierenbiopsien. Mostbeck et al. untersuchten bereits 1989 den Unterschied zwischen 16G und 18G Nadeln bei perkutaner Nierenbiopsie. Sie kamen zu dem Ergebnis, dass die Komplikationsraten zwischen diesen beiden Nadelstärken sich nicht signifikant unterscheiden, mit der etwas dickeren 16G Nadel aber die Diagnosestellung anhand des gewonnenen Gewebes besser sei (Mostbeck et al. 1989).

Song und Cronan untersuchten den Unterschied zwischen einer 14G und einer 18G Nadel in Bezug auf die Erholungsrate des Gewebes und die Komplikationsrate. Die 14G Nadel unterschied sich bei beiden Parametern nicht signifikant von der 18G Nadel, es konnten aber mehr Glomeruli zur Beurteilung gewonnen werden (Song und Cronan 1998).

Nicholson et al. untersuchten in einer Studie an 100 Patienten mit einer Transplantatniere den Einfluss der Nadeldicke auf Komplikationen und den Erhalt des Gewebes (Nicholson et al. 2000). Dazu wurden die Patienten in Gruppen mit den Nadelgrößen 14G, 16G und 18G randomisiert. Als Resultat zeigte sich, dass die Gewebeproben mit der 14G Nadel am größten waren und sich die Proben mit den 14 und 16G Nadeln als diagnostisch wertvoller erwiesen als die Proben, die mit der kleinen 18G Nadel entnommen wurden. Bei der Handhabung während der Biopsie zeigte sich kein Unterschied in den drei Gruppen. Auch in der Komplikationsrate zeigte sich kein signifikanter Unterschied, allerdings klagten die Patienten, die mit der 14G Nadel biopsiert wurden über mehr Schmerzen als die Patienten aus den anderen beiden Gruppen. Alle drei Nadelgrößen waren also in der Sicherheit und Handhabung gleich, während die größeren Nadeln mehr Gewebe und Glomeruli gewannen und damit diagnostisch nützlicher waren, aber vor allem die größte 14G Nadel auch mit mehr Schmerzen verbunden war.

Kolb et al. veröffentlichten 1994 eine Studie, bei der 369 Nierenbiopsien an Transplantatnieren untersucht wurden. Die Biopsien wurden entweder mit einer 14G Franklin-Silverman-Nadel oder mit einer 18G automatischen Biopsienadel durchgeführt. Die Franklin-Silverman-Nadel hatte eine etwas höhere Komplikationsrate, konnte aber auch mehr Glomeruli erzielen als die automatische 18G Nadel. Letztlich war das Intervall zwischen Transplantation und 
Biopsie für die Komplikationsrate ausschlaggebender als die Art und Dicke der Biopsienadel (Kolb et al. 1994).

Anhand der Ergebnisse der angeführten Studien und auch der Ergebnisse aus unserer Studie lässt sich daraus folgern, dass eine 14G Nadel für die perkutane Nierenbiopsie sehr gut geeignet ist, da sich kein signifikanter Unterschied in der Komplikationsrate zu den anderen Nadelstärken zeigt. In mehreren Studien konnte mit der 14G Nadel mehr Gewebe gewonnen werden als zum Beispiel mit einer 18G Nadel (Kolb et al. 1994, Nicholson et al. 2000).

\subsection{Beobachtungszeitraum und Dauer des stationären Aufenthaltes}

Auch der Beobachtungszeitraum und damit auch die Dauer des stationären Aufenthaltes nach der Nierenbiopsie ist ein wichtiges Kriterium und sollte nicht zu kurz sein, um mögliche schwere Komplikationen nicht zu übersehen, aber auch nicht zu lange, um die Patienten möglichst kurzzeitig in stationärer Behandlung halten zu müssen.

In unserer Studie wurden die meisten Patienten einen Tag vor der Biopsie stationär aufgenommen und für 24 Stunden nach der Biopsie beobachtet, sodass die Patienten am Abend des Folgetages bei unkompliziertem Verlauf das Krankenhaus wieder verlassen konnten. Die Daten der Dauer des stationären Aufenthaltes in Bezug auf eventuelle Komplikationen waren nicht signifikant, sind aber auch nicht aussagekräftig, da viele Patienten nach der Nierenbiopsie unabhängig davon andere Komplikationen, zum Beispiel kardialer Genese entwickelten oder wegen anderer Krankheiten stationär aufgenommen wurden und erst im Laufe des stationären Aufenthaltes eine Nierenkrankheit entwickelten. Daher ist die Dauer des stationären Aufenthaltes in vielen Fällen wesentlich länger, als aufgrund der Nierenbiopsie erforderlich gewesen wäre. Trotzdem kamen wir anhand unserer Ergebnisse zu dem Schluss, dass bei normalem Verlauf der Nierenbiopsie eine Entlassung nach 24 Stunden angestrebt werden kann, wenn sich in diesem Zeitraum kein Anhalt für Komplikationen ergibt und keine anderen Krankheiten einen längeren Aufenthalt erforderlich machen. 
In mehreren Studien wurde bereits untersucht, welches die optimale Beobachtungsperiode nach perkutaner Nierenbiopsie ist.

Whittier und Korbet kamen in ihrer 2004 veröffentlichten Studie, in der sie den Zeitpunkt des Auftretens von Komplikationen und die Art der Komplikationen untersuchten, zu dem Urteil, dass ein Beobachtungszeitraum von 24 Stunden nach Biopsie als ideal anzusehen ist. Mit einem Überwachungszeitraum von 24 Stunden konnten 98\% aller Komplikationen und 100\% der schweren Komplikationen erfasst werden. Innerhalb der ersten 8 Stunden nach der Biopsie konnten lediglich $67 \%$ der Komplikationen erfasst werden und innerhalb der ersten 12 Stunden waren es 85\%. Dabei wurden jeweils auch schwere, behandlungsbedürftige Komplikationen nicht erfasst. Diese Zeiträume sind deshalb als zu kurz anzusehen (Whittier und Korbet 2004 b).

Marwah und Korbet bewiesen in einer weiteren Studie, dass ein Beobachtungszeitraum von 24 Stunden ideal ist, da bei einem Beobachtungszeitraum unter acht Stunden über 20\% der Komplikationen übersehen werden, während bei einem Zeitraum von 24 Stunden nur 2\% der Komplikationen nicht erfasst werden, wobei alle schweren interventionspflichtigen Komplikationen innerhalb dieses Zeitraumes auftraten (Marwah und Korbet1996). Allerdings gibt es auch Daten, die belegen, dass die perkutane Nierenbiopsie auch als ambulanter Eingriff durchgeführt werden kann.

Fraser und Fairley führten eine Studie durch, bei der bei 118 Patienten eine perkutane Nierenbiopsie als ambulanter Eingriff durchgeführt wurde und stellten diese 232 Patienten gegenüber, bei denen derselbe Eingriff stationär vollzogen wurde. Sie konnten keinen signifikanten Unterschied in der Komplikationsrate zwischen den beiden Gruppen nachweisen und kamen zu dem Schluss, dass die perkutane Nierenbiopsie als ambulanter Eingriff ein sicheres Verfahren darstellt (Fraser und Fairley 1995).

\subsection{Der Hb-Wert als Risikofaktor für Komplikationen}

Desweiteren bestimmten wir in unserer Studie den $\mathrm{Hb}$-Wert vor der Biopsie und untersuchten, ob sich ein Zusammenhang zur Hämatomentstehung, Hb-Abfall > 1 g/dl innerhalb von 24 Stunden nach Biopsie, Komplikation oder den einzelnen Komplikationsarten ergab. 
Es sollten nur Patienten mit einem Ausgangs- $\mathrm{Hb}>10 \mathrm{~g} / \mathrm{dl}$ biopsiert werden, in einigen Fällen wurde allerdings auch von dieser Regel abgewichen und eine Nierenbiopsie bei niedrigeren Werten durchgeführt.

In unserer Studie ergab sich ein signifikantes Ergebnis in Bezug auf den Hb-Wert für die Komplikationsrate und bei der Aufschlüsselung nach den Komplikationsarten für den klinisch relevanten $\mathrm{Hb}$-Abfall. Dieses Ergebnis ist sehr gut nachzuvollziehen, da Patienten mit einem niedrigeren präbiopsären $\mathrm{Hb}$-Wert eher die Bereitstellung oder Gabe einer Blutkonserve benötigten, da sie schneller einen kritisch niedrigen Bereich ihres $\mathrm{Hb}$-Wertes erreichten als die Patienten mit einem höheren präbiopsären $\mathrm{Hb}$-Wert. Allerdings konnte auch für die Komplikationsart Blutung eine Tendenz zu höherer Blutungsrate bei niedrigem präbiopsärem Hb-Wert nachgewiesen werden ( $p$-Wert 0,0999). Dieses Ergebnis war zwar nicht signifikant, lässt aber vermuten, dass Patienten mit niedrigem $\mathrm{Hb}$ Wert ein höheres Risiko für Blutungen nach Biopsie aufweisen als Patienten mit einem höheren $\mathrm{Hb}$-Wert. Allerdings reicht diese Tendenz nicht aus, um zukünftig anhand des $\mathrm{Hb}$-Wertes das Risiko für Blutungen nach Biopsie besser prognostizieren zu können.

Marwah und Korbet konnten in ihrer Studie aus dem Jahre 1996 einen signifikant niedrigeren $\mathrm{Hb}$-Wert bei Patienten mit Komplikationen nach Biopsie nachweisen im Vergleich zu den Patienten ohne Komplikation (Marwah und Korbet 1996).

\subsection{Die Blutgerinnung als Risikofaktor für Komplikationen}

Auch die Blutgerinnung kann eine Rolle spielen in Bezug auf die Blutungskomplikationen nach perkutaner Nierenbiopsie. Aus diesem Grunde wurde in unserer Studie vor der Biopsie ein Gerinnungslabor gemacht, das die PTT und den Quickwert umfasste. Für beide Werte konnte in den untersuchten Bereichen Hämatomentstehung, Hb-Abfall $>1 \mathrm{~g} / \mathrm{dl}$ innerhalb von 24 Stunden nach Biopsie, Komplikation und Komplikationsart keine Signifikanz nachgewiesen werden. Anhand unserer Ergebnisse erscheinen beide Werte nicht geeignet, um ein erhöhtes Risiko für Blutungskomplikationen nachweisen zu können. Allerdings zeigte sich bei dem Quickwert mit nominal skalierten Daten eine Tendenz im Zusammenhang mit der Hämatomentstehung ( $p$-Wert 0,0639). 47,37\% der 
Patienten mit einer Hämatomentwicklung hatten einen Quickwert $>120 \%$, während nur 28,85\% der Patienten ohne Hämatom einen Quickwert $>120 \%$ aufwiesen. Da diese Tendenz eines pathologischen Quickwertes mit höherer Hämatomrate durch die ordinal skalierten Daten nicht bestätigt werden konnte ( $p$ Wert 0,3198), kann aber auch hier kein wirklicher Einfluss auf ein erhöhtes Blutungsrisiko nach Biopsie nachgewiesen werden.

Auch für die PTT ließ sich eine erhöhte Tendenz nachweisen. Die Auswirkung der PTT auf die Entstehung einer Makrohämaturie nach Biopsie ergab einen $p$-Wert von 0,0620. Patienten mit einer kürzeren PTT haben tendenziell häufiger eine Makrohämaturie entwickelt als Patienten mit einer normalen PTT. Da die Makrohämaturie in allen Fällen spontan sistierte und zu den weniger ernsthaften Komplikationen ohne klinische Relevanz gehörte und zudem keine Signifikanz nachgewiesen werden konnte, ergibt sich aus diesem Ergebnis keine klinische Konsequenz.

Auch Davis und Chandler fanden in ihrer 1995 veröffentlichten Studie keinen Anhalt auf eine Korrelation zwischen Quick oder PTT und erhöhtem Blutungsrisiko (Davis und Chandler 1995).

Manno et al. konnten jedoch in ihrer Studie aus dem Jahre 2004 zeigen, dass Patienten mit einer erhöhten PTT ein signifikant höheres Blutungsrisiko aufweisen als Patienten mit einer normalen PTT (Manno et al. 2004). Trotzdem ist die Datenlage nicht ausreichend um auf einen Zusammenhang zwischen einer erhöhten PTT und einem erhöhten Blutungsrisiko zu schließen.

\subsection{Die Nierenfunktion als Risikofaktor für Komplikationen}

Da die Nierenfunktion bereits in mehreren Studien als Risikofaktor für die Entstehung von Blutungskomplikationen nach der perkutanen Nierenbiopsie untersucht wurde, erhoben wir als Parameter der Nierenfunktion den Kreatininwert, die Kreatininclearance und die GFR anhand der MDRD-Formel. Die MDRD-Formel berechnet die GFR zwar nur näherungsweise, ist aber trotzdem oftmals aussagekräftiger als die Kreatininclearance, da Fehler, wie zum Beispiel beim Sammeln des Urins, vermieden werden. 
Für die Hämatomentstehung und einen Hb-Abfall $>1 \mathrm{~g} / \mathrm{dl}$ innerhalb der ersten 24 Stunden nach Biopsie konnte für keinen der drei Werte eine Signifikanz nachgewiesen werden. Allerdings zeigte sich der Serumkreatininwert hoch signifikant für die Komplikationsrate $(p-W e r t<0,0001)$. Ein erhöhtes Kreatinin war auch für alle drei Komplikationsarten signifikant, und für die Blutungskomplikationen hierbei ebenfalls hoch signifikant ( $p$-Wert 0,0001 für Blutung, 0,0021 für Makrohämaturie, 0,0040 für klinisch relevanten $\mathrm{Hb}$-Abfall).

Die Kreatininclearance war für keine der untersuchten Komplikationen signifikant, wobei sich allerdings bei den Blutungskomplikationen eine Tendenz von eingeschränkter Kreatininclearance zu erhöhter Blutungskomplikationsrate und auch allgemeiner Komplikationsrate nachweisen ließ ( $p$-Wert 0,0823 für Blutung und 0,0986 für Komplikationsrate). Untermauert wird dieses Ergebnis durch den MDRD-Wert, bei dem sich nach ordinalskalierten Daten eine Tendenz zu einer höheren Blutungskomplikationsrate zeigte ( $p$-Wert 0,0763$)$. Dies konnte allerdings durch die nominal skalierten Daten nicht bestätigt werden ( $p$-Wert 0,2424). Für die Komplikationsrate zeigte sich in Bezug auf den MDRD-Wert eine Signifikanz sowohl bei den ordinal, als auch bei den nominal skalierten Daten ( $p$-Wert 0,0092 ordinal und 0,0338 nominal). $80 \%$ der Patienten mit einer Komplikation hatten eine nach der MDRD-Formel berechnete GFR von $<30 \mathrm{ml} / \mathrm{min} / 1,73 \mathrm{~m}^{2} \mathrm{KO}$, während dies nur bei $36,62 \%$ der Patienten ohne Komplikation der Fall war. Dagegen hatten nur 20\% der Patienten mit Komplikation eine GFR > $30 \mathrm{ml} / \mathrm{min} / 1,73 \mathrm{~m}^{2} \mathrm{KO}$, während in der Gruppe ohne Komplikation $63,38 \%$ der Patienten eine solche GFR aufwiesen.

Ein signifikantes Ergebnis zeigte sich auch bei der Untersuchung des Einflusses der MDRD-Formel auf die Komplikationsart Makrohämaturie mit ordinal skalierten Daten. Dieses Ergebnis konnte mit der Berechnung anhand nominal skalierter Daten nicht bestätigt werden.

Eine eingeschränkte GFR gemessen als Kreatininclearance oder besser durch die MDRD-Formel bestimmt, hat sich in unserer Studie also als Risikofaktor für Blutungskomplikationen nach perkutaner Nierenbiopsie gezeigt. Es kann allerdings anhand unserer Ergebnisse kein Rückschluss auf die Art der Komplikation gemacht werden. Trotzdem ist nicht auszuschließen, dass eine eingeschränkte Nierenfunktion auch auf bestimmte Komplikationsarten größeren Einfluss nimmt als auf andere, da die Stichprobenumfänge in Aufteilung nach den 
Komplikationsarten teilweise sehr unterschiedlich groß waren und so falsch negative Ergebnisse nicht ausgeschlossen werden können.

Auch ein erhöhter Kreatininwert als Resultat einer eingeschränkten Nierenfunktion zeigte sich in unserer Studie als Hinweis auf ein erhöhtes Blutungsrisiko nach perkutaner Nierenbiopsie.

Ein ähnliches Ergebnis zeigte sich bereits in mehreren Studien. Whittier und Korbet konnten in inrer Studie zeigen, dass schwere Komplikationen bei Patienten mit einem Serumkreatinin über $5 \mathrm{mg} / \mathrm{dl}$ zweimal häufiger auftraten als bei Patienten mit normalem Serumkreatinin (Whittier und Korbet 2004 b).

Zu einem ähnlichen Ergebnis kamen Shidham et al., die nachweisen konnten, dass Patienten mit einem erhöhten Serumkreatinin ein signifikant höheres Risiko für Blutungskomplikationen nach Nierenbiopsien haben als Patienten mit einem niedrigeren Serumkreatininspiegel. Zudem kamen sie zu dem Ergebnis, dass Patienten mit einem Serumkreatininspiegel $>2 \mathrm{mg} / \mathrm{dl}$ ein 5,89-fach erhöhtes Risiko für Blutungskomplikationen nach Nierenbiopsie haben als Patienten mit einem Serumkreatininspiegel $<2 \mathrm{mg} / \mathrm{dl}$ (Shidham et al. 2005).

Allerdings gibt es auch Studien, die zeigen, dass ein niedriges Serumkreatinin mit einem erhöhten Risiko für Blutungskomplikationen einhergeht, wobei diese Daten bei logistischer Regression nicht bestätigt werden konnten (Stiles et al. 2001). Marwah und Korbet hingegen konnten keinen Zusammenhang zwischen dem Kreatininspiegel und der Komplikationsrate nachweisen (Marwah und Korbet 1996).

Da die Datenlage in Bezug auf die Nierenfunktion und den Kreatininspiegel im Zusammenhang mit Blutungskomplikationen bei perkutaner Nierenbiopsie immer noch sehr widersprüchlich ist, ist auch dieser Parameter nicht dazu geeignet ein erhöhtes Risiko sicher zu diagnostizieren.

Trotzdem zeigten sich in unserer Studie sehr deutlich Hinweise darauf, dass eine eingeschränkte Nierenfunktion, vor allem im Sinne eines erhöhten Kreatinins und einer eingeschränkten GFR ein wichtiger Risikofaktor in Hinsicht auf die Blutungskomplikationen ist.

Auch die Unterscheidung zwischen nierentransplantierten Patienten und Patienten mit Eigenniere mit einer jeweils gesonderten Berechnung in Bezug auf die Komplikationsrate ergab den Kreatininwert als bedeutenden Risikofaktor für Komplikationen. Die Bedeutung der GFR zeigte sich weniger deutlich und kann 
auch nicht als prognostischer Faktor für das individuelle Komplikationsrisiko verwendet werden.

\subsection{Begleiterkrankungen als Risikofaktoren für Komplikationen}

In unserer Studie untersuchten wir auch den Einfluss von bestimmten Begleiterkrankungen auf das Blutungsrisiko nach perkutaner Nierenbiopsie. Untersucht wurde der Zusammenhang zwischen arterieller Hypertonie oder Diabetes mellitus oder Patienten mit einer Transplantatniere und dem Auftreten von Hämatomen nach Biopsie, einem Hb-Abfall > $1 \mathrm{~g} / \mathrm{dl}$ innerhalb von 24 Stunden nach Biopsie, Komplikationen und der drei Komplikationsarten Makrohämaturie, Blutung und klinisch relevantem $\mathrm{Hb}$-Abfall.

Für keine dieser Begleiterkrankungen konnte eine Signifikanz für die angeführten Parameter nachgewiesen werden. Wir konnten somit nicht nachweisen, dass Patienten mit den Begleiterkrankungen arterielle Hypertonie oder Diabetes mellitus oder auch Patienten mit Transplantatniere ein erhöhtes Risiko für Blutungskomplikationen nach perkutaner Nierenbiopsie aufweisen.

Shidham et al. untersuchten in einer Studie mit 645 perkutanen Nierenbiopsien den Einfluss von arterieller Hypertonie auf die Blutungsrate. Sie konnten in ihrer Studie zeigen, dass ein erhöhter Blutdruck mit einer erhöhten Blutungsrate einhergeht. Dabei hatten Patienten mit einer bekannten arteriellen Hypertonie ein 3,74-fach erhöhtes Risiko gegenüber Patienten ohne arterielle Hypertonie. Das Risiko war erhöht bei erhöhtem systolischem Blutdruck > $160 \mathrm{mmHg}$, diastolischem Blutdruck $>100 \mathrm{mmHg}$ und mittlerem arteriellem Blutdruck $>120$ $\mathrm{mmHg}$ (Shidham et al. 2005).

Auch Eiro et al. (2005) fanden in ihrer Studie mit 359 Patienten einen Zusammenhang zwischen arterieller Hypertonie und der Blutungsrate bei perkutaner Nierenbiopsie und kamen zu dem Schluss, dass Patienten mit einer arteriellen Hypertonie nach perkutaner Nierenbiopsie genauer beobachtet werden müssen als Patienten ohne Begleiterkrankungen.

Als prognostischer Faktor für Blutungskomplikationen kann eine vorbestehende arterielle Hypertonie jedoch nicht herangezogen werden, da die Ergebnisse 
diverser Studien zu uneinheitlich sind und wohl auch die individuelle Variabilität eine große Rolle spielt.

Die Patienten mit einer Transplantatniere hatten in unserer Studie kein erhöhtes Risiko für Komplikationen nach Nierenbiopsie. Kein Patient verlor die transplantierte Niere aufgrund von Komplikationen bei oder nach der Biopsie.

Lange Zeit galt es als kontraindiziert, eine transplantierte Niere zu biopsieren. Zahlreiche Studien wurden durchgeführt, um die Komplikationsrate besser abschätzen zu können.

Eine Studie mit mehr als 200 Biopsien konnte zeigen, dass die perkutane Nierenbiopsie unter Ultraschallkontrolle und mit einem automatisierten Biopsiegerät ein sehr sicheres und präzises Verfahren darstellt und das Risiko für Komplikationen signifikant mindert gegenüber dem Biopsieverfahren mit einer Tru-Cut-Nadel (Mahoney et al. 1993).

Wilczek untersuchte 1129 Biopsien and 513 Patienten mit einer transplantierten Niere und kam zu dem Ergebnis, dass eine perkutane Nierenbiopsie an einer Transplantatniere ein gewisses Risiko für Komplikationen bis hin zu dem Verlust der Transplantatniere birgt, dass aber der Nutzen der durch die Nierenbiopsie gewonnenen Erkenntnisse höher ist als das Komplikationsrisiko. Daraus folgerte er, dass die perkutane Nierenbiopsie an Transplantatnieren auch weiterhin ein wichtiges diagnostisches Mittel bei Komplikationen nach Nierentransplantation darstellt (Wilczek 1991).

Beckingham et al. untersuchten 210 nierentransplantierte Patienten mit einer Nierenbiopsie. Sie kamen zu dem Ergebnis, dass selbst unter sorgfältiger Ultraschallkontrolle eine wichtige Komplikationsrate bestehen bleibt. Da das Risiko für Blutungen nach Biopsie signifikant höher war, wenn medulläre Nierenanteile gewonnen wurden, schlossen sie daraus, dass die perkutane Nierenbiopsie bei Transplantatnieren möglichst oberflächlich erfolgen sollte (Beckingham et al. 1994). 


\subsection{Die nephrologische Diagnose als Risikofaktor für Komplikationen}

Als letzter Einflussparameter wurde in unserer Studie die nephrologische Diagnose, die anhand des Biopsates gestellt wurde, evaluiert. Es wurde der Einfluss auf Hämatomentstehung, Hb-Abfall $>1 \mathrm{~g} / \mathrm{dl}$ innerhalb von 24 Stunden nach Biopsie, Komplikationsrate und Komplikationsarten untersucht.

Als nephrologische Diagnose galten Nephrosklerose, FSGS, IgA-Nephritis, Lupusnephritis, membranoproliferative Nephritis, membranöse GN, Transplantat - Glomerulopathie, minimal change GN, diabetische Nephropathie und andere (bestehend aus: kein Anhalt für GN, Cholesterin-Embolie, Tubulus-Atrophie, akutes Nierenversagen, Amyloidose, Komplementfaktor-C3-positive GN, chronisch interstitieller Schaden, kein Parenchym).

Es konnte kein signifikanter Zusammenhang zwischen der nephrologischen Diagnose und den angeführten Parametern nachgewiesen werden. Stratta et al. veröffentlichten 2007 eine Studie, in der sie 1387 Patienten untersuchten, die innerhalb von 30 Jahren nierenbiopsiert wurden. In ihrer Studie konnten sie nachweisen, dass eine Nierennekrose im Endstadium mit signifikant erhöhtem Risiko für Komplikationen einhergeht (Stratta et al. 2007).

Die nephrologische Diagnose kann aber trotzdem nicht als prognostischer Faktor für das Auftreten von Blutungskomplikationen nach perkutaner Nierenbiopsie dienen, da bei vielen Krankheitsbildern eine perkutane Nierenbiopsie zur Diagnosestellung absolut unerlässlich ist. Zudem konnte noch kein ausreichender Beweis für die Abhängigkeit des Blutungsrisikos von der nephrologischen Diagnose gestellt werden. 


\section{Zusammenfassung}

Blutungskomplikationen sind das Hauptproblem bei perkutanen Nierenbiopsien. Sie können sich als Makrohämaturie, als perirenale Blutung und als Hb-Abfall äußern. Das Risiko für Blutungen nach Biopsie ist immer noch sehr schwierig zu prognostizieren. Viele Parameter wurden diesbezüglich in diversen Studien eruiert, bisher aber ohne Erfolg.

In dieser Untersuchung wurden retrospektiv die Daten von 100 Patienten erhoben, bei denen in den Jahren 2004 bis 2007 an der Universitätsmedizin Göttingen eine perkutane Nierenbiopsie durchgeführt wurde. Ziel der Untersuchung war, die Auswirkung des platelet function analyzers PFA- $100^{\mathrm{TM}}$ auf das Blutungsrisiko nach Biopsie zu erheben. Zudem wurde der Einfluss zahlreicher anderer Faktoren auf das Blutungsrisiko eruiert.

Für den PFA-100 ${ }^{\mathrm{TM}}$-Wert konnte keine Auswirkung auf das Blutungsrisiko nachgewiesen werden. PFA-100 ${ }^{\mathrm{TM}}$ zeigte für keinen der erhobenen Parameter Signifikanz. Eine verlängerte Verschlusszeit des PFA-100 ${ }^{\mathrm{TM}}$-Wertes korrelierte demnach nicht mit dem Blutungsrisiko.

Einen signifikanten Einfluss auf Blutungskomplikationen zeigte in der Untersuchung lediglich der Kreatininwert in Bezug auf die Komplikationsrate und alle Komplikationsarten. Patienten mit einem hohen Kreatininspiegel vor der Biopsie sollten deshalb danach über 24 Stunden engmaschig kontrolliert und besonders intensiv überwacht werden. Auch sollten diese Patienten auf das erhöhte Blutungsrisiko hingewiesen werden. Es konnte zudem eine Signifikanz für die MDRD-Formel in Bezug auf die allgemeine Komplikationsrate und bedingt auch auf die Makrohämaturie nachgewiesen werden.

Zudem zeigte sich ein erniedrigter $\mathrm{Hb}$-Wert als signifikanter Risikofaktor für einen klinisch relevanten $\mathrm{Hb}$-Abfall. Dies ist allerdings nicht von Konsequenz für die Prognostizierung eines individuellen Blutungsrisikos, da Patienten mit einem niedrigen $\mathrm{Hb}$-Wert vor Biopsie schneller Erythrozytenkonzentrate benötigen als Patienten mit einem normalen $\mathrm{Hb}$-Wert. Der Hb-Wert ist demnach nicht zur Prognostizierung eines Blutungsrisikos oder $\mathrm{Hb}$-Abfalls nach Biopsie geeignet.

Diese Studie konnte somit keinen zuverlässigeren Parameter als die Einschränkung der Nierenfunktion zur besseren Vorhersagbarkeit des individuellen Risikos für Komplikationen nach perkutaner Nierenbiopsie nachweisen. 


\section{Anhang}

\subsection{Abkürzungsverzeichnis}

\begin{tabular}{|c|c|}
\hline Abb & Abbildung \\
\hline ADP & Adenosin-5'-Diphosphat \\
\hline ANCA & Antineutrophile zytoplasmatische Antikörper \\
\hline CBA & collagen binding activity assay \\
\hline DDAVP & Deamino-8-D-Arginin-Vasopressin \\
\hline $\mathrm{dl}$ & Deziliter \\
\hline EPI & Epinephrin \\
\hline FSGS & fokal segmentale Glomerulosklerose \\
\hline$g$ & Gramm \\
\hline G & Gauge \\
\hline GFR & glomeruläre Filtrationsrate \\
\hline GN & Glomerulonephritis \\
\hline $\mathrm{h}$ & Stunde \\
\hline $\mathrm{Hb}$ & Hämoglobin \\
\hline $\lg A$ & Immunglobulin A \\
\hline $\mathrm{KO}$ & Körperoberfläche \\
\hline $\mathrm{m}^{2}$ & Quadratmeter \\
\hline MDRD & Modification of Diet in Renal Disease \\
\hline $\mathrm{mg}$ & Milligramm \\
\hline$\mu \mathrm{g}$ & Mikrogramm \\
\hline $\min$ & Minute \\
\hline $\mathrm{ml}$ & Milliliter \\
\hline$\mu \mathrm{m}$ & Mikrometer \\
\hline MW & Mittelwert \\
\hline $\mathrm{N}$ & Stichprobenumfang \\
\hline PFA & platelet function analyzer \\
\hline PTCA & perkutane transluminale koronare Angioplastie \\
\hline PTT & partielle Thromboplastinzeit \\
\hline sec & Sekunde \\
\hline SF & Standardfehler \\
\hline
\end{tabular}


sog

Tab

TEG

V

VWD

VWF sogenannte

Tabelle

Thromboelastograph

Vena

von-Willebrand-Krankheit

von-Willebrand-Faktor 


\subsection{Abbildungsverzeichnis}

Abb.1: Übersicht über die absoluten und relativen Indikationen zur Nierenbiopsie (Seite 1)

Abb.2: Materialien zur Durchführung einer Nierenbiopsie; Quelle: Begleit - CD zum Modul Nephrologie am Universitätsklinikum Göttingen, Abteilung Nephrologie und Rheumatologie Georg - August Universität Göttingen 2007 (Seite 9)

Abb. 3: Durchführung einer perkutanen Nierenbiopsie unter Ultraschallüberwachung; Quelle: Begleit - CD zum Modul Nephrologie am Universitätsklinikum Göttingen, Abteilung Nephrologie und Rheumatologie Georg - August Universität Göttingen 2007 (Seite 10)

Abb. 4: Ultraschallgesteuerte Punktion der Niere. Die gelben Striche zeigen den Stichkanal; Quelle: Begleit - CD zum Modul Nephrologie am Universitätsklinikum Göttingen, Abteilung Nephrologie und Rheumatologie Georg - August Universität Göttingen 2007 (Seite 11)

Abb. 5: Übersicht über die Häufigkeit von Hämatomen, Hb-Abfall $>1 \mathrm{~g} / \mathrm{dl}$, symptomatische Komplikation (Seite 22) 


\section{Literaturverzeichnis}

Abbott KC, Musio FM, Chung EM, Lomis NL, Lane JD, Yuan CM (2002):

Transjugular renal biopsy in high-risk patients: an American case series.

BMC Nephrology $\underline{3}, 5-11$

Beckingham IJ, Nicholson ML, Bell PRF (1994): Analysis of factors associated

with complications following renal transplant needle core biopsy. Br J Urol

$\underline{73}, 13-15$

Blanco JJA, Villar BB, Juzgado A, Martinez RM (2006): Assessment of the PFA-

100 system for the measurement of bleeding time in oral surgery. Med Oral Patol Oral Cir Bucal 11, 514-519

Borzini P, Lazzaro A, Mazzucco L (1999): Evaluation of the hemostatic function of stored platelet concentrates using the platelet function analyzer (PFA-100). Haematologica 84, 1104-1109

Burton DR: Indications for and complications of renal biopsy. In: UpToDate, Basow DS (Ed), UpToDate, Waltham, MA 2004

Chen YP, Yu YP, Huang HE (2000): Complications of percutaneous renal biopsy: an analysis of 1000 consecutive biopsies. Zhongua Nei Ke Za Zhi $\underline{32}$, Abstract

Cluzel P., Martinez F, Bellin MF, Michalik Y, Beaufils H, Jouanneau C, Lucidarme O, Deray G, Grenier PA (2000): Transjugular versus percutaneous renal biopsy for the diagnosis of parenchymal disease: comparison of sampling effectiveness and complications. Radiology $\underline{215}, 689-693$

Conger KB, Sarembock LA (1961): Open renal needle biopsy under local anaesthesia. Ann Surg 154, 345-350

Davis CL, Chandler WL (1995): Thromboelastograpgy for the predicition of bleeding after transplant renal biopsy. J Am Soc Nephrol $\underline{6}, 1250-1255$

Eiro M, Katoh T, Watanabe T (2005): Risk factors for bleeding complications in percutaneous renal biopsy. Clin Exp Nephrol $\underline{9}$, 40-45

Fattobrutto M, Pradier O, Schmartz D, Ickx B, Barvais L (2003): Does the platelet function analyzer (PFA - 100) predict blood loss after cardiopulmonary bypass? Br J Anaesth $\underline{90}, 692-693$ 
Favaloro EJ (2001): Utility of the PFA-100 for assessing bleeding disorders and monitoring therapy: a review of analytical variables, benefits and limitations. Haemophilia $\underline{7}, 170-179$

Favaloro EJ, FaceyD, Henniker A (1999): Use of a novel platelet function analyzer (PFA-100) with high sensitivity to disturbances in von Willebrand factor to screen for von Willebrand's Disease and other disorders. Am J Hematol 62, $165-174$

Favaloro EJ, Kershaw G, Bukuya M, Hertzberg M, Koutts J (2001): Laboratory diagnosis of von Willebrand disorder (VWD) and monitoring of DDAVP therapy: efficacy of the PFA - 100 and vWF: CBA as combined diagnostic strategies. Haemophilia $\underline{7}, 180-189$

Feneberga R, Schaefera F, Ziegerb B, Waldherrc R, Mehlsa O, Schärera K (1998): Percutaneous renal biposy in children: A 27 - year experience. Nephron $\underline{79}, 438-446$

Fine DM, Arepally A, Hofmann LV, Mankowitz SG, Atta MG (2004): Diagnostic utility and safety of transjugular kidney in the obese patient. Nephrol Dial Transplant $\underline{19}$, 1798-1802

Forestier F, Coiffic A, Mouton C, Ekouevi D, Chêne G, Janvier G (2002): Platelet function point - of - care tests in post - bypass cardiac surgery: are they relevant? Br J Anaesth $\underline{89}, 715-721$

Fraser IR, Fairley KF (1995): Renal biopsy as an outpatient procedure. Am J Kidney Dis $\underline{25}$, 876-878

Fressinaud E, Veyradier A, Truchaud F, Martin I, Boyer - Neumann C, Trossaert M, Meyer D (1998): Screening for von Willebrand Disease with a new analyzer using high shear stress: A study of 60 cases. Blood $\underline{91}, 1325-$ 1331

Gimenez LF, Micali S, Chen RN, Moore RG, Kavoussi LR, Scheel PJ (1998): Laparoscopic renal biopsy. Kidney Int $\underline{54}, 525-529$

González-Michaca L, Chew-Wong A, Soltero L, Gamba G, Correa-Rotter R (2000): Percutenous kidney biopsy, analysis of 26 years: complication rate and risk factors. Rev Invest Clin $\underline{52}, 125-131$

Gupta M, Haluck RS, Yang HC, Holman MJ, Ahsan N (2000): Laprasocopic assisted renal biopsy : An alternative to open approach. Am J Kidney Dis 36, 636-639 
Gwyn NB (1923): Biopsies and the completion of certain surgical procedures. Can Med Assoc J 13, 820-823, 1923

Haas M (1997): A reevaluation of routine electron microscopy in the examination of native renal biopsies. J Am Soc Nephrol $\underline{8}, 70-76$

Harrison P, Robinson MSC, Mackie IJ, McDonald SJ, Liesner R, Savidge GF, Pasi J, Machin SJ ( 1999): Performance of the platelet function analyser PFA - 100 in testing abnormalities of primary hemostasis. Blood Coagul Fibrinolysis $\underline{10}, 25-31$

Hayward CPM, Harrison P, Cattaneo M, Ortel TL, Rao AK (2006): Platelet function analyzer (PFA)-100 closure time in the evaluation of platelet disorders and platelet function. J Thromb Haemost 4 4 , 312-319

Hergesell O, Felten H, Andrassy K, Kühn K, Ritz E (1998): Safety of ultrasoundguided percutaneous renal biopsy - retrospective analysis of 1090 consecutive cases. Nephrol Dial Transplant 13, 975-977

Hézard N, Metz D, Nazeyrollas P, Droulle C, Elaerts J, Potron G, Nguyen P (2000): Use of the PFA-100 apparatus to assess platelet function in patients undergoing PTCA during and after infusion of cE3 Fab in the presence of other antiplatelet agents. Thromb Haemost $\underline{83}, 540-544$ Homoncik M, Jilma B, Hergovich N, Stohlawetz P, Panzer S, Speiser W (2000): Monitoring of Aspirin (ASA) Pharmacodynamics with the Platelet Function Analyzer PFA - 100. Thromb Haemost 83, 316-321

Jörstad S, Borander U, Berg KJ, Wideröe TE (1984): Evaluation of complications due to percutaneous renal biopsy: a clinical and angiographic study. Am J Kidney Dis $\underline{4}, 162-165$

Jouet P, Meyrier A, Mal F, Callard P, Guettier C, Stordeur D, Trinchet JC, Beaugrand M (1996): Transjugular renal biopsy in the treatment of patients with cirrhosis and renal abnormalities. Hepatology 24, 1143-1147

Khajehdehi P, Junaid SM, Salinas - Madrigal L, Schmitz PG, Bastani B (1999): Percutaneous renal biopsy in the 1990s: safety, value, and implications for early hospital discharge. Am J Kidney Dis $\underline{34}$, 92-97

Kolb LG, Velosa JA, Bergstralh EJ, Offord KP (1994): Percutaneous renal allograft biopsy. Transplantation $\underline{57}, 1742-1746$

Kottke-Marchant K, Powers JB, Brooks L, Kundu S, Christie DJ (1999): The effect of antiplatelet drugs, heparin and preanalytical variables on platelet 
function detected by Platelet Function Analyzer (PFA - 100). Clin Appl Thrombosis/Hemostasis $\underline{5}, 122-130$

Lind SE (1991): The bleeding time does not predict surgical bleeding. Blood $\underline{77}$, 2547-2552

Madaio MP(1990): Renal biopsy. Kidney Int 38, 529-542

Madan M, Berkowitz SD, Christie DJ, Jennings LK, Smit AC, Sigmon KN, Glazer

S, Tcheng JE (2001): Rapid assessment of glycoprotein Ilb/llla blockade with the platelet function analyzer (PFA-100) during percutaneous coronary intervention. Am Heart J 141, 226-233

Mahoney MC, Racadio JM, Merhar GL, First MR (1993): Safety and efficacy of kidney transplant biopsy: Tru-Cut needle vs sonographically guided biopty gun. AJR $\underline{160}$, 325-326

Mal F, Meyrier A, Callard P, Kleinknecht D, Altmann JJ, Beaugrand M (1992): The diagnostic yield of transjugular renal biopsy. Experience in 200 cases.

Kidney Int $\underline{41}, 445-449$

Manno C, Strippoli GFM, Arnesano L, Bonifati C, Campobasso N, Gesualdo L, Schena FP (2004): Predictors of bleeding complications in percutaneous unltrasound - guided renal biopsy. Kidney Int $\underline{66}, 1570-1577$

Marshall PW, Williams AJ, Dixon RM, Growcott JW, Warburton S, Armstrong J, Moores J (1997): A comparison of the effects of aspirin on bleeding time measured using the Simplate method and closure time measured using the PFA - 100, in healthy volunteers. Br J Clin Pharmacol 44, 151-155

Marwah DA, Korbet SM (1996): Timing of complications in percutaneous renal biopsy: what is the optimal period of observation? Am J Kidney Dis $\underline{28}$, 4752

Mattix H, Singh AK (1999): Is the bleeding time predictive prior to a percutaneous renal biopsy? Curr Opin Nephrol Hypertens $\underline{8}$, 715-718

Mendelssohn DC, Cole EH (1995): Outcomes of percutaneous kidney biopsy, including those of native solitary kidneys. Am J Kidney Dis $\underline{26}, 580-585$

Meola M, Barsotti G, Cupisti A, Buoncristiani E, Giovanetti S (1994): Free - hand ultrasound - guided renal biopsy: Report of 650 consecutive cases. Nephron $\underline{67}$, 425-430 
Metzler MA: Studie zur Bedeutung der In-vitro-Blutungszeitmessung mit dem PFA-100 für die Vorhersage peri- und postoperativer

Blutungskomplikationen in der Gynäkologie. Med. Diss. Aachen 2001

Meyrier A (2005): Transjugular renal biopsy. Update on hepato-renal needlework. Nephrol Dial Transplant 20, 1299-1302

Mimidis K, Papadopoulos V, Kartasis Z, Baka M, Tsatlidis V, Bourikas G, Kartalis $G$ (2004): Assessment of platelet adhesiveness and aggregation in mild acute pancreatitis using the PFA -100 system. J Pancreas $\underline{5}, 132-137$ Mostbeck GH, Wittich GR, Derfler K, Ulrich W, Walter RM, Herold C, Haller J, Tscholakoff D (1989): Opitmal needle size for renal biopsy: in vitro and in vivo evaluation. Radiology $\underline{173}$, 819-822

Nicholson ML, Wheatley TJ, Doughman TM, White SA, Morgan JDT, Veitch S, Furness PN (2000): A prospective randomized trial of three different sizes of core-cutting needle for renal transplant biopsy. Kidney Int $\underline{58}, 390-395$ Nyman RS, Cappelen - Smith J, al Suhaibani H, Alfurayh O, Shakweer W, Akhtar $M$ (1997): Yield and complications in percutaneous renal biopsy. A comparison between ultrasound - guided gun - biopsy and manual techniques in native and transplant kidneys. Acta Radiol $\underline{38}, 431-436$

Ortel TL, James AH, Thames EH, Moore KD, Greenberg CS (2000): Assessment of primary hemostasis by PFA - 100 analysis in a tertiary care center. Thromb Haemost 84, 93-97

Parrish AE (1992): Complications of percutaneous renal biopsy: a review of 37 years' experience. Clin Nephrol $\underline{38}, 135-141$

Podda GM, Bucciarelli P, Lussana F, Lecchi A, Cattaneo M (2007): Usefulness of PFA-100 testing in the diagnostic screening of patients with suspected abnormalities of hemostasis: comparison with the bleeding time. J Thromb Haemost $\underline{5}, 2393-2398$

Prakash J, Singh M, Tripathi K, Rai US (1994): Complications of percutaneous renal biopsy. J Indian Med Assoc 92, 395-396

Preda A, Van Dijk LC, Van Oostaijen JA, Pattynama PMT (2003): Complication rate and diagnostic yield of 515 consecutive ultrasound - guided biopsies of renal allografts and native kidneys using a 14-gauge Biopty gun. Eur Radiol $\underline{13}, 527-530$ 
Quiroga T, Goycoolea M, Munoz B, Morales M, Aranda E, Panes O, Pereira J, Mezzano D (2004): Template bleeding time and PFA-100 have low sensitivity to screen patients with hereditary mucocutaneous hemorrhages: comparative study in 148 patients. J Thromb Haemost 2, 892-898

Richards NT, Darby S, Howie AJ, Adu D, Michael J (1994): Knowledge of renal histology alters patient management in over $40 \%$ of cases. Nephrol Dial Transplant $\underline{9}, 1255-1259$

Rychlík I, Petrtýl J, Tesar V, Stejskalová A, Zabka J, Bruha R (2001): Transjugular renal biopsy - Our experience with 67 cases. Kidney Blood Press Res $\underline{24}$, 207-212

Shidham GB, Siddiqi N, Beres JA, Logan B, Nagaraja HN, Shidham SG, Piering WF (2005): Clinical risk factors associated with bleeding after native kidney biopsy. Nephrology 10, 305-310

Slaughter TF, Sreeram G, Sharma AD, El-Moalem H, East CJ, Greenberg CS (2001): Reversible shear-mediated platelet dysfunction during cardiac surgery as assessed by the PFA - 100 platelet function analyzer. Blood Coagul Fibrinolysis $\underline{12}, 85-93$

Song JH, Cronan JJ (1998): Percutaneous biopsy in diffuse renal disease: comparison of 18- and 14-gauge automated biopsy devices. J Vasc Interv Radiol $\underline{9}, 651-655$

Stiles KP, Hill C, LeBrun CJ, Reinmuth B, Yuan CM, Abbott KC (2001): The impact of bleeding times on major complication rates after percutaneous real - time ultrasound guided renal biopsies. J Nephrol 14, 275-279

Stratta P, Canavese C, Marengo M, Mesiano P, Besso L, Quaglia M, Bergamo D, Monga G, Mazzucco G, Ciccone G (2007): Risk management of renal biopsy: 1387 cases over 30 years in a single centre. Eur J Clin Invest $\underline{37}$, 954-963

Thompson BC, Kingdon E, Johnston M, Tibballs J, Watkinson A, Jarmulowicz M, Burns A, Sweny P, Wheeler DC (2004) : Transjugular kidney biopsy. Am J Kidney Dis $\underline{43}, 651-662$

Wahba A, Sander S, Birnbaum DE (1998): Are in - vitro platelet function tests useful in predicting blood loss following open heart surgery? Thorac Cardiovasc Surg $\underline{46}, 228-231$ 
Whittier WL, Korbet SM (2004 a): Renal biopsy: update. Curr Opin Nephrol Hypertens $\underline{13}, 661-665$

Whittier WL, Korbet SM (2004 b): Timing of complications in percutaneous renal biopsy. J Am Soc Nephrol 15, 142-147

Wilczek HE (1991): Percutaneous needle biopsy of the renal allograft. A clinical safety evaluation of 1129 biopsies. Transplantation $\underline{51}, 1140$

Wiseman DA, Hawkins R, Numerow LM, Taub KJ (1990): Percutaneous renal biopsy utilizing real time, ultrasonic guidance and a semiautomated biopsy device. Kidney Int $\underline{38}, 347-349$

Wuillemin WA, Gasser KM, Zeerleder SS, Lämmle B (2002): Evaluation of a Platelet Function Analyser (PFA - 100) in patients with a bleeding tendency. Swiss Med Wkly 132, 443-448 


\section{Danksagung}

Danken möchte ich Herrn Prof. Dr. Strutz für die Überlassung des Dissertationsthemas und für die stets engagierte und motivierende Betreuung. Des Weiteren möchte ich Herrn Dr. Jung von der Abteilung medizinische Statistik der Universität Göttingen danken für die stets engagierte Unterstützung und Hilfestellung bei der statistischen Auswertung meiner Dissertationsarbeit. 


\section{Curriculum Vitae}

Mein Name ist Sylvia Maria Wachendorfer. Ich wurde am 15. November 1984 als zweites Kind von Stefan Remigius Wachendorfer (Realschullehrer) und Wilma Maria Wachendorfer (Hausfrau) geboren.

Von 1991 bis 1995 besuchte ich die Lammerberg-Grundschule Tailfingen, von 1995 bis 2001 das Progymnasium Tailfingen und anschließend von 2001 bis 2004 das Gymnasium Ebingen, wo ich im Juni 2004 mein Abitur machte.

Im Wintersemester 2004/2005 begann ich mein Medizinstudium an der Georg August - Universität Göttingen. Im Sommersemester 2006 absolvierte ich erfolgreich die Erste Ärztliche Prüfung.

Am 17. August 2009 habe ich mein praktisches Jahr begonnen. 